\title{
Dual inhibitors of hepatitis $C$ virus and hepatocellular carcinoma: design, synthesis and docking studies
}

\author{
Mostafa MM El-Miligy ${ }^{*}{ }^{1}$, Samia M Rida ${ }^{1}$, Fawzia A Ashour ${ }^{1}$, Mona H Badr ${ }^{1}$, Ehab M \\ El-Bassiony1, Maha A El-Demellawy² \& Ashraf M Omar ${ }^{2}$ \\ ${ }^{1}$ Pharmaceutical Chemistry Department, Faculty of Pharmacy, Alexandria University, Alexandria 21521, Egypt \\ ${ }^{2}$ Medical Biotechnology Department, Genetic Engineering \& Biotechnology Research Institute, City for Scientific Research \& \\ Technology Applications, Alexandria, Egypt \\ * Author for correspondence: Tel.: +20 100170 8632; Fax: +20 3487 3273; mostafa.elmiligy@alexu.edu.eg
}

\begin{abstract}
Aim: Simultaneous inhibition of hepatitis $\mathrm{C}$ virus (HCV) and hepatocellular carcinoma (HCC) may enhance anti-HCV effects and reduce resistance and side effects. Results/methodology: Novel hybrid derivatives were designed and synthesized to exhibit dual activity against HCV and its associated major complication, HCC. The synthesized compounds were screened for their potential activity against HCV and HCC. Compounds $\mathbf{5 f}, \mathbf{5 j}, \mathbf{5 l}, \mathbf{5 p}, \mathbf{5 q}, \mathbf{5 r}, \mathbf{6 c}$ and $\mathbf{6 d}$ exhibited potential in vitro anticancer activity against HCC cell line HepG2, while compounds $\mathbf{5 a}, \mathbf{5 l}, \mathbf{5 p}$ and $\mathbf{5 v}$ showed in vitro anti-HCV activity. Docking studies suggested that the newly synthesized compounds could suppress HCC through VEGFR2 tyrosine kinase inhibition. Conclusion: Compounds $\mathbf{5 l}$ and $\mathbf{5 p}$ exhibited dual activity against $\mathrm{HCV}$ and $\mathrm{HCC}$ in vitro.
\end{abstract}

Lay abstract: There is a close relation between chronic hepatitis C virus (HCV) infection and development of hepatocellular carcinoma (HCC). Dual inhibition of HCV and HCC could enhance anti-HCV activity and reduce resistance of $\mathrm{HCC}$ to treatment. Novel compounds were designed and synthesized to exhibit dual activity against HCV and HCC. The synthesized compounds were screened for their potential in vitro activity against $\mathrm{HCV}$ and the $\mathrm{HCC} \mathrm{HepG} 2$ cell line. Compounds $5 \mathrm{I}$ and $\mathbf{5 p}$ exhibited dual activity against HCV and HCC in vitro. Further studies predicted that the target compounds could suppress HCC.

Graphical abstract

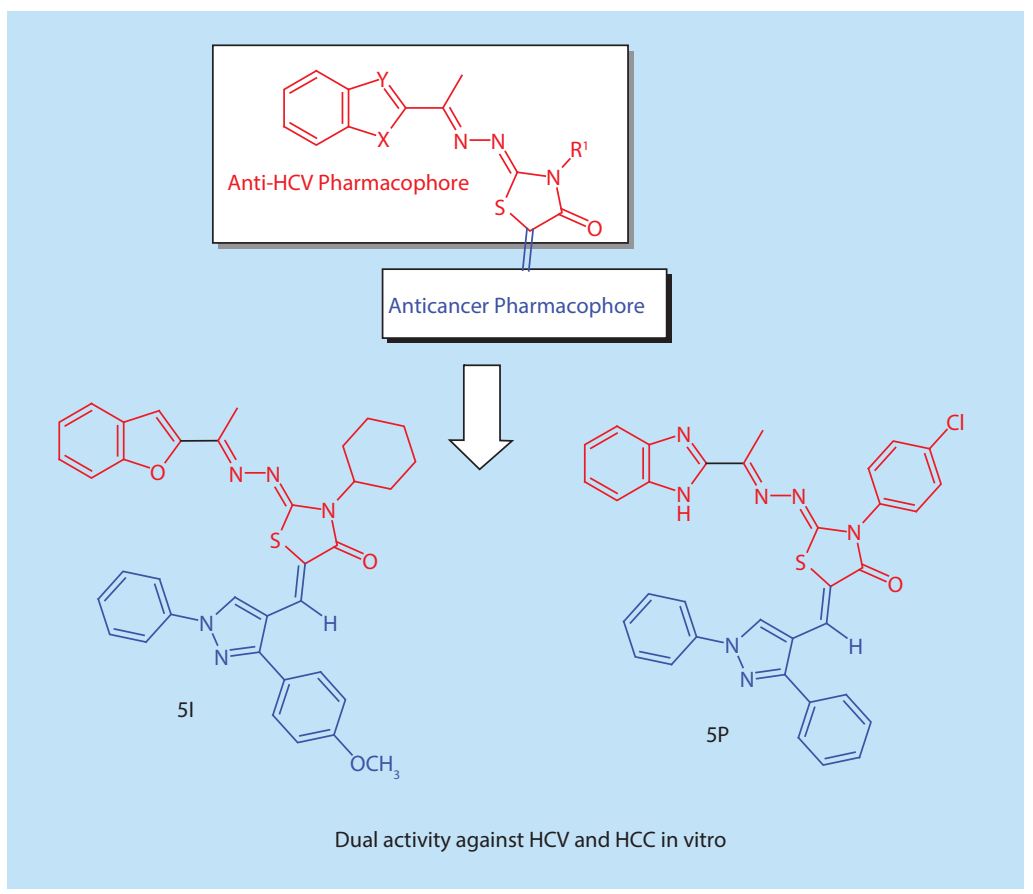


First draft submitted: 19 April 2017; Accepted for publication: 11 September 2017; Published online: 25 October 2017

Keywords: benzimidazole $\bullet$ benzofuran $\bullet$ docking $\bullet \mathrm{HCC}$ inhibitors $\bullet \mathrm{HCV}$ inhibitors $\bullet$ thiazolidinone

Hepatitis $\mathrm{C}$ virus $(\mathrm{HCV})$ is a contagious liver disease. Chronic HCV infection results in chronic inflammation that can lead to liver fibrosis, cirrhosis, hepatocellular carcinoma (HCC) and death [1]. About 71 million people are chronically infected and at risk of developing liver cirrhosis and/or HCC. Approximately 399,000 people die from hepatitis C-related liver diseases every year [2].

The standard treatment therapy for patients with chronic HCV infection consists of a combination of pegylated IFN- $\alpha$ injections and oral ribavirin for 24-48 weeks. However, this therapy suffers from many limitations including limited efficacy against some virus genotypes and severe side effects such as anemia.

The discovery of direct-acting antiviral agents led to development in the treatment of chronic HCV infection. The preferred treatment regimen depends on the viral genotype, if the patient has cirrhosis or not, on previous drug treatment and drug costs [3]. In 2011, the US FDA approved the new antiviral drugs boceprevir and telaprevir to be used with the standard therapy [4]. This new combination increased the incidence of anemia [4]. In 2013, the FDA approved simeprevir and sofosbuvir to be used in combination with interferon and ribavirin. However, this combination showed excellent activity only against some virus genotypes [4].

Worldwide, HCC is one of the top three cancer killers [5]. In 2012, 782,200 cases were diagnosed and were responsible for 746,000 deaths [6]. Chronic HCV increases risk of developing HCC 20-30-fold as compared with uninfected patients. Nearly $2.5 \%$ of chronic HCV patients develop HCC [7]. Many reports have illustrated the role of some viral proteins, particularly phosphorylated NS5A, in the development of HCC. The HCV protein NS5A is activated by human tyrosine kinase to produce the active phosphorylated form [8], which upregulates COX-2 expression and promotes the release of matrix metalloprotinase- 2 and 9 associated with tumor progression and recurrence in HCC patients [9,10]. In addition, phosphorylated NS5A interacts with and activates certain kinases involved in signal transduction pathways responsible for proliferation of HCC cells [10]. Moreover, phosphorylated NS5A inhibits apoptosis through inhibiting p53 function and inactivating the cell defense mechanism RNAdependent protein kinase [10-13]. Also, the highly vascular nature of HCC indicates the activation of angiogenic signaling pathways, activated mainly through receptor tyrosine kinases [14].

Currently, there is no effective therapy for HCC. Sorafenib is approved as first-line treatment but it has severe adverse side effects such as dermatological adverse effects [15], diarrhea [16] or arterial hypertension [17]. Regorafenib has recently been approved as second-line therapy for HCC after failure of sorafenib [18,19]. The search for more effective molecular agents than sorafenib or combinations of therapy might improve response and survival rates in HCC patients. Radioembolization [20] and immunotherapy [21] are still in clinical trials. Thus, there is an urgent need for new therapeutic agents for HCV infection and its associated major complication, HCC. A literature survey revealed that many derivatives containing benzofuran, its bioisostere benzimidazole or thiazolidinone I-VI were active against HCV (Figure 1) [22-27]. On the other hand, many compounds containing (2-oxoindolin-3-ylidene) moiety such as sunitinib, SU6668, NP603, NP506 and BIBF 1120 (VII-XI) showed anticancer activity against many tumors through inhibition of kinases (Figure 2) [14,28-31]. In addition, 1,3-diaryl-1H-pyrazole moiety is an interesting scaffold for the design of kinase inhibitors. Many compounds containing this moiety (XII-XIV) displayed kinase inhibitory activity and showed antiproliferative activity against different types of cancer [32-35]. It was found that 2-oxoindolin-3-ylidene scaffold in the multikinase inhibitor sunitinib is responsible for hydrogen bond interaction with the hinge region [36] while the pyrazole ring was reported to recognize the ATP-binding site [36] either by forming hydrogen bonds [37], arene-cation interactions or by contribution to the hydrophobic interactions [33,38]. Therefore, we can expect that the attachment of well-known kinase inhibitor pharmacophores such as substituted 2-oxoindolin-3-ylidene or 3-aryl-1-phenyl-1 $H$-pyrazole will enhance the kinase inhibitory and anticancer activity of our compounds to be active against HCC.

Consequently, we focused on the design and synthesis of novel hybrid compounds as dual anti-HCV and antiHCC. The target compounds were designed to hybridize an anticancer kinase inhibitor pharmacophore composed of 2-oxoindolin-3-ylidene or 1,3-diaryl-1 $H$-pyrazole to an anti-HCV pharmacophore composed of benzofuran or its bioisostere benzimidazole linked to thiazolidinone ring at position 2 through three atoms spacer. The substituents at position 3 of thiazolidinone moiety were chosen to include either aliphatic cyclohexyl group or aromatic unsubstituted phenyl or substituted with electron withdrawing $(\mathrm{Cl})$ or donating $\left(\mathrm{OCH}_{3}\right)$ groups. Besides, 


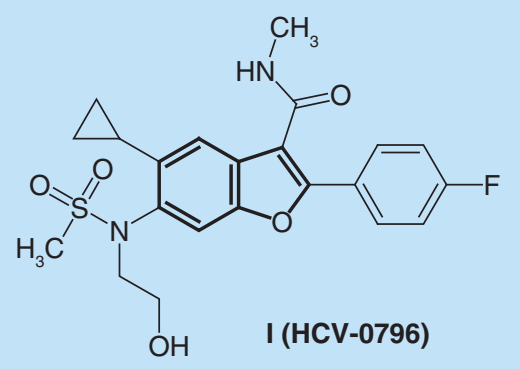

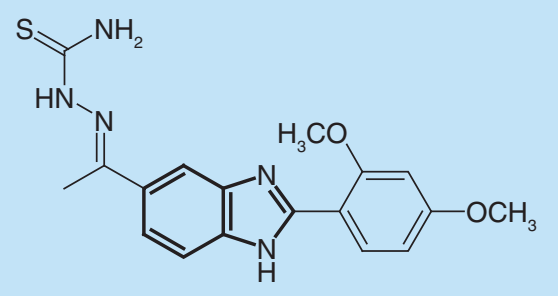

III

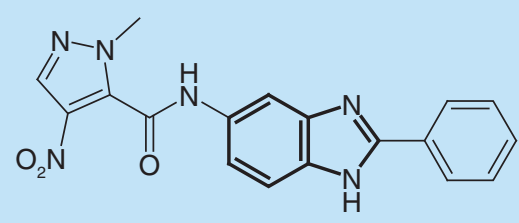

IV

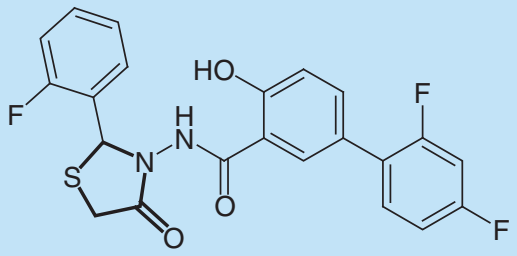

V

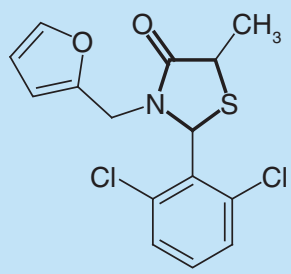

VI

Figure 1. Benzofuran, benzimidazole or thiazolidinone derivatives active against the hepatitis $C$ virus.

the phenyl moiety at position 3 of 1,3-diaryl- $1 H$-pyrazole pharmacophore was substituted with either electron withdrawing $(\mathrm{Cl})$ or donating $\left(\mathrm{OCH}_{3}\right)$ groups to change $\pi$-electron density of the target compounds and hence binding affinity and activity (Figures $3 \& 4$ ).

\section{Results \& discussion}

\section{Chemistry}

The target compounds were prepared (Figure 4) through the reaction of 2-acetylbenzofuran or 2-acetyl benzimidazole with substituted thiosemicarbazides in ethanol containing few drops of glacial acetic acid as a catalyst [39,40] to yield the corresponding thiosemicarbazones, which were then cyclized using chloroacetic acid and anhydrous sodium acetate in glacial acetic acid [39,41] to give the key intermediates, 4-thiazolidinones. Infrared (IR) spectra of thiosemicarbazones intermediates (2a-i) were characterized by the disappearance of absorption band due to $\mathrm{C}=\mathrm{O}$ at $1678-1674 \mathrm{~cm}^{-1}$ and the appearance of N-C $=$ S amide I, II, III and IV bands at 1571-1560, 1304-1300, 1187-1180, 927-921. Their ${ }^{1} \mathrm{H}-\mathrm{NMR}$ spectra showed a singlet at 2.34-2.53 p.p.m. corresponding to $-\mathrm{N}=\mathrm{C}-\mathrm{CH}_{3}$ in addition to two deuterium exchangeable singlets of $\mathrm{H}-\mathrm{N}-(\mathrm{C}=\mathrm{S})-\mathrm{N}-\mathrm{H}$ at $7.99-10.43$ and $10.43-11.02$ p.p.m. The other signals appeared at their expected chemical shifts. IR spectra of 4 -thiazolidinone intermediates (3a-i) were characterized by the disappearance of $\mathrm{N}-\mathrm{C}=\mathrm{S}$ amide I, II, III and IV bands and appearance of $\mathrm{C}=\mathrm{O}$ band at $1726-1714 \mathrm{~cm}^{-1}$. Their ${ }^{1} \mathrm{H}-\mathrm{NMR}$ spectra were characterized by the disappearance of $\mathrm{H}-\mathrm{N}-(\mathrm{C}=\mathrm{S})-\mathrm{N}-\mathrm{H}$ signals at 7.99-11.02 p.p.m. and the appearance of a singlet at 3.94-4.17 p.p.m. attributed to thiazolidinone $\mathrm{C}^{5}-\mathrm{H}_{2}$. The other protons appeared at their expected chemical shifts. 4-thiazolidinones were subjected to Knoevenagel condensation with either 3-aryl-1-phenyl-pyrazole-4-carbaldehydes (4a-c) or isatin using piperidine in dry dioxane [39] to give the targeted compounds $\mathbf{5} \mathbf{a}-\mathbf{x}$ and $\mathbf{6 a}-\mathbf{h}$ respectively. The IR spectra of compounds $\mathbf{5 a - x}$ retained the characteristic absorption band of $\mathrm{C}=\mathrm{O}$ of thiazolidinone at $1719-1685 \mathrm{~cm}^{-1}$, while their ${ }^{1} \mathrm{H}-\mathrm{NMR}$ spectra were characterized by the disappearance of thiazolidinone $\mathrm{C}^{5}-\mathrm{H}_{2}$ singlet at 3.94-4.17 p.p.m. and the appearance of two singlets at 7.51-7.91 and 8.77-8.99 p.p.m. assigned for vinylic $-\mathrm{C}=\mathrm{CH}$ and pyrazole $\mathrm{C}^{5}-\underline{\mathrm{H}}$, respectively. The other protons appeared at their expected chemical shifts. The IR of compounds $\mathbf{6 a}-\mathbf{h}$ revealed an additional $\mathrm{C}=\mathrm{O}$ band at 1687-1663 $\mathrm{cm}^{-1}$ assigned for amide I band of 2-oxoindolin together with $\mathrm{C}=\mathrm{O}$ of thiazolidinone at 1705$1692 \mathrm{~cm}^{-1}$. Another absorption band appeared at 3193-3119 cm-1 corresponding to the $\mathrm{N}-\mathrm{H}$ of 2-oxoindolin. In addition, their ${ }^{1} \mathrm{H}-\mathrm{NMR}$ spectra were characterized by the disappearance of thiazolidinone $\mathrm{C}^{5}-\mathrm{H}_{2}$ singlet at 
<smiles>CCN(CC)CCNC(=O)c1c(C)[nH]c(/C=C2\C(=O)Nc3ccc(F)cc32)c1C</smiles>

VII (Sunitinib)<smiles>Cc1[nH]c(/C=C2\C(=O)Nc3ccccc32)c(C)c1CCC(=O)O</smiles>

VIII (SU6668)<smiles>COc1cc(OC)cc(-c2ccc3c(c2)NC(=O)/C3=C\c2[nH]c(C)c(CCC(=O)O)c2C)c1</smiles>

IX (NP603)<smiles>Cc1[nH]c(/C=C2\C(=O)Nc3ccc(C(=O)NNc4ccccc4)cc32)c(C)c1CCC(=O)O</smiles>

$X(N P 506)$<smiles>COC(=O)c1ccc2c(c1)NC(=O)/C2=C(/Nc1ccc(N(C)C(=O)CN2CCN(C)CC2)cc1)c1ccccc1</smiles>

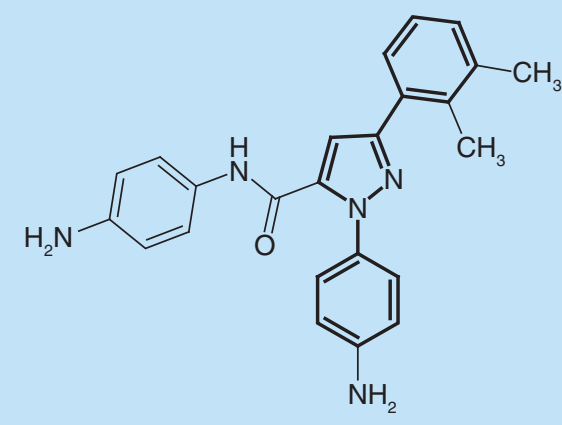

XII

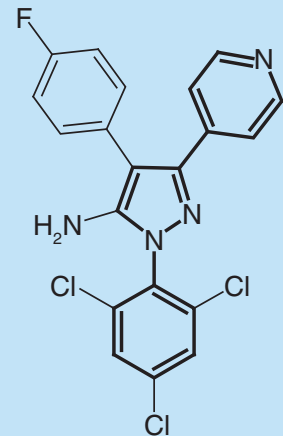

XIII

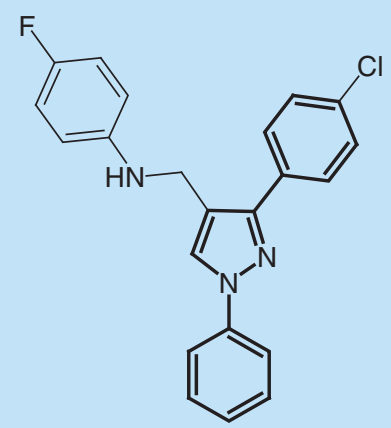

XIV

Figure 2. Some anticancer kinase inhibitors containing 2-oxoindolin-3-ylidene or 1,3-diaryl-1H-pyrazole moieties.

3.94-4.17 p.p.m. and appearance of deuterium exchangeable singlet at $11.15-11.31$ p.p.m. corresponding to N-H of 2-oxoindolin.

During the present investigation, we tried first to obtain our target compounds $\mathbf{5 a - x}$ through condensation of 3-(4-substitutedphenyl)-1-phenyl-1 $H$-pyrazole-4-carbaldehydes (4a-c) with the active methylene of thiazolidinone ring of compounds (3a-i) in acetic acid in the presence of anhydrous sodium acetate according to the previously reported reaction conditions used for the synthesis of analogous compounds [42-44]. Unfortunately, one and the same unexpected product (7) was obtained rather than the expected condensed (Figure 4). The IR spectrum of the unexpected product from the condensation of the benzofuran thiazolidinone derivative (3d) with the pyrazole aldehyde (4b) under the same conditions lacked the C-O-C bands of benzofuran and showed the bands for $\mathrm{C}=\mathrm{O}$ at $1699 \mathrm{~cm}^{-1}$ and $\mathrm{C}=\mathrm{N}$ at $1619 \mathrm{~cm}^{-1}$, while the IR spectrum of the unexpected product from the condensation 


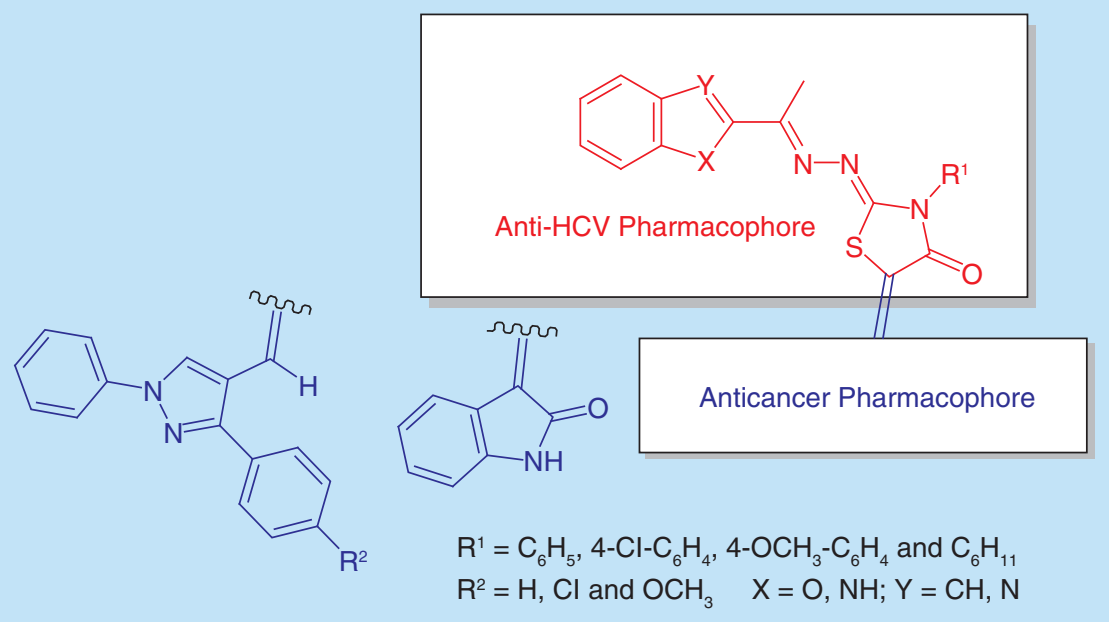

Figure 3. Design of dual anti-hepatitis $\mathrm{C}$ virus and anticancer hybrids.

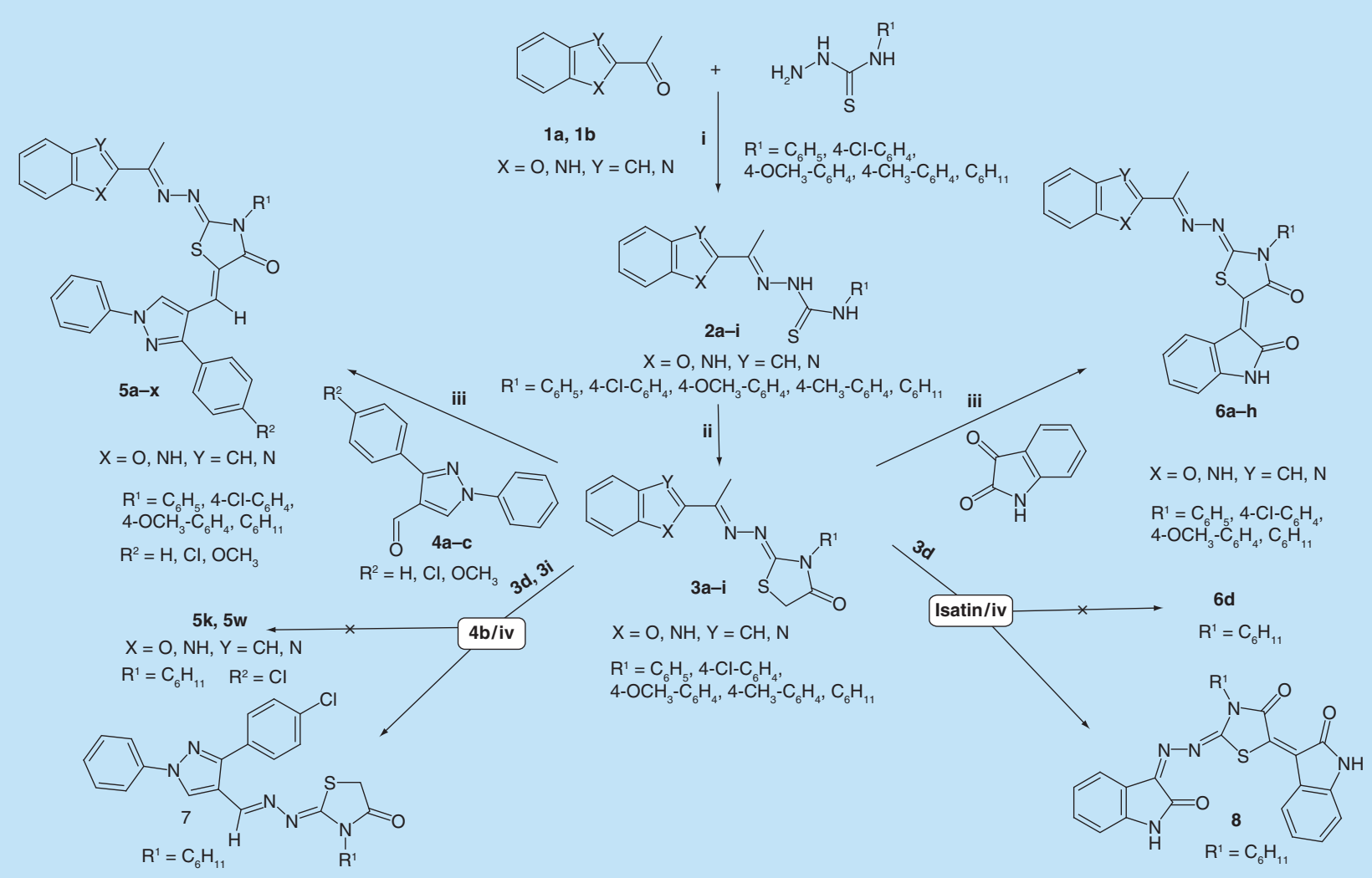

Figure 4. Synthesis of the target dual anti-hepatitis $\mathrm{C}$ virus and anticancer hybrids (5a-x) and (6a-h). Reagents: (i) Ethanol; (ii) $\mathrm{ClCH} 2 \mathrm{COOH}, \mathrm{CH} 3 \mathrm{COOH}, \mathrm{CH} 3 \mathrm{COONa}$; (iii) Piperdine, Dry Dioxane; (iv) $\mathrm{CH} 3 \mathrm{COOH}, \mathrm{CH} 3 \mathrm{COONa}$.

of the benzimidazole thiazolidinone derivative (3i) with the pyrazole aldehyde (4b) also lacked the N-H band of benzimidazole at 3333-3241 $\mathrm{cm}^{-1}$ and showed the bands for $\mathrm{C}=\mathrm{O}$ at $1694 \mathrm{~cm}^{-1}$ and $\mathrm{C}=\mathrm{N}$ at $1618 \mathrm{~cm}^{-1}$. Comparing the two unexpected products, both were found to have the same melting point, the same $\mathrm{R}_{\mathrm{f}}$ value in thin layer chromatography and the same elemental analysis. Moreover, the ${ }^{1} \mathrm{H}-\mathrm{NMR}$ spectra of the unexpected 
products obtained from the benzimidazole or benzofuran derivatives lacked the aromatic protons of benzimidazole or benzofuran, the singlet corresponding to $-\mathrm{N}=\mathrm{C}-\mathrm{CH}_{3}$ at 2.23-2.60 p.p.m. and the deuterium exchangeable singlet corresponding to N-H of benzimidazole at $11.22-12.56$ p.p.m. In addition, the unexpected compounds retained the singlet at 3.94-4.14 p.p.m. attributed to thiazolidinone $\mathrm{C}^{5}-\mathrm{H}_{2}$ and both were also characterized by the appearance of two singlets at $8.30-8.50$ and $8.80-8.95$ p.p.m. assigned for $-\mathrm{CH}=\mathrm{N}-\mathrm{N}=$ and pyrazole $\mathrm{C}^{5}-\underline{\mathrm{H}}$, respectively. Furthermore, the ${ }^{1} \mathrm{H}-\mathrm{NMR}$ spectra of the two unexpected products obtained from the benzimidazole and benzofuran derivatives were superimposed and stacked indicating they were one and the same compound (Supplementary Figure 1). We assumed that acetic acid resulted in hydrolysis of the hydrazono linkage followed by acid catalyzed condensation of the produced 2-hydrazono-3-cyclohexyl-thiazolidin-4-one with the pyrazole aldehyde (aldehydes are more reactive than ketones toward nucleophiles; Supplementary Figure 2). In addition, during our trials to perform the condensation of thiazolidinone derivatives (3a-i) with isatin in acetic acid in the presence of sodium acetate, the benzofuran thiazolidinone (3d) was reacted with isatin and the unexpected product (8) was obtained in 38\% yield (Figure 4). The IR spectrum of the unexpected product (8) lacked the C-O-C bands of benzofuran while showing the bands of $\mathrm{C}=\mathrm{O}$ at $1725 \mathrm{~cm}^{-1}$ and $\mathrm{C}=\mathrm{N}$ at $1608 \mathrm{~cm}^{-1}$. The ${ }^{1} \mathrm{H}-\mathrm{NMR}$ spectrum of compound (8) lacked the singlet corresponding to $-\mathrm{N}=\mathrm{C}-\mathrm{CH}_{3}$ at 2.24-2.52 p.p.m. and the singlet corresponding to thiazolidinone $\mathrm{C}^{5}-\mathrm{H}_{2}$ at 3.94-4.14 p.p.m.. The spectrum also showed only eight aromatic protons instead of nine in the expected condensed product. In addition, two deuterium exchangeable singlets appeared at 10.79 and 11.18 p.p.m. instead of one at 11.15-11.24 p.p.m. in the expected condensed product. However, on using two moles of isatin (instead of 1.1 moles) the yield increased to $63 \%$. This was a further confirmation of our conclusion.

\section{Biological evaluation \\ In vitro hemolytic assay}

Compounds $\mathbf{5 a - x}$ and $\mathbf{6 a}-\mathbf{h}$ were subjected to the in vitro hemolytic assay to identify those which cause hemolysis to red blood cells (RBCs). Consequently, these compounds would be toxic in vivo so they were excluded from further biological screening. However, compounds $\mathbf{5 h}, \mathbf{5 s}, \mathbf{5 t}$ and $\mathbf{5 u}$ could not be dissolved in dimethylformamide (DMF) or DMSO under experimental conditions; therefore, these compounds were excluded from biological screening. It was found that compounds $5 \mathbf{a}-\mathbf{g}, 5 \mathbf{i}-1,5 \mathbf{p}-\mathbf{r}, 5 \mathrm{v}-\mathbf{w}$ and $\mathbf{6 b}-\mathbf{g}$ were nontoxic to RBCs, but compounds $\mathbf{5 m - 0}, \mathbf{5 x}, \mathbf{6 a}$ and $\mathbf{6 h}$ were found to cause hemolysis to RBCs, and were excluded from further testing (Supplementary Table 1).

\section{In vitro anticancer screening}

The safe compounds on RBCs (5a-g, 5i-1, 5p-r, 5v-w, 6b-g) were screened for their potential anticancer activity against human HCC cell line HepG2. HepG2 cells were exposed to the test compounds, then viability of cells was measured using neutral red uptake assay as described by Borenfreund and Puerner [45]. This assay depends on the fact that neutral red dye can be incorporated into the lysosomes of living cells [46] providing a quantitative assay to the cytotoxic effects. The results were interpreted to calculate both the concentration causing $50 \%$ cancer cell death $\left(\mathrm{IC}_{50}\right)$ of each compound and the maximum safe concentration that cause $100 \%$ viability $\left(\mathrm{LD}_{0}\right.$; to be used in the in vitro anti-HCV testing) using GraphPad InStat 3.0 software (Figure 5 \& Supplementary Table 2) [47].

It was reported that $\mathrm{IC}_{50}$ values $<100 \mu \mathrm{g} / \mathrm{ml}$ reflected a potential anticancer activity, while values between 100 and $1000 \mu \mathrm{g} / \mathrm{ml}$ indicated a moderate anticancer activity [48]. The results revealed that eight compounds showed potential in vitro anticancer activity against $\mathrm{HepG} 2$ cells with $\mathrm{IC}_{50}$ values $<100 \mu \mathrm{g} / \mathrm{ml}$. Compounds $\mathbf{5 p}$, 5q, 5r and $\mathbf{6 d}$ exhibited the most potent anticancer activity with $\mathrm{IC}_{50}$ values $<50 \mu \mathrm{g} / \mathrm{ml}$, while compounds $\mathbf{5 f}, \mathbf{5 j}$, $\mathbf{5 1}$ and 6c showed anticancer activity with $\mathrm{IC}_{50}$ values between 50 and $100 \mu \mathrm{g} / \mathrm{ml}$. The rest of our compounds showed a moderate anticancer activity with $\mathrm{IC}_{50}$ values between 100 and $1000 \mu \mathrm{g} / \mathrm{ml}$, except for compounds $\mathbf{5 d}$ and $\mathbf{5 w}$ which showed a very weak anticancer activity against HepG2 cells.

\section{In vitro cytotoxicity assay}

The safe compounds on RBCs (5a-g, 5i-1, 5p-r, 5v-w, 6b-g) were subjected to the in vitro cytotoxicity assay on human peripheral blood mononuclear cells (PBMCs). Viability of cells was measured using neutral red uptake assay as described by Borenfreund and Puerner [45] to determine the concentrations of each compound that were not cytotoxic. The results were interpreted to calculate both the lethal concentration that kills $50 \%$ of cells $\left(\mathrm{LD}_{50}\right)$ and the maximum safe concentration that causes $100 \%$ viability $\left(\mathrm{LD}_{0}\right)$ of each compound using GraphPad InStat 3.0 software (Figure $6 \&$ Supplementary Table 3) [47]. 


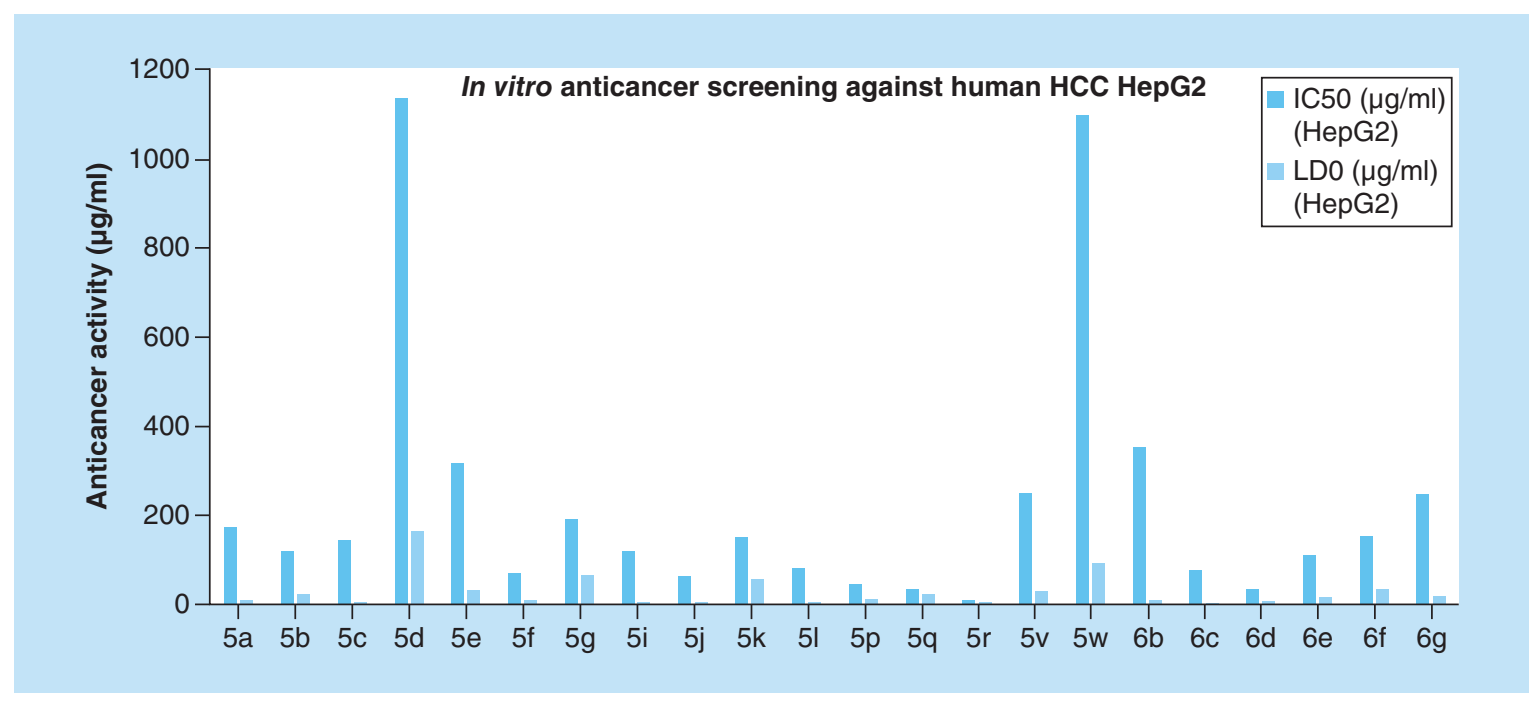

Figure 5. In vitro anticancer screening $\left(\mathrm{IC}_{50}\right.$ and $\left.\mathrm{LD}_{0}\right)$ against human hepatocellular carcinoma cell line HepG2. HCC: Hepatocellular carcinoma.

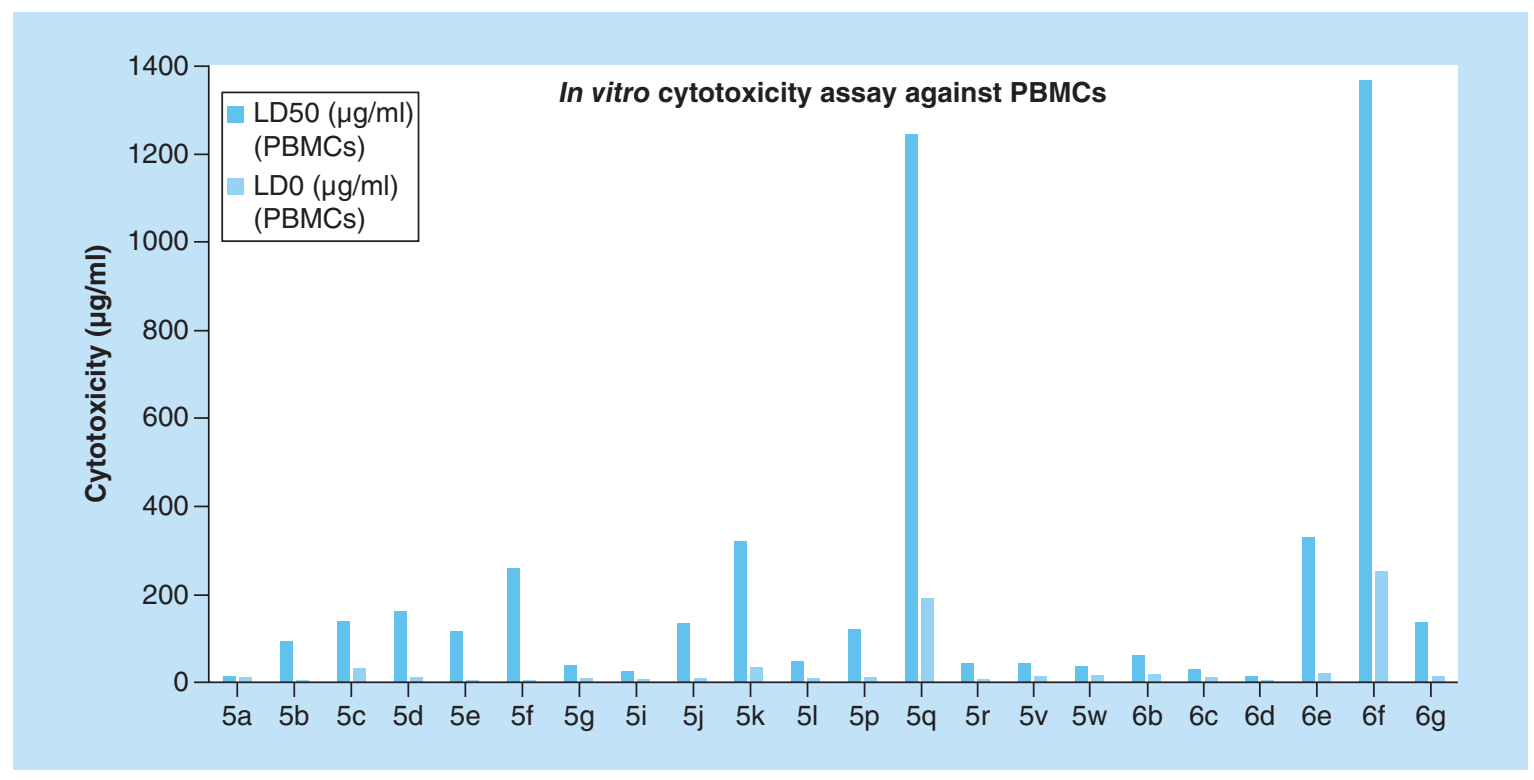

Figure 6. In vitro cytotoxicity $\left(\mathrm{LD}_{50}\right.$ and $\left.\mathrm{LD}_{0}\right)$ assay against peripheral blood mononuclear cells. PBMCs: Peripheral blood mononuclear cells.

The above results showed that the concentrations that retained $100 \%$ viability of the cells $\left(\mathrm{LD}_{0}\right)$ ranged from 4.26 to $33.29 \mu \mathrm{g} / \mathrm{ml}$. Exceptions were compounds $\mathbf{5 q}$ and $\mathbf{6 f}$ that showed relatively high $\mathrm{LD}_{0}$ values.

One of the most important criteria of an anticancer agent is its ability to discriminate between cancer and normal cells. To assess the selectivity of the active newly synthesized compounds, selectivity index was calculated for the compounds which showed potential in vitro anticancer effect against HepG2 cells. Selectivity index is a measure of the selectivity of the drug candidate toward cancer cells rather than normal cells (selectivity index $=\mathrm{LD}_{50}$ on normal cells $/ \mathrm{IC}_{50}$ on cancer cells). It was also reported that compounds with selectivity index values larger than three could be considered as highly selective (Table 1) [48].

Compounds 5f, 5q and $\mathbf{5 r}$ showed selectivity index values larger than three, and therefore could be considered selective against HepG2 cells. Interestingly, compound $\mathbf{5 q}$ could be considered as a promising anticancer lead compound as it showed a remarkable anticancer effect on HepG2 cancer cells $\left(\mathrm{IC}_{50}=35.72 \mu \mathrm{g} / \mathrm{ml}\right)$, and a safer 
Table 1. Selectivity index ${ }^{\dagger}$ values for the active in vitro anticancer compounds.

\begin{tabular}{|c|c|c|c|}
\hline Compound ID & $\mathrm{LD}_{50}$ (PBMCs) $(\mu \mathrm{g} / \mathrm{ml})$ & ${ }^{\prime} C_{50}(\mathrm{HepG})(\mu \mathrm{g} / \mathrm{ml})$ & Selectivity index \\
\hline $5 f$ & 259.71 & 69.44 & 3.74 \\
\hline $5 \mathbf{j}$ & 134.00 & 63.81 & 2.10 \\
\hline 51 & 46.89 & 82.08 & 0.57 \\
\hline $5 p$ & 120.46 & 46.51 & 2.58 \\
\hline $5 q$ & 1246.96 & 35.72 & 62.90 \\
\hline $5 r$ & 44.00 & 11.01 & 3.99 \\
\hline $6 d$ & 14.32 & 34.28 & 0.42 \\
\hline
\end{tabular}

effect on PBMCs normal cells $\left(\mathrm{LD}_{0}=190.45 \mu \mathrm{g} / \mathrm{ml}\right)$. Moreover, the concentration that killed $100 \%$ of cancer cells $\left(\mathrm{IC}_{100}\right)$ was calculated for this compound (using GraphPad InStat 3.0 software) [47], and was found to be $94.54 \mu \mathrm{g} / \mathrm{ml}$. This value was also much lower than the $\mathrm{LD}_{0}$ of this compound on PBMCs. The selectivity index of this compound was found to be 62.9. This very high value indicated that compound $\mathbf{5 q}$ was highly selective toward HCC HepG2 cells.

\section{In vitro anti-HCV screening}

HCC HepG2 cell line was found to be the most susceptible cell culture system to HCV infection [49], therefore it could be used to support reliable and efficient progression of HCV. The safe compounds on RBCs (5a-g, 5i-1, 5p-r, 5v-w, 6b-g) were screened for their in vitro anti-HCV activity using the HCC HepG2 cell line infected with the HCV. Monitoring of the HCV viremia pre- and post-antiviral therapy through the detection of viral RNA using qualitative reverse transcription-PCR (RT-PCR) was adopted in the present investigation. This technique was reported to be the most frequently used, reliable and sensitive technique [50]. Inhibition of viral replication was detected by amplification of viral RNA segments by the RT-PCR technique both in the cultivated infected cells alone (as a positive control) and at the specified dose for each test compound at optimal temperature. The test compound was considered to be active when it can inhibit the viral replication inside the HCV-infected HepG2 cells, as evidenced by the disappearance of the viral RNA-amplified products detected by the RT-PCR (compared with positive control).

The used concentration of each compound was chosen to be lower than the $\mathrm{LD}_{0}$ on HepG2 cells. Therefore, $100 \%$ viability of HepG2 cells was maintained, to make sure that any inhibitory effect was due to anti-HCV activity rather than cytotoxic activity on HepG2 cells. Moreover, the used concentrations were also chosen to be lower than the $\mathrm{LD}_{0}$ on PBMCs. Consequently, these doses could be used for inhibition of HCV in normal cells infected with the virus. The results of the in vitro anti-HCV screening were listed in Table 2.

The results revealed that four compounds $\mathbf{5 a}, \mathbf{5 1}, \mathbf{5} \mathbf{p}$ and $\mathbf{5 v}$ exhibited in vitro anti-HCV activity, as indicated by the disappearance of the band corresponding to the fragment of 174 base pairs length (Figure 7 ). These compounds inhibited the virus replication at concentrations ranging from 5.10 to $13.80 \mu \mathrm{g} / \mathrm{ml}$.

\section{Structure-activity relationship}

Regarding the in vitro anticancer activity, it could be noticed that:

- Benzofuran derivatives containing aliphatic cyclohexyl ring as $\mathrm{R}^{1}$ appeared to be more active than their corresponding benzimidazoles, while benzimidazole derivatives containing $\mathrm{p}-\mathrm{Cl}$-phenyl ring as $\mathrm{R}^{1}$ were more active than their corresponding benzofurans. However, no general relation was observed to link different $\mathrm{R}^{1}$ substituents to the in vitro anticancer activity. This observation might support our rationale that the thiazolidinone nucleus was not a part of the anticancer pharmacophore.

- Some derivatives containing either 1,3-diaryl-1H-pyrazole or 2-oxoindolin-3-ylidene pharmacophores exhibited potential in vitro anticancer activity. It was observed that the benzofuran derivatives substituted by 2 oxoindolin-3-ylidene were more potent than their corresponding 1,3-diaryl-1H-pyrazole analogs. Nevertheless, the benzimidazole derivatives substituted by 2-oxoindolin-3-ylidene were found to be less potent than their 1,3-diaryl-1 $H$-pyrazole analogs. 


\begin{tabular}{|c|c|c|c|c|}
\hline Compound ID & $\left(\mathrm{LD}_{0}\right)$ PBMCs $(\mu \mathrm{g} / \mathrm{ml}$ & $\left(\mathrm{LD}_{0}\right) \mathrm{HepG} 2(\mu \mathrm{g} / \mathrm{ml})$ & Used dose $(\mu \mathrm{g} / \mathrm{ml})$ & Result \\
\hline $5 a$ & 11.17 & 10.15 & 10.10 & Positive \\
\hline $5 c$ & 32.45 & 5.88 & 5.80 & Negative \\
\hline $5 d$ & 11.43 & 163.55 & 11.40 & Negative \\
\hline $5 e$ & 4.26 & 31.12 & 4.30 & Negative \\
\hline $5 g$ & 8.88 & 66.72 & 8.80 & Negative \\
\hline $5 i$ & 7.60 & 0.05 & 0.05 & Negative \\
\hline $5 j$ & 9.90 & 5.08 & 5.00 & Negative \\
\hline $5 k$ & 33.29 & 56.74 & 20.00 & Negative \\
\hline 51 & 8.33 & 5.11 & 5.10 & Positive \\
\hline $5 v$ & 13.85 & 30.89 & 13.80 & Positive \\
\hline $5 w$ & 16.03 & 92.40 & 16.00 & Negative \\
\hline $6 b$ & 18.18 & 11.07 & 11.00 & Negative \\
\hline $6 c$ & 11.41 & 3.40 & 3.40 & Negative \\
\hline $6 d$ & 5.21 & 7.31 & 5.20 & Negative \\
\hline $6 e$ & 19.22 & 16.75 & 16.70 & Negative \\
\hline $6 f$ & 253.34 & 35.33 & 20.00 & Negative \\
\hline $6 \mathrm{~g}$ & 14.41 & 18.62 & 14.40 & Negative \\
\hline
\end{tabular}

PBMC: Peripheral blood mononuclear cell.

- The substituent $\mathrm{R}^{2}$ appeared to influence the anticancer activity of 1,3 -diaryl- $1 H$-pyrazole derivatives, as the methoxy derivatives were more potent than the chloro derivatives than the unsubstituted ones.

- The combination of methoxy group as $\mathrm{R}^{2}$ and $\mathrm{p}-\mathrm{Cl}$-phenyl as $\mathrm{R}^{1}$ generally increased the anticancer activity.

Regarding the in vitro anti-HCV activity, some relations were concluded linking structure to in vitro anti-HCV activity.

- The fact that all the anti-HCV compounds had a lipophilic substituent as $\mathrm{R}^{1}$, either aliphatic cyclohexyl group (51 and 5v) or aromatic phenyl and p-Cl-phenyl group (5a and $5 \mathbf{p}$ ), indicated that lipophilicity was an important structure feature regarding HCV inhibition.

- Both benzofuran and benzimidazole derivatives were found to inhibit the virus replication. This confirmed our hypothesis that both scaffolds have aided in the anti-HCV activity.

- An interesting observation was that no compounds containing (2-oxoindolin-3-ylidene) scaffold showed anti$\mathrm{HCV}$ activity, and all the active compounds were 1,3-diaryl- $1 H$-pyrazole derivatives. This suggested that 1,3diaryl- $1 H$-pyrazole scaffold played a certain role in $\mathrm{HCV}$ inhibition besides its role in HCC suppression.

- The substituent $\mathrm{R}^{2}$ appeared to influence the anti-HCV activity of 1,3-diaryl- $1 H$-pyrazole derivatives, as unsubstituted and methoxy derivatives were the active compounds.

\section{Docking studies inside VEGFR-2 active site}

The molecular modeling studies were performed using the Molecular Operating Environment [51] software [52]. The 3D structures and conformations of the enzymes were acquired from the Protein Data Bank (PDB) website [53]. The targeted compounds were docked into the active site of the kinase domain of the VEGFR-2 (PDB ID: 4AGD) to predict their potential kinase inhibition activity. The multikinase inhibitor sunitinib (VII) was the co-crystallized ligand, and was also utilized as the reference active drug (Supplementary Table 4) $[14,30]$. It was found that compounds (5a-x) and (6a-h) afforded docking scores higher than sunitinib (docking score $=-7.14 \mathrm{kcal} / \mathrm{mol}$ ), in most cases, when docked into the active site of the kinase domain of VEGFR-2. The mode of binding of the 


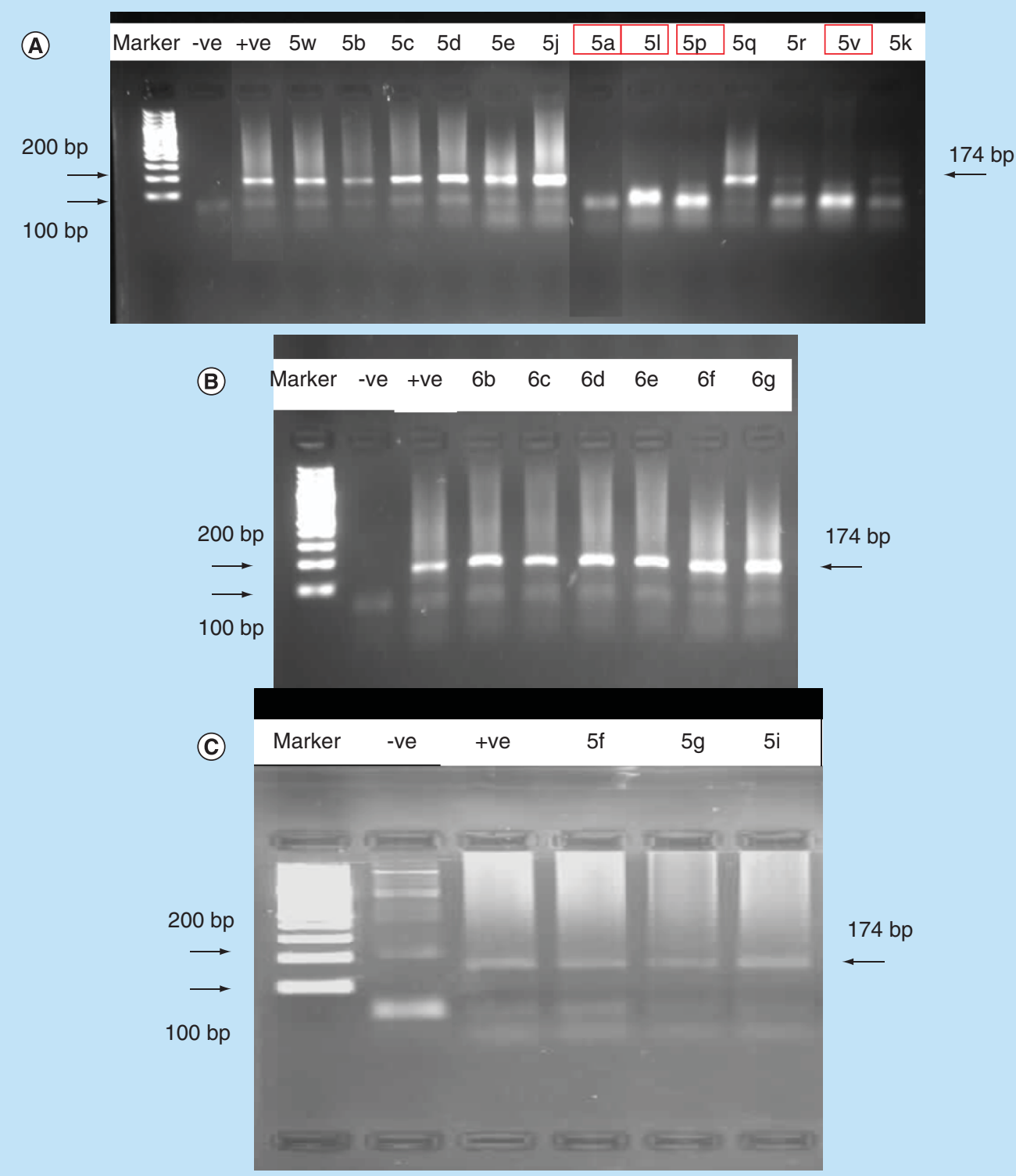

Figure 7. PCR amplification picture of hepatitis C virus RNA strand in the presence of 13 test compounds. The first band contains the molecular weight marker, while second and third bands are corresponding to negative and positive controls, respectively. The other bands show the effect of compounds (A) 5w, 5b, 5c, 5d, 5e, 5j, 5a, 5l, 5p, 5q, 5r, 5v and $5 \mathrm{k}$; (B) $6 \mathrm{~b}, 6 \mathrm{c}, 6 \mathrm{~d}, 6 \mathrm{e}, 6 \mathrm{f}$ and $6 \mathrm{~g}$; (C) $5 \mathrm{f}, 5 \mathrm{~g}$ and $5 \mathrm{i}$, respectively on hepatitis $\mathrm{C}$ virus RNA strand.

most promising in vitro anticancer compounds was studied with respect to the type of interactions with the receptor. Docking studies revealed that most of the newly synthesized compounds that exhibited promising in vitro anticancer activity mainly interacted with the receptor through the 1,3-diaryl- $1 H$-pyrazole $(\mathbf{5 p}$-rr, Figures $8-10$ ) or 2-oxoindolin-3-ylidene (6d, Figure 11) scaffolds. 1,3-Diaryl- $1 H$-pyrazole moiety appeared to contribute to the hydrophobic interactions with Phe 1047, Leu 840, Ala 866, Val 848 and Leu 1035 residues, as well as arene-cation interactions. In addition, 2-oxoindolin-3-ylidene moiety was responsible mainly for hydrogen bond interaction with Ser 930 residue. It was previously mentioned in our research objectives that 2-oxoindolin-3-ylidene scaffold in the type I inhibitor sunitinib (VII) was responsible for hydrogen bond interaction with the hinge region [36], while the pyrazole ring was reported to recognize the ATP-binding site [36] either by forming hydrogen bonds [37], arenecation interactions or by contribution to the hydrophobic interactions [33,38]. Therefore, docking studies supported our hypothesis that the attachment of well-known kinase inhibitor pharmacophores such as 2-oxoindolin-3-ylidene 


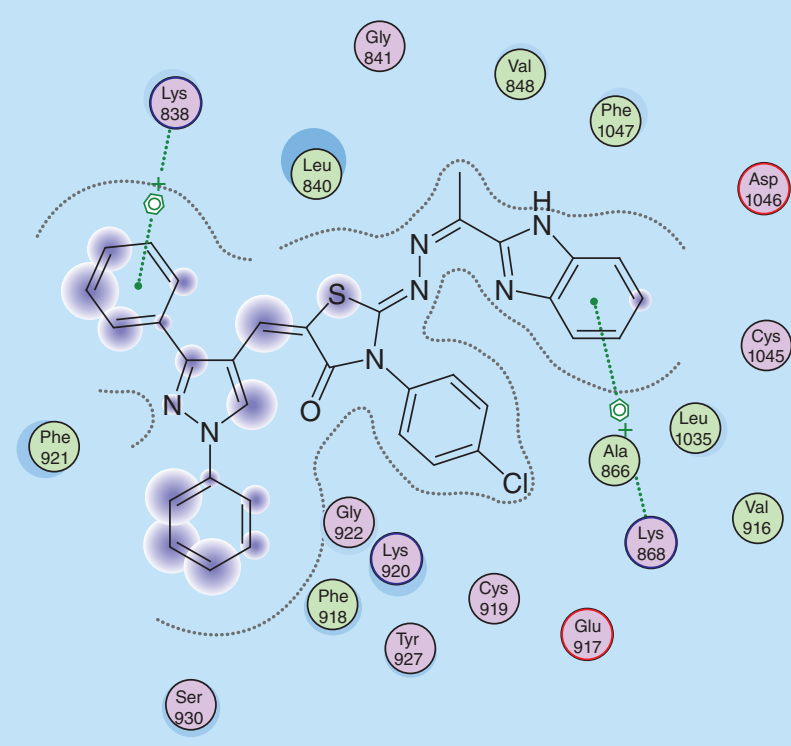

2D ligand-enzyme interaction

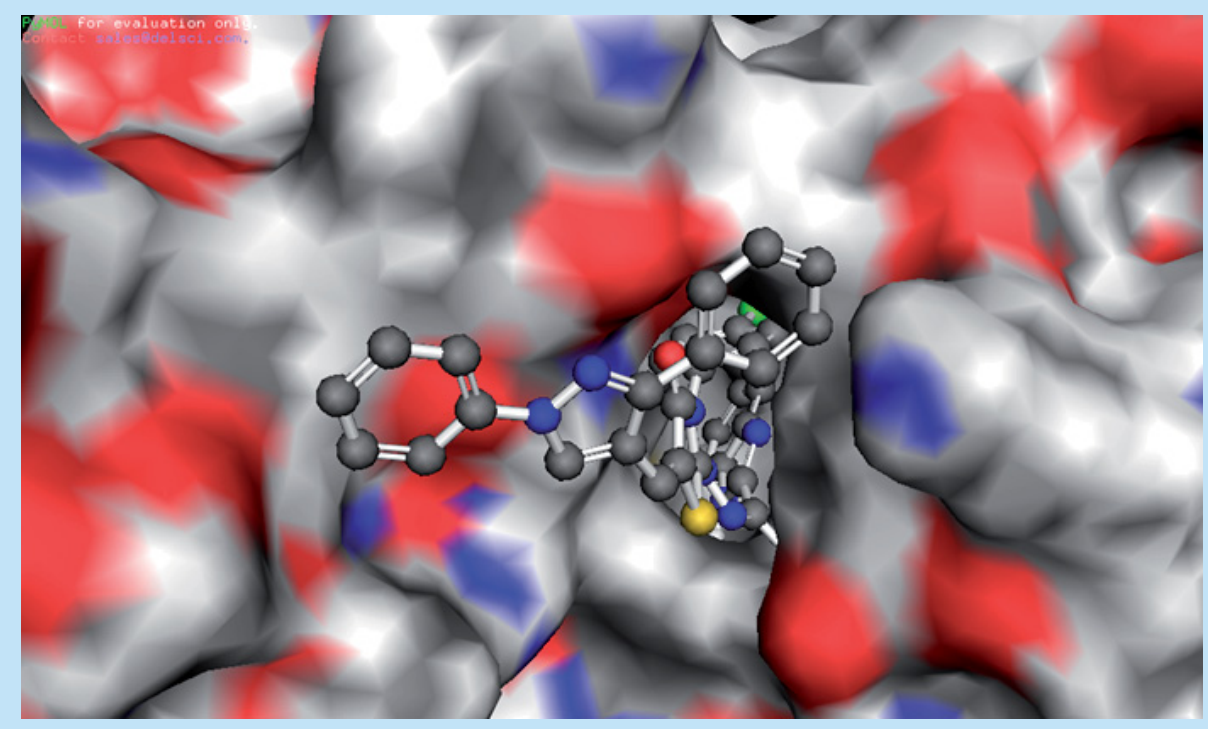

3D ligand-enzyme interaction

Figure 8. Mode of binding of compound $5 \mathrm{p}\left(\mathrm{IC}_{50}=46.51 \mu \mathrm{g} / \mathrm{ml}\right.$ and docking score $=-7.67 \mathrm{kcal} / \mathrm{mol}$, respectively $)$ inside VEGFR-2 active site.

or 3-aryl-1-phenyl-1H-pyrazole might enhance the kinase inhibitory and anticancer activity of our compounds so as to be active against HCC.

\section{Conclusion}

The present study was initiated aiming to design and synthesize novel compounds that exhibit dual activity against $\mathrm{HCV}$ and its associated major complication, $\mathrm{HCC}$ as an alternative to multidrug therapy. In order to achieve this target, hybrid compounds were designed to incorporate both anti-HCV and anticancer pharmacophores. The antiHCV pharmacophore was designed to encompass different chemical scaffolds such as benzofuran, benzimidazole 


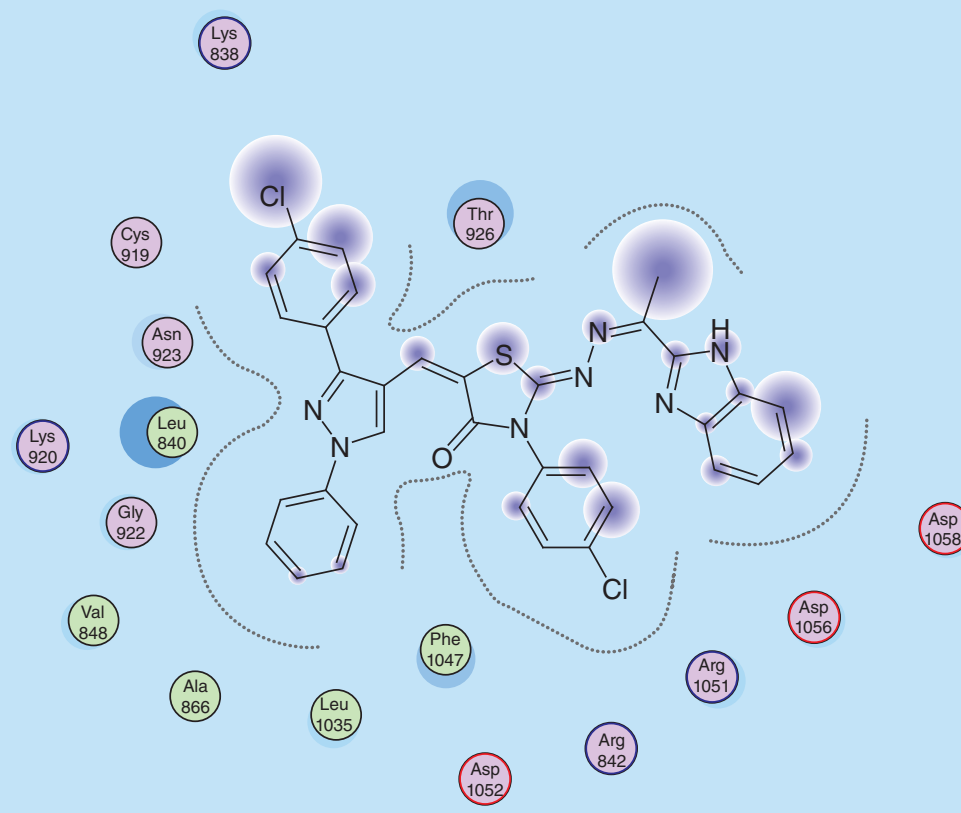

2D ligand-enzyme interaction

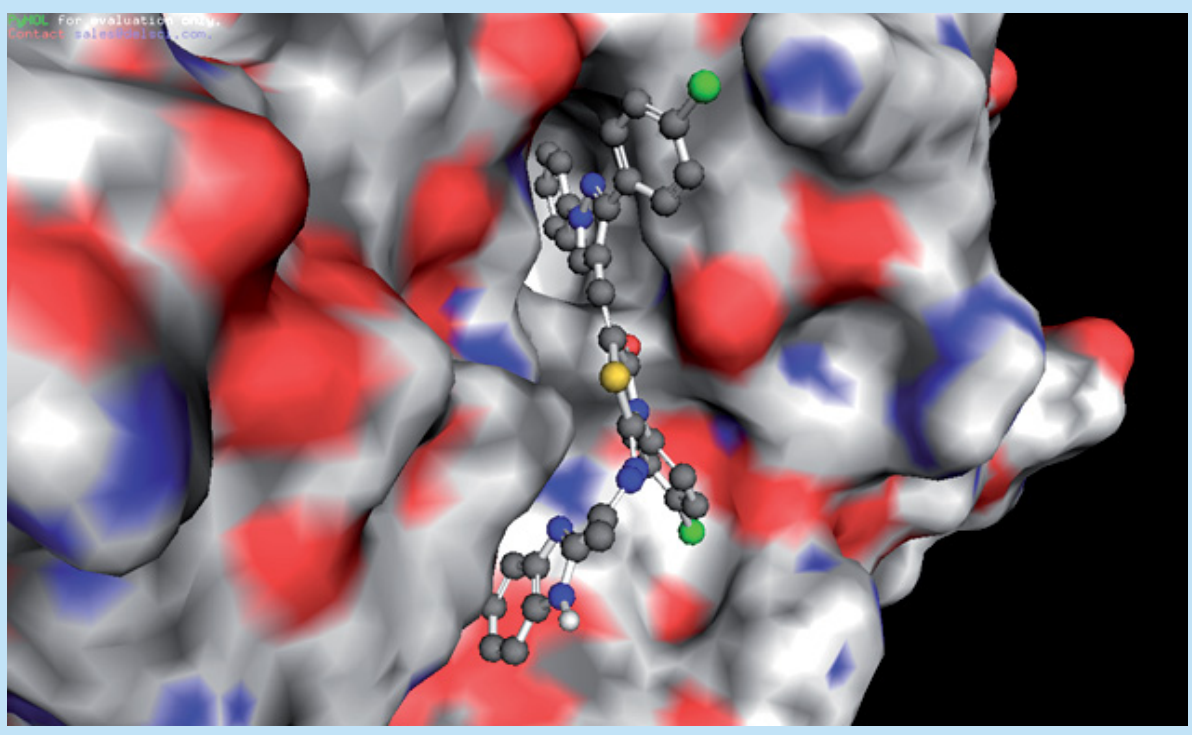

3D ligand-enzyme interaction

Figure 9. Mode of binding of compound $5 q\left(\mathrm{IC}_{50}=35.72 \mu \mathrm{g} / \mathrm{ml}\right.$ and docking score $\left.=-7.74 \mathrm{kcal} / \mathrm{mol}\right)$ inside VEGFR-2 active site.

and thiazolidinone moieties. In addition, the anticancer pharmacophore was planned to contain moieties like 1,3-diaryl-1 $H$-pyrazole or 2-oxoindolin-3-ylidene able to inhibit kinases, specifically tyrosine kinases, to suppress $\mathrm{HCC}$ development, angiogenesis and potentiate the anti-HCV activity by inhibition of NS5A activation. Biological screening results revealed that compounds $\mathbf{5 f}, \mathbf{5 j}, \mathbf{5 1}, \mathbf{5 p}, \mathbf{5 q}, \mathbf{5 r}, \mathbf{6 c}$ and $\mathbf{6 d}$ exhibited potential in vitro anticancer activity against HCC cell line HepG2, while compounds 5a, 51, 5p and 5v showed in vitro anti-HCV activity against HepG2 cells infected with HCV. Consequently, compounds $\mathbf{5 1}$ and $\mathbf{5 p}$ were found to exhibit in vitro dual activity against HCV and HCC. The structures of the dual acting compounds (51 and $\mathbf{5 p}$ ) contained all the 


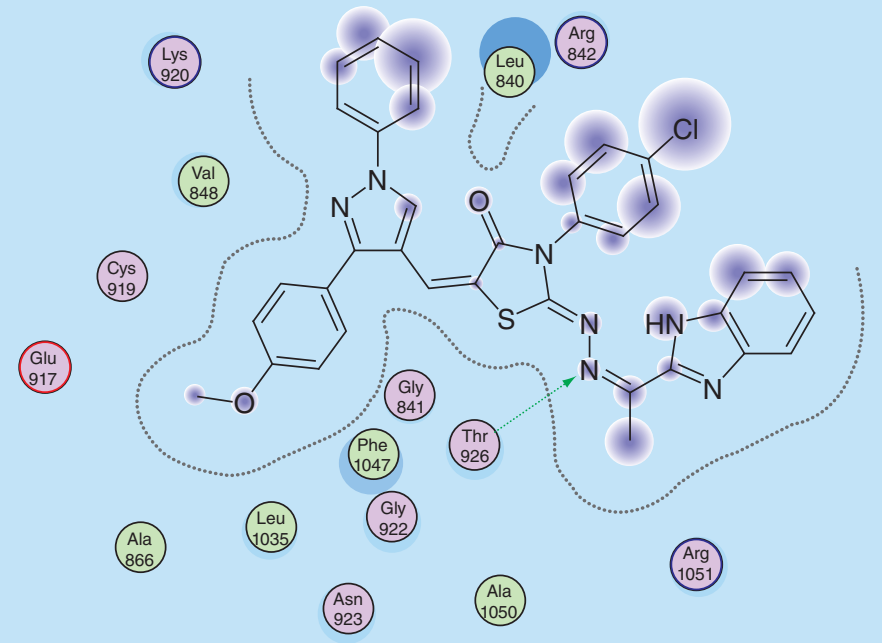

2D ligand-enzyme interaction

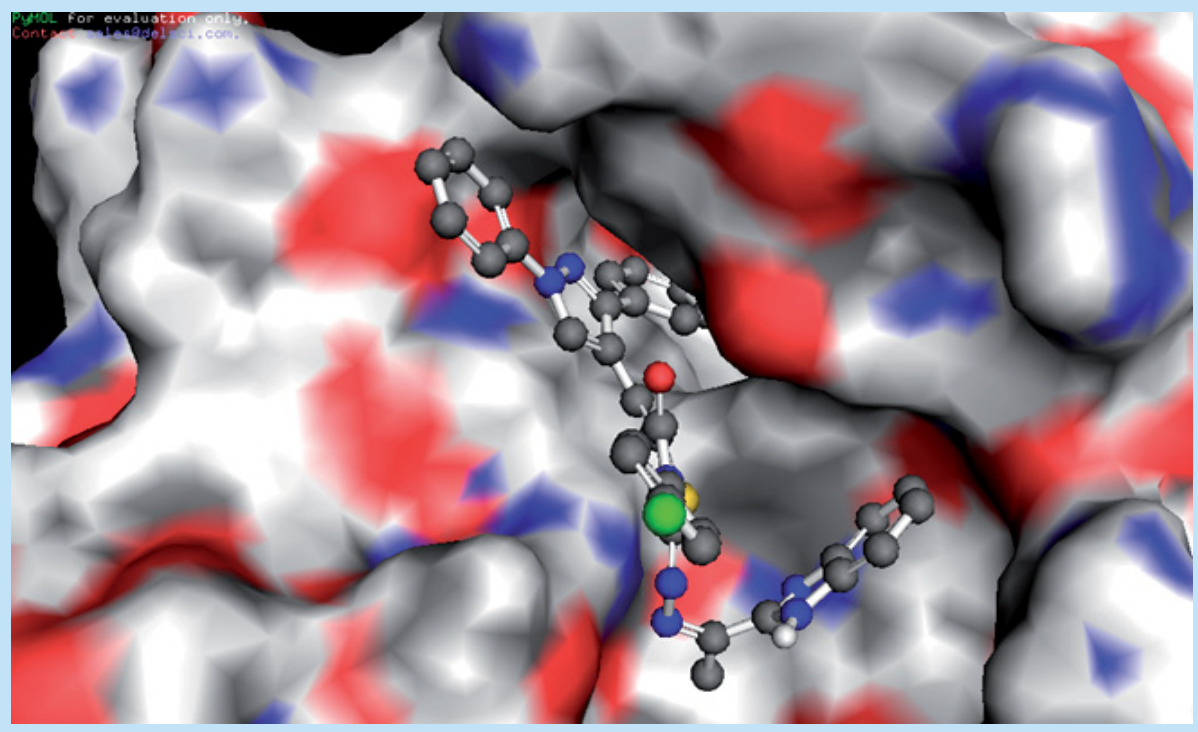

3D ligand-enzyme interaction

Figure 10. Mode of binding of compound $5 \mathrm{r}\left(\mathrm{IC}_{50}=11.01 \mu \mathrm{g} / \mathrm{ml}\right.$, docking score $\left.=-7.37 \mathrm{kcal} / \mathrm{mol}\right)$ inside VEGFR-2 active site.

planned anti-HCV scaffolds like benzofuran 51, benzimidazole $5 p$ and thiazolidinone (51 and $5 p$ ). In addition, all the dual acting compounds contained 1,3-diaryl- $1 H$-pyrazole moiety as anticancer pharmacophore rather than 2-oxoindolin-3-ylidene moiety. These suggested that the combination of the planned anti-HCV scaffolds and 1,3-diaryl-1 $H$-pyrazole moiety could be considered as an optimal platform for further modification to enhance the dual anti-HCV and anticancer activity. During this work, compound $\mathbf{5 q}$ emerged as a promising anticancer lead compound as it showed a remarkable anticancer effect on HepG2 cancer cells with $\mathrm{IC}_{50}$ and $\mathrm{IC}_{100}$ values $<100 \mu \mathrm{g} / \mathrm{ml}$ and less than its $\mathrm{LD}_{0}$ on PBMC normal cells. This compound also showed a very high value of selectivity index indicating high selectivity toward cancerous HepG2 cells. This compound could be considered as a potent, safe and selective anticancer agent against HCC. This compound could be a lead one for further structure modification to achieve more potent anticancer agents. Finally, docking studies suggested that the newly synthesized compounds might suppress HCC through tyrosine kinase inhibition. In addition, inhibiting kinases 


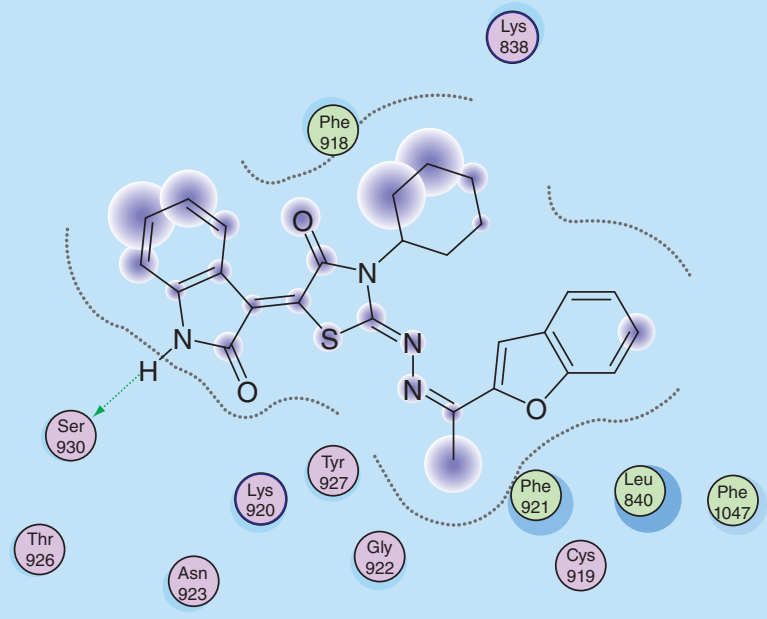

2D ligand-enzyme interaction

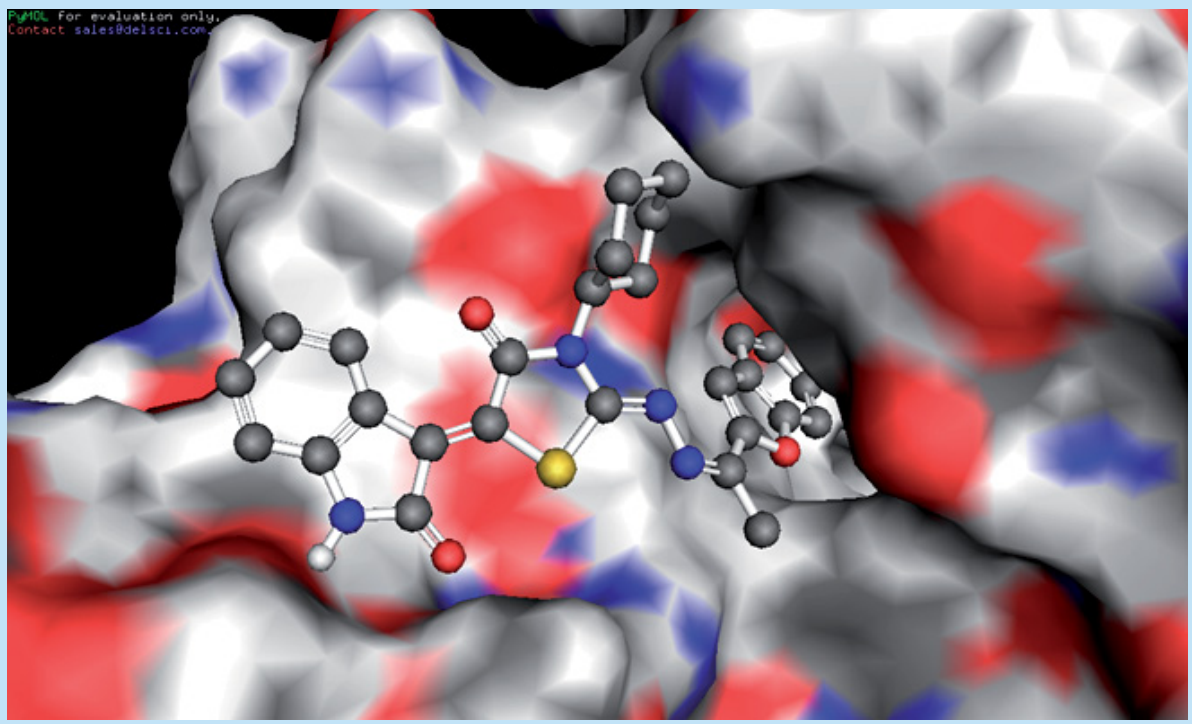

3D ligand-enzyme interaction

Figure 11. Mode of binding of compound $6 \mathrm{~d}\left(\mathrm{IC}_{50}=34.28 \mu \mathrm{g} / \mathrm{ml}\right.$ and docking score $\left.=-7.67 \mathrm{kcal} / \mathrm{mol}\right)$ inside VEGFR-2 active site.

could inhibit phosphorylation (activation) of HCV NS5A enzyme which might add to the in vitro anti-HCV effect of the dual acting compounds.

\section{Experimental}

Chemistry

Melting points were determined in open glass capillaries on a Stuart SMP10 melting point apparatus (Bibby Scientific Ltd, Stone, UK) and were uncorrected. IR spectra were recorded, using $\mathrm{KBr}$ discs $v\left(\mathrm{~cm}^{-1}\right)$, on a PerkinElmer 1430 Infrared spectrophotometer (Central Laboratory, Faculty of Pharmacy, Alexandria University, Egypt). Nuclear magnetic resonance spectra, ${ }^{1} \mathrm{H}-\mathrm{NMR}$ were scanned on a Jeol NMR $500 \mathrm{MHz}$ spectrophotometer (Faculty of Science, Alexandria University and National Research Center, Dokki, Cairo) and Mercury $300 \mathrm{MHz}$ spectrophotometer (Faculty of Science, Cairo University, Egypt). The data were reported as $\delta$-values (p.p.m.) relative to tetramethylsilane as internal standard. The type of signal was indicated by one of the following letters: 
$\mathrm{s}=$ singlet, $\mathrm{d}=$ doublet, $\mathrm{t}=$ triplet, $\mathrm{q}=$ quartet, $\mathrm{m}=$ multiplet, br. = broad, dist. = distorted. Mass spectra [54] were run on a gas chromatograph/mass spectrometer Shimadzu GCMS-Qp2010 plus (70 ev; Faculty of Science, Cairo University, Egypt). The peak intensities, in parentheses, are expressed as percentage abundance. Elemental analyses were performed at the microanalytical unit, Faculty of Pharmacy, Assiut University and at the Regional Center for Mycology and Biotechnology, Al-Azhar University; all the values were within range of \pm 0.4 . Reactions were monitored by thin-layer chromatography on silica gel (60 GF254, Merck, Darmstadt, Germany), using glass plates and the spots were visualized by exposure to iodine vapor or UV-lamp at $1254 \mathrm{~nm}$ for few seconds. All reagents and solvents were obtained from commercial sources, and were purified and dried by standard techniques.

Compounds 1a [55], $\mathbf{1 b}$ [56-61], 2a [39], 2f [62], 2g [62], 2i [62], 3a [39] and 4a-c [63-65] were prepared according to the reported procedures.

\section{General procedure for the synthesis of thiosemicarbazones intermediates $2 a-i$}

To a suspension of 2-acetylbenzofuran or 2-acetylbenzimidazole (1a-b) $(0.8 \mathrm{~g}, 5 \mathrm{mmol})$ and the appropriately substituted thiosemicarbazide $(5 \mathrm{mmol})$ in $10 \mathrm{ml}$ absolute ethanol, few drops of glacial acetic acid was added. The reaction mixture was refluxed for 3-4 h, then allowed to cool to room temperature. The precipitate formed was filtered, washed with ethanol, air dried and recrystallized from ethanol.

\section{1-[1-(Benzofuran-2-yl)ethylideneamino]-3-(4-chlorophenyl)thiourea $2 b$}

Yellowish white solid, yield $84 \%$, melting point (m.p.) $220-2^{\circ} \mathrm{C}$. Fourier-transform infrared spectroscopy (FT-IR) $\left(\mathrm{cm}^{-1}\right): 3230(\mathrm{~N}-\mathrm{H}) ; 1634(\mathrm{C}=\mathrm{N}) ; 1571,1304,1187,927(\mathrm{~N}-\mathrm{C}=\mathrm{S}$ amide I, II, III and IV bands); 1262, 1158, 1093 (C-O-C). ${ }^{1} \mathrm{H}-\mathrm{NMR}$ (DMSO-d ${ }_{6}$, Mercury $\left.300 \mathrm{MHz}\right): \delta 2.43$ (s, 3H, -N = C-CH 3 ); 7.23-7.74 (m, $9 \mathrm{H}, \mathrm{Ar}-\mathrm{C}^{2,3,5,6}-\mathrm{Hs}$ and benzofuran $\left.\mathrm{C}^{3,4,5,6,7}-\mathrm{Hs}\right) ; 10.09,10.93\left(2 \mathrm{~s}\right.$, each $1 \mathrm{H}, 2-\mathrm{NH}$ of thiosemicarbazone, $\mathrm{D}_{2} \mathrm{O}$ exchangeable). Anal. Calcd. for $\mathrm{C}_{17} \mathrm{H}_{14} \mathrm{ClN}_{3} \mathrm{OS}$ : C, 59.38; H, 4.10; N, 12.22. Found: C, 59.51; H, 4.15; N, 12.31 .

\section{1-[1-(Benzofuran-2-yl)ethylideneamino]-3-(4-methoxyphenyl)thiourea $2 c$}

Yellowish white solid, yield 87\%, m.p. 204-6 ${ }^{\circ} \mathrm{C}$. FT-IR $\left(\mathrm{cm}^{-1}\right): 3225(\mathrm{~N}-\mathrm{H}) ; 1627(\mathrm{C}=\mathrm{N}) ; 1569,1302,1182$, 924 (N-C $=$ S amide I, II, III and IV bands); 1259, 1156, 1090 (C-O-C). ${ }^{1} \mathrm{H}-\mathrm{NMR}$ (DMSO-d $\mathrm{d}_{6}$, Mercury $300 \mathrm{MHz}): \delta 2.41\left(\mathrm{~s}, 3 \mathrm{H},-\mathrm{N}=\mathrm{C}-\mathrm{CH}_{3}\right) ; 3.78\left(\mathrm{~s}, 3 \mathrm{H}, \mathrm{O}-\mathrm{CH}_{3}\right) ; 6.94\left(\mathrm{~d}, \mathrm{~J}=9 \mathrm{~Hz}, 2 \mathrm{H}, \mathrm{Ar}-\mathrm{C}^{2,6}-\underline{\mathrm{Hs}}\right) ; 7.24-7.31$ (dist. dd, $1 \mathrm{H}$, benzofuran $\left.\mathrm{C}^{5}-\mathrm{H}\right)$; 7.33-7.39 (dist. dd, 1H, benzofuran $\left.\mathrm{C}^{6}-\underline{\mathrm{H}}\right)$; $7.44\left(\mathrm{~d}, \mathrm{~J}=9 \mathrm{~Hz}, 2 \overline{\mathrm{H}}, \mathrm{Ar}-\mathrm{C}^{3,5}-\mathrm{Hs}\right.$ ); $7.62\left(\mathrm{~d}, \mathrm{~J}=8 \mathrm{~Hz}, 1 \mathrm{H}\right.$, benzofuran $\left.\mathrm{C}^{7}-\underline{\mathrm{H}}\right)$; 7.65-7.70 (m, 2H, benzofuran $\left.\mathrm{C}^{3,4}-\underline{\mathrm{Hs}}\right)$; 9.91, $10.76(2 \mathrm{~s}$, each $\overline{1 \mathrm{H}}$, 2 -NH of thiosemicarbazone, $\mathrm{D}_{2} \mathrm{O}$ exchangeable). Anal. Calcd. for $\mathrm{C}_{18} \mathrm{H}_{17} \mathrm{~N}_{3} \mathrm{O}_{2} \overline{\mathrm{S}}: \mathrm{C}, 63.70 ; \mathrm{H}, 5.05 ; \mathrm{N}, 12.38$. Found: C, 63.83; H, 5.03; N, 12.54 .

\section{1-[1-(Benzofuran-2-yl)ethylideneamino]-3-cyclohexylthiourea 2d}

White solid, yield 90\%, m.p. 182-4 ${ }^{\circ} \mathrm{C}$. FT-IR $\left(\mathrm{cm}^{-1}\right)$ : $3218(\mathrm{~N}-\mathrm{H}) ; 1629(\mathrm{C}=\mathrm{N}) ; 1568,1305,1187,922(\mathrm{~N}-$ $\mathrm{C}=\mathrm{S}$ amide I, II, III and IV bands); 1258, 1151, 1091 (C-O-C). ${ }^{1} \mathrm{H}-\mathrm{NMR}$ (DMSO-d $\mathrm{d}_{6}$, Mercury $\left.300 \mathrm{MHz}\right): \delta$ $1.12-1.51,1.54-1.78$ and $1.86-1.99\left(3 \mathrm{~m}, 10 \mathrm{H}\right.$, cyclohexyl $\left.\mathrm{C}^{2,3,4,5,6}-\mathrm{H}_{2}\right) ; 2.34\left(\mathrm{~s}, 3 \mathrm{H},-\mathrm{N}=\mathrm{C}-\mathrm{CH}_{3}\right) ; 4.11-4.30$ $\left(\mathrm{m}, 1 \mathrm{H}\right.$, cyclohexyl $\left.\mathrm{C}^{1}-\underline{\mathrm{H}}\right) ; 7.24-7.31$ (dist. dd, $1 \mathrm{H}$, benzofuran $\left.\mathrm{C}^{5}-\underline{\mathrm{H}}\right) ; 7.32-7.40$ (dist. dd, $1 \mathrm{H}$, benzofuran $\mathrm{C}^{6}$ $\underline{\mathrm{H}}$ ); $7.52\left(\mathrm{~s}, 1 \mathrm{H}\right.$, benzofuran $\left.\mathrm{C}^{3}-\underline{\mathrm{H}}\right)$; 7.58-7.63 (dist. d, $1 \mathrm{H}$, benzofuran $\mathrm{C}^{7}-\underline{\mathrm{H}}$ ); 7.66-7.71 (dist. d, $1 \mathrm{H}$, benzofuran $\left.\overline{\mathrm{C}}^{4}-\mathrm{H}\right) ; 7.99\left(\mathrm{~d}, \mathrm{~J}=8.5 \mathrm{~Hz}, 1 \overline{\mathrm{H}},-\mathrm{NH}-\right.$ cyclohexyl, $\mathrm{D}_{2} \mathrm{O}$ exchangeable); 10.43 (s, $1 \mathrm{H},-\mathrm{NH}$ of thiosemicarbazide, $\mathrm{D}_{2} \overline{\mathrm{O}}$ exchangeable). Anal. Calcd. for $\overline{\mathrm{C}}_{17} \mathrm{H}_{21} \mathrm{~N}_{3} \mathrm{OS} .1 / 2 \mathrm{H}_{2} \mathrm{O}: \mathrm{N}, 12.95 ;$ S, 9.88. Found: $\overline{\mathrm{N}}, 13.20$; S, 9.47 .

\section{1-[1-(1H-Benzimidazole-2-yl)ethylideneamino]-3-phenylthiourea 2e}

White solid, yield 92\%, m.p. 176-8 ${ }^{\circ}$ C. FT-IR $\left(\mathrm{cm}^{-1}\right): 3340(\mathrm{~N}-\mathrm{H}) ; 1625(\mathrm{C}=\mathrm{N}) ; 1569,1301,1184,923$ $\left(\mathrm{N}-\mathrm{C}=\mathrm{S}\right.$ amide I, II, III and IV bands). ${ }^{1} \mathrm{H}-\mathrm{NMR}\left(\mathrm{DMSO}_{\mathrm{d}}\right.$, Mercury $\left.300 \mathrm{MHz}\right): \delta 2.53(\mathrm{~s}, 3 \mathrm{H},-\mathrm{N}=\mathrm{C}-$ $\left.\mathrm{CH}_{3}\right) ; 7.16-7.59$ (m, 8H, benzimidazole $\mathrm{C}^{4,5,6}-\underline{\mathrm{Hs}}$ and $\left.\mathrm{Ar}-\mathrm{C}^{2,3,4,5,6}-\underline{\mathrm{Hs}}\right) ; 7.70$ (d, J $=7.8 \mathrm{~Hz}$, benzimidazole $\left.\mathrm{C}^{7}-\underline{\mathrm{H}}\right) ; 10.43,11.02\left(2 \mathrm{~s}\right.$, each $1 \mathrm{H}, 2-\mathrm{NH}$ of thiosemicarbazone, $\overline{\mathrm{D}_{2} \mathrm{O}}$ exchangeable); $12.84(\mathrm{~s}, 1 \mathrm{H},-\mathrm{NH}$ of benzimidazole, $\mathrm{D}_{2} \mathrm{O}$ exchangeable). Anal. Calcd. for $\mathrm{C}_{16} \mathrm{H}_{15} \mathrm{~N}_{5} \mathrm{~S}$ : C, 62.11; H, 4.89; N, 22.64. Found: C, 62.17; H, 4.93; N, 22.81 .

\section{1-[1-(1H-Benzimidazole-2-yl)ethylideneamino]-3-(4-methylphenyl)thiourea $2 \mathrm{~h}$}

Yellowish white solid, yield 83\%, m.p. $184-6^{\circ} \mathrm{C}$. FT-IR $\left(\mathrm{cm}^{-1}\right): 3325(\mathrm{~N}-\mathrm{H}) ; 1618(\mathrm{C}=\mathrm{N}) ; 1563,1298,1190$, 926 (N-C $=S$ amide I, II, III and IV bands). Anal. Calcd. for $\mathrm{C}_{17} \mathrm{H}_{17} \mathrm{~N}_{5} \mathrm{~S}: \mathrm{C}, 63.13 ; \mathrm{H}, 5.30 ; \mathrm{N}, 21.65$. Found: C, 63.22; H, 5.28; N, 21.78 . 
General procedure for the synthesis of 4-thiazolidinone intermediates 3a-i

A mixture of 1-[1-(benzofuran-2-yl)ethylideneamino]-3-substituted thioureas or 1-[1-(1H-benzimidazol-2yl)ethylideneamino]-3-substituted thioureas (2a-i) $(5 \mathrm{mmol})$, monochloroacetic acid (0.65 g, $7.5 \mathrm{mmol})$ and anhydrous sodium acetate $(0.62 \mathrm{~g}, 7.5 \mathrm{mmol})$ in glacial acetic acid $(10 \mathrm{ml})$ was heated under reflux for $5-6 \mathrm{~h}$, left to attain room temperature, then poured onto crushed ice. The precipitate formed was filtered, washed with water and crystallized from ethanol.

2-[1-(Benzofuran-2-yl)ethylidenehydrazono]-3-(4-chlorophenyl)thiazolidin-4-one 3b

Yellow solid, yield 72\%, m.p. 254-6 ${ }^{\circ}$ C. FT-IR $\left(\mathrm{cm}^{-1}\right): 1726(\mathrm{C}=\mathrm{O}) ; 1606(\mathrm{C}=\mathrm{N}) ; 1259,1171,1087(\mathrm{C}-\mathrm{O}-\mathrm{C})$. ${ }^{1} \mathrm{H}-\mathrm{NMR}\left(\mathrm{DMSO}-\mathrm{d}_{6}\right.$, Mercury $\left.300 \mathrm{MHz}\right): \delta 2.18\left(\mathrm{~s}, 3 \mathrm{H},-\mathrm{N}=\mathrm{C}-\mathrm{CH}_{3}\right) ; 4.11\left(\mathrm{~s}, 2 \mathrm{H}\right.$, thiazolidinone $\left.\mathrm{C}^{5}-\mathrm{H}_{2}\right)$; 7.25-7.32 (dist. dd, $1 \mathrm{H}$, benzofuran $\mathrm{C}^{5}-\mathrm{H}$ ); 7.36-7.42 (dist. dd, $1 \mathrm{H}$, benzofuran $\left.\mathrm{C}^{6}-\mathrm{H}\right) ; 7.44$ (s, 1H, benzofuran $\left.\mathrm{C}^{3}-\mathrm{H}\right) ; 7.48\left(\mathrm{~d}, \mathrm{~J}=8.6 \mathrm{~Hz}, \mathrm{Ar}-\mathrm{C}^{2,6}-\mathrm{Hs}\right) ; 7.61\left(\mathrm{~d}, \mathrm{~J}=8.6 \mathrm{~Hz}, \mathrm{Ar}-\mathrm{C}^{3,5}-\mathrm{Hs}\right) ; 7.65$ (d, $\overline{\mathrm{J}}=7.7 \mathrm{~Hz}, 1 \mathrm{H}$, benzofuran $\left.\mathrm{C}^{7}-\overline{\mathrm{H}}\right) ; 7.71$ (d, J = 7.7 Hz, $1 \mathrm{H}$, benzofuran $\left.\mathrm{C}^{4}-\mathrm{H}\right)$. Anal. Calcd. for $\overline{\mathrm{C}}_{19} \mathrm{H}_{14} \mathrm{ClN}_{3} \mathrm{O}_{2} \mathrm{~S}: \mathrm{C}, 59.45 ; \mathrm{H}, 3.68 ; \mathrm{N}$, 10.95. Found: C, 59.59; H, 3.74; N, 11.12 .

2-[1-(Benzofuran-2-yl)ethylidenehydrazono]-3-(4-methoxyphenyl)thiazolidin-4-one 3c

Yellow solid, yield 73\%, m.p. 239-41 ${ }^{\circ}$ C. FT-IR $\left(\mathrm{cm}^{-1}\right)$ : $1722(\mathrm{C}=\mathrm{O}) ; 1610(\mathrm{C}=\mathrm{N}) ; 1257,1170,1082(\mathrm{C}-$ O-C). ${ }^{1} \mathrm{H}-\mathrm{NMR}$ (DMSO-d 6 , Mercury $\left.300 \mathrm{MHz}\right): \delta 2.18\left(\mathrm{~s}, 3 \mathrm{H},-\mathrm{N}=\mathrm{C}-\mathrm{CH}_{3}\right) ; 3.82\left(\mathrm{~s}, 3 \mathrm{H}, \mathrm{O}-\mathrm{CH}_{3}\right) ; 4.09(\mathrm{~s}$, $2 \mathrm{H}$, thiazolidinone $\left.\mathrm{C}^{5}-\mathrm{H}_{2}\right) ; 7.06\left(\mathrm{~d}, \mathrm{~J}=9 \mathrm{~Hz}, 2 \mathrm{H}, \mathrm{Ar}-\mathrm{C}^{3,5}-\mathrm{Hs}\right) ; 7.25-7.4 \overline{1}\left(\mathrm{~m}, 4 \mathrm{H}\right.$, benzofuran $\overline{\mathrm{C}}^{5,6}-\mathrm{Hs}$ and

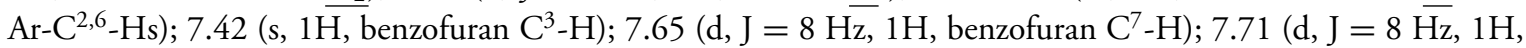
benzofuran $\left.\mathrm{C}^{4}-\underline{\mathrm{H}}\right)$. Anal. Calcd. for $\mathrm{C}_{20} \overline{\mathrm{H}}_{17} \mathrm{~N}_{3} \mathrm{O}_{3} \mathrm{~S}$ : C, 63.31; H, 4.52; N, 11.07. Found: C, 63.44; H, 4.59; N, 11.32 .

2-[1-(Benzofuran-2-yl)ethylidenehydrazono]-3-cyclohexylthiazolidin-4-one 3d

Yellow solid, yield 97\%, m.p. 172-4 ${ }^{\circ}$ C. FT-IR $\left(\mathrm{cm}^{-1}\right): 1724(\mathrm{C}=\mathrm{O}) ; 1609(\mathrm{C}=\mathrm{N}) ; 1256,1173,1083(\mathrm{C}-\mathrm{O}-$ C). ${ }^{1} \mathrm{H}-\mathrm{NMR}\left(\mathrm{DMSO}_{6}\right.$, Mercury $\left.300 \mathrm{MHz}\right): \delta 1.01-1.45,1.57-1.72,1.77-1.90$ and $2.28-2.42(4 \mathrm{~m}, 10 \mathrm{H}$, cyclohexyl $\left.\mathrm{C}^{2,3,4,5,6}-\mathrm{H}_{2}\right) ; 2.43\left(\mathrm{~s}, 3 \mathrm{H},-\mathrm{N}=\mathrm{C}-\mathrm{CH}_{3}\right) ; 3.94\left(\mathrm{~s}, 2 \mathrm{H}\right.$, thiazolidinone $\left.\mathrm{C}^{5}-\mathrm{H}_{2}\right) ; 4.27-4.40(\mathrm{~m}, 1 \mathrm{H}$, cyclohexyl $\mathrm{C}^{1}-\underline{\mathrm{H}}$ ); $7.25-7.32$ (dist. dd, $1 \mathrm{H}$, benzofuran $\mathrm{C}^{5}-\underline{\mathrm{H}}$ ); 7.35-7.43 (dist. dd, $1 \mathrm{H}$, benzofuran $\mathrm{C}^{6}-\underline{\mathrm{H}}$ ); 7.47 $\left(\mathrm{s}, 1 \mathrm{H}\right.$, benzofuran $\left.\mathrm{C}^{3}-\mathrm{H}\right) ; 7.66\left(\mathrm{~d}, \mathrm{~J}=8 \mathrm{~Hz}, 1 \mathrm{H}\right.$, benzofuran $\left.\mathrm{C}^{7}-\mathrm{H}\right) ; 7.72\left(\mathrm{~d}, \mathrm{~J}=8 \mathrm{~Hz}, 1 \mathrm{H}\right.$, benzofuran $\left.\mathrm{C}^{4}-\mathrm{H}\right)$. Anal. Calcd. for $\mathrm{C}_{19} \mathrm{H}_{21} \mathrm{~N}_{3} \mathrm{O}_{2} \mathrm{~S}$ : C, 64.20; H, 5.95; N, 11.82; S, 9.02 . Found: C, 64.19; H, 5.83; N, $11.75 ; \mathrm{S}$, 8.71 .

2-[1-(1H-Benzimidazole-2-yl)ethylidenehydrazono]-3-phenylthiazolidin-4-one 3e

Yellow solid, yield 81\%, m.p. $258-60^{\circ} \mathrm{C}$. FT-IR $\left(\mathrm{cm}^{-1}\right): 3066(\mathrm{~N}-\mathrm{H}) ; 1728(\mathrm{C}=\mathrm{O}) ; 1598(\mathrm{C}=\mathrm{N}) .{ }^{1} \mathrm{H}-\mathrm{NMR}$ (DMSO-d ${ }_{6}$, Mercury $\left.300 \mathrm{MHz}\right): \delta 2.26\left(\mathrm{~s}, 3 \mathrm{H},-\mathrm{N}=\mathrm{C}-\mathrm{CH}_{3}\right) ; 4.17$ (s, 2H, thiazolidinone $\left.\mathrm{C}^{5}-\mathrm{H}_{2}\right) ; 7.18-7.29$ (m, 2H, benzimidazole $\left.\mathrm{C}^{5,6}-\underline{\mathrm{Hs}}\right) ; 7.38-7.55\left(\mathrm{~m}, 5 \mathrm{H}, \mathrm{Ar}-\mathrm{C}^{2,3, \overline{4,5}, 6}-\underline{\mathrm{Hs}}\right) ; 7.59-7.64\left(\mathrm{~m}, 2 \mathrm{H}\right.$, benzimidazole $\left.\mathrm{C}^{4,7}-\underline{\mathrm{Hs}}\right)$; 12.50 (s, $1 \mathrm{H},-\mathrm{NH}$ of benzimidazole, $\mathrm{D}_{2} \mathrm{O}$ exchangeable). Anal. $\overline{\mathrm{C}}$ alcd. for $\mathrm{C}_{18} \mathrm{H}_{15} \mathrm{~N}_{5} \mathrm{OS}$ : C, 61.87; $\mathrm{H}, 4.33 ; \mathrm{N}$, 20.04. Found: C, 61.49; H, 4.39; N, 20.20.

2-[1-(1H-Benzimidazole-2-yl)ethylidenehydrazono]-3-(4-chlorophenyl)thiazolidin-4-one $3 f$

Yellow solid, yield 65\%, m.p. $249-51^{\circ} \mathrm{C}$. FT-IR $\left(\mathrm{cm}^{-1}\right): 3050(\mathrm{~N}-\mathrm{H}) ; 1725(\mathrm{C}=\mathrm{O}) ; 1601(\mathrm{C}=\mathrm{N}) .{ }^{1} \mathrm{H}$ NMR (DMSO-d $\mathrm{d}_{6}$, Mercury $\left.300 \mathrm{MHz}\right): \delta 2.28\left(\mathrm{~s}, 3 \mathrm{H},-\mathrm{N}=\mathrm{C}-\mathrm{CH}_{3}\right) ; 4.16\left(\mathrm{~s}, 2 \mathrm{H}\right.$, thiazolidinone $\left.\mathrm{C}^{5}-\mathrm{H}_{2}\right)$; 7.21-7.28 (m, 2H, benzimidazole $\left.\mathrm{C}^{5,6}-\mathrm{Hs}\right) ; 7.50\left(\mathrm{~d}, \mathrm{~J}=8.7 \mathrm{~Hz}, \overline{2 \mathrm{H}}, \mathrm{Ar}-\mathrm{C}^{2,6}-\mathrm{Hs}\right) ; 7.58-7.65(\mathrm{~m}, 4 \mathrm{H}, \overline{\mathrm{Ar}}-$ $\mathrm{C}^{3,5}$-Hs and benzimidazole $\left.\mathrm{C}^{4,7}-\mathrm{Hs}\right) ; 12 . \overline{50}\left(\mathrm{~s}, 1 \mathrm{H},-\mathrm{NH}\right.$ of benzimidazole, $\mathrm{D}_{2} \mathrm{O}$ exchangeable). Anal. Calcd. for $\mathrm{C}_{18} \overline{\mathrm{H}}_{14} \mathrm{ClN}_{5} \mathrm{OS} .1 / 2 \mathrm{H}_{2} \mathrm{O}$ : C, 55.03; H, 3.85; N, 17.83; S, 8.16. Found: C, 55.12; H, 4.03; N, $17.74 ;$ S, 8.10.

2-[1-(1H-Benzimidazole-2-yl)ethylidenehydrazono]-3-(4-methoxyphenyl)thiazolidin-4-one 3g

Yellow solid, yield 84\%, m.p. $253-5^{\circ}$ C. FT-IR $\left(\mathrm{cm}^{-1}\right)$ : $3044(\mathrm{~N}-\mathrm{H}) ; 1729(\mathrm{C}=\mathrm{O}) ; 1603(\mathrm{C}=\mathrm{N}) .{ }^{1} \mathrm{H}-\mathrm{NMR}$ $\left(\mathrm{DMSO}_{6}, \mathrm{~d}_{6}\right.$, Mercury $\left.300 \mathrm{MHz}\right): \delta 2.27\left(\mathrm{~s}, 3 \mathrm{H},-\mathrm{N}=\mathrm{C}-\mathrm{CH}_{3}\right) ; 3.81$ (s, 3H, O-CH ); 4.14 (s, 2H, thiazolidinone $\left.\mathrm{C}^{5}-\mathrm{H}_{2}\right) ; 7.06$ (d, J = 8.5 Hz, 2H, Ar-C $\mathrm{C}^{3,5}-\mathrm{Hs}$ ); 7.16-7.23 (dist. dd, 1H, benzimidazole $\mathrm{C}^{5}-\mathrm{H}$ ); $7.24-7.31$ (dist. $\mathrm{dd}, \overline{1 \mathrm{H}}$, benzimidazole $\left.\mathrm{C}^{6}-\mathrm{H}\right) ; 7.35\left(\mathrm{~d}, \mathrm{~J}=8.5 \mathrm{~Hz}, 2 \mathrm{H}, \mathrm{Ar}-\mathrm{C}^{2,6}-\mathrm{Hs}\right) ; 7.56(\mathrm{~d}, \mathrm{~J}=8 \mathrm{~Hz}, 1 \mathrm{H}$, benzimidazole $\left.\mathrm{C}^{4}-\mathrm{H}\right) ; 7.68\left(\mathrm{~d}, \mathrm{~J}=8 \mathrm{~Hz}, \overline{\mathrm{H}}\right.$, benzimidazole $\left.\mathrm{C}^{7}-\mathrm{H}\right) ; 12.50$ (s, 1H, - $\mathrm{NH}$ of benzimidazole, $\mathrm{D}_{2} \mathrm{O}$ exchangeable). Anal. Calcd. for $\mathrm{C}_{19} \mathrm{H}_{17} \mathrm{~N}_{5} \mathrm{O}_{2} \mathrm{~S}$ : C, 60.14; H, 4.52; N, 18.46; S, 8.45. Found: C, 60.05; H, 4.46; N, 18.31; S, 8.44 . 
2-[1-(1H-Benzimidazole-2-yl)ethylidenehydrazono]-3-(4-methylphenyl)thiazolidin-4-one 3h

Yellow solid, yield 85\%, m.p. 251-3 ${ }^{\circ} \mathrm{C}$. FT-IR $\left(\mathrm{cm}^{-1}\right)$ : $3052(\mathrm{~N}-\mathrm{H}) ; 1727(\mathrm{C}=\mathrm{O}) ; 1609(\mathrm{C}=\mathrm{N}) .{ }^{1} \mathrm{H}-\mathrm{NMR}$ $\left(\mathrm{CDCl}_{3}\right.$, Jeol $\left.500 \mathrm{MHz}\right): \delta 1.59$ (s, 3H, p- $\mathrm{CH}_{3}$ phenyl); 2.43 (s, 3H, $\left.-\mathrm{N}=\mathrm{C}^{-} \mathrm{CH}_{3}\right) ; 4.01$ (s, 2H, thiazolidinone

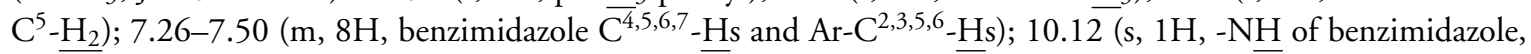
$\mathrm{D}_{2} \mathrm{O}$ exchangeable). Electron-impact mass spectrum (EIMS) $\mathrm{m} / \mathrm{z}$ (\% abundance), 365 (4.10) $\mathrm{M}^{+}+2 ; 364$ (13.56) $\mathrm{M}^{+}+1 ; 363(51.62) \mathrm{M}^{+} ; 118$ (100). Anal. Calcd. for $\mathrm{C}_{19} \mathrm{H}_{17} \mathrm{~N}_{5} \mathrm{OS}$ : C, 62.79; $\mathrm{H}, 4.71 ; \mathrm{N}, 19.27$. Found: C, 62.88; H, 4.69; N, 19.41 .

2-[1-(1H-Benzimidazole-2-yl)ethylidenehydrazono]-3-cyclohexylthiazolidin-4-one $3 i$

Yellow solid, yield 83\%, m.p. 229-31 ${ }^{\circ} \mathrm{C}$. FT-IR $\left(\mathrm{cm}^{-1}\right)$ : $3035(\mathrm{~N}-\mathrm{H}) ; 1724(\mathrm{C}=\mathrm{O}) ; 1599(\mathrm{C}=\mathrm{N}) .{ }^{1} \mathrm{H}$ NMR (DMSO-d $\mathrm{d}_{6}$, Mercury $\left.300 \mathrm{MHz}\right): \delta 1.12-1.42,1.59-1.72,1.78-1.90$ and $2.28-2.45$ (4m, 10H, cyclohexyl $\left.\mathrm{C}^{2,3,4,5,6}-\underline{\mathrm{H}_{2}}\right) ; 2.53\left(\mathrm{~s}, 3 \mathrm{H},-\mathrm{N}=\mathrm{C}-\mathrm{CH}_{3}\right) ; 4.00\left(\mathrm{~s}, 2 \mathrm{H}\right.$, thiazolidinone $\left.\mathrm{C}^{5}-\underline{\mathrm{H}_{2}}\right) ; 4.27-4.42(\mathrm{~m}, 1 \mathrm{H}$, cyclohexyl $\left.\mathrm{C}^{1}-\underline{\mathrm{H}}\right) ; 7.16-7.24$ (dist. dd, $1 \mathrm{H}$, benzimidazole $\left.\mathrm{C}^{5}-\underline{\mathrm{H}}\right) ; 7.24-7.31$ (dist. dd, $\overline{1 \mathrm{H}}$, benzimidazole $\left.\mathrm{C}^{6}-\underline{\mathrm{H}}\right) ; 7.56(\mathrm{~d}$, $\mathrm{J}=\overline{8} \mathrm{~Hz}, 1 \mathrm{H}$, benzimidazole $\left.\mathrm{C}^{7}-\mathrm{H}\right) ; 7.69\left(\mathrm{~d}, \mathrm{~J}=8 \mathrm{~Hz}, 1 \mathrm{H}\right.$, benzimidazole $\left.\mathrm{C}^{4}-\mathrm{H}\right) ; 12.43(\mathrm{~s}, \overline{\mathrm{H}},-\mathrm{NH}$ of benzimidazole, $\mathrm{D}_{2} \mathrm{O}$ exchangeable). Anal. Calcd. for $\mathrm{C}_{18} \mathrm{H}_{21} \mathrm{~N}_{5} \mathrm{OS}$ : C, 60.82; H, 5.95; N, 19.70; S, 9.02. Found: C, 60.95; H, 5.75; N, 19.54; S, 8.81.

General procedure for the synthesis of compounds $5 a-x$

To a mixture of 2-[1-(benzofuran-2-yl)ethylidenehydrazono]-3-substituted thiazolidin-4-ones or 2-[1-(1Hbenzimidazol-2-yl)ethylidenehydrazono]-3-substituted thiazolidin-4-ones (3a-i) $(1 \mathrm{mmol})$ and 3-(4-substituted phenyl)-1-phenyl-1 $H$-pyrazole-4-carbaldehydes (4a-c) $(1.1 \mathrm{mmol})$ in dry dioxane $(5 \mathrm{ml})$, two drops of piperidine were added. The reaction mixture was refluxed for $8-15 \mathrm{~h}$, concentrated, then allowed to cool to room temperature. The precipitate formed after addition of ethanol $(10 \mathrm{ml})$ was filtered, washed with ethanol and recrystallized from dioxane/ethanol.

\section{2-[1-(Benzofuran-2-yl)ethylidenehydrazono]-5-[(1,3-Diphenyl-1H-pyrazol-4-yl)methylene]-3-phenylthiazolidin-4-one $5 a$}

Yellow solid, yield 80\%, m.p. 204-6 ${ }^{\circ}$ C. FT-IR $\left(\mathrm{cm}^{-1}\right)$ : $1700(\mathrm{C}=\mathrm{O}) ; 1599(\mathrm{C}=\mathrm{N}) ; 1221,1166,1070$ (C-O-C). ${ }^{1} \mathrm{H}-\mathrm{NMR}$ (DMSO-d ${ }_{6}$, Mercury $\left.300 \mathrm{MHz}\right): \delta 2.25\left(\mathrm{~s}, 3 \mathrm{H},-\mathrm{N}=\mathrm{C}_{-} \mathrm{CH}_{3}\right) ; 7.24-7.75(\mathrm{~m}, 19 \mathrm{H}$, benzofuran $\mathrm{C}^{3,4,5,6,7}-\mathrm{Hs}, \mathrm{C}^{2,3,4,5,6}-\mathrm{Hs}$ of thiazolidinone $\mathrm{N}^{3}$-phenyl, $\mathrm{C}^{3,4,5}$ - Hs of pyrazole $\mathrm{C}^{3}$-phenyl, $\mathrm{C}^{2,3,4,5,6}-\mathrm{Hs}$ of pyrazole $\mathrm{N}^{1}$-phenyl and $-\mathrm{C}=\overline{\mathrm{CH}}$ vinylic); $8.05\left(\mathrm{~d}, \mathrm{~J}=8.2 \mathrm{~Hz}, 2 \mathrm{H}, \mathrm{C}^{2,6}-\mathrm{Hs}\right.$ of pyrazole $\mathrm{C}^{3}$-phenyl); $8.95(\overline{\mathrm{s}, 1} \mathrm{H}$, pyrazole $\mathrm{C}^{5}-\underline{\mathrm{H}}$. Anal. Calcd. for $\mathrm{C}_{35} \mathrm{H}_{25} \mathrm{~N}_{5} \mathrm{O}_{2} \mathrm{~S}$ : C, 72.52; H, 4.35; N, 12.08. Found: C, 72.64; H, 4.41; N, 12.21 .

\section{2-[1-(Benzofuran-2-yl)ethylidenehydrazono]-5-\{[3-(4-chlorophenyl)-1-phenyl-1H-pyrazol-4-yl]methylene $\}-3-$ phenylthiazolidin-4-one \\ 56}

Yellow solid, yield 77\%, m.p. 237-9 ${ }^{\circ}$ C. FT-IR $\left(\mathrm{cm}^{-1}\right)$ : $1703(\mathrm{C}=\mathrm{O}) ; 1598(\mathrm{C}=\mathrm{N}) ; 1222,1166,1075$ (C-OC). ${ }^{1} \mathrm{H}-\mathrm{NMR}$ (DMSO-d $\mathrm{d}_{6}$, Mercury $\left.300 \mathrm{MHz}\right): \delta 2.24\left(\mathrm{~s}, 3 \mathrm{H},-\mathrm{N}=\mathrm{C}-\mathrm{CH}_{3}\right) ; 7.26-7.75(\mathrm{~m}, 18 \mathrm{H}$, benzofuran $\mathrm{C}^{3,4,5,6,7}-\mathrm{Hs}, \mathrm{C}^{2,3,4,5,6}-\underline{\mathrm{H} s}$ of thiazolidinone $\mathrm{N}^{3}$-phenyl, $\mathrm{C}^{2,3,4,5,6}$ - Hs of pyrazole $\mathrm{N}^{1}$-phenyl, $\mathrm{C}^{3,5}$-Hs of p-Cl-phenyl

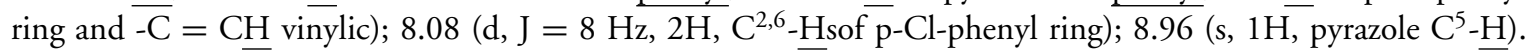
Anal. Calcd. for $\overline{\mathrm{C}_{35}} \mathrm{H}_{24} \mathrm{ClN}_{5} \mathrm{O}_{2} \mathrm{~S}$ : C, 68.45; H, 3.94; N, $\overline{11} .40$. Found: C, 68.53; H, 3.90; N, 11.56.

2-[1-(Benzofuran-2-yl)ethylidenehydrazono]-5-\{[3-(4-methoxyphenyl)-1-phenyl-1H-pyrazol-4-yl]methylene $\}-3-$ phenylthiazolidin-4-one

$5 c$

Yellow solid, yield 90\%, m.p. 187-9 C. FT-IR $\left(\mathrm{cm}^{-1}\right)$ : $1704(\mathrm{C}=\mathrm{O}) ; 1601(\mathrm{C}=\mathrm{N}) ; 1225,1170,1079$ (CO-C). ${ }^{1} \mathrm{H}-\mathrm{NMR}$ (DMSO-d 6 , Mercury $300 \mathrm{MHz}$ ): $\delta 2.24\left(\mathrm{~s}, 3 \mathrm{H},-\mathrm{N}=\mathrm{C}-\mathrm{CH}_{3}\right.$ ); $3.83\left(\mathrm{~s}, 3 \mathrm{H}, \mathrm{O}_{-} \mathrm{CH}_{3}\right) ; 7.12$ $\left(\mathrm{d}, \mathrm{J}=8.5 \mathrm{~Hz}, 2 \mathrm{H}, \mathrm{C}^{3,5}-\mathrm{Hs}\right.$ of $\mathrm{p}-\mathrm{OCH}_{3}$-phenyl ring); 7.26-7.36 (dist. dd, $1 \overline{\mathrm{H}}$, benzofuran $\mathrm{C}^{5}-\mathrm{H}$ ); $7.37-7.71$ $\left(\mathrm{m}, 14 \mathrm{H}\right.$, benzofuran $\mathrm{C}^{3,6,7}-\mathrm{Hs}, \mathrm{C}^{2,3,4,5,6}-\mathrm{Hs}$ of thiazolidinone $\mathrm{N}^{3}$-phenyl, $\mathrm{C}^{2,3,4,5,6}-\mathrm{Hs}$ of pyrazole $\overline{\mathrm{N}}^{1}$-phenyl and $-\mathrm{C}=\mathrm{CH}$ vinylic); $7.73\left(\mathrm{~d}, \overline{\mathrm{J}}=7.5 \mathrm{~Hz}, \overline{1 \mathrm{H}}\right.$, benzofuran $\left.\mathrm{C}^{4}-\underline{\mathrm{H}}\right) ; 8.06\left(\mathrm{~d}, \mathrm{~J}=8.5 \overline{\mathrm{Hz}}, 2 \mathrm{H}, \mathrm{C}^{2,6}-\underline{\mathrm{Hs}}\right.$ of $\mathrm{p}-\mathrm{OCH}_{3}-$ phenyl ring); 8.90 (s, $1 \mathrm{H}$, pyrazole $\left.\mathrm{C}^{5}-\underline{\mathrm{H}}\right)$. Anal. Calcd. for $\mathrm{C}_{36} \mathrm{H}_{27} \mathrm{~N}_{5} \mathrm{O}_{3} \mathrm{~S}: \mathrm{C}, 70.92 ; \mathrm{H}, 4.46 ; \overline{\mathrm{N}}, 11.49$. Found: C, 70.95; H, 4.52; N, 11.58 . 
2-[1-(Benzofuran-2-yl)ethylidenehydrazono]-5-[(1,3-Diphenyl-1H-pyrazol-4-yl)methylene]-3-(4chlorophenyl)thiazolidin-4-one

$5 d$

Yellow solid, yield 89\%, m.p. 242-4 ${ }^{\circ}$ C. FT-IR $\left(\mathrm{cm}^{-1}\right): 1714(\mathrm{C}=\mathrm{O}) ; 1609(\mathrm{C}=\mathrm{N}) ; 1230,1178,1081$ (C-O-C). ${ }^{1} \mathrm{H}-\mathrm{NMR}$ (DMSO-d $\mathrm{d}_{6}$, Mercury $\left.300 \mathrm{MHz}\right): \delta 2.27\left(\mathrm{~s}, 3 \mathrm{H},-\mathrm{N}=\mathrm{C}_{-} \mathrm{CH}_{3}\right) ; 7.25-7.84(\mathrm{~m}, 18 \mathrm{H}$, benzofuran $\mathrm{C}^{3,4,5,6,7}-\mathrm{Hs}, \mathrm{C}^{2,3,4,5,6}-\mathrm{Hs}$ of pyrazole $\mathrm{N}^{1}$-phenyl, $\mathrm{C}^{2,3,5,6}$ - $\mathrm{Hs}$ of $\mathrm{p}$-Cl-phenyl ring, $\mathrm{C}^{3,4,5}$ - $\mathrm{Hs}$ of pyrazole $\mathrm{C}^{3}$-phenyl and $-\mathrm{C}=\overline{\mathrm{C}} \mathrm{H}$ vinylic); $8.09\left(\mathrm{~d}, \mathrm{~J}=8 \mathrm{~Hz}, 2 \mathrm{H}, \mathrm{C}^{2,6}-\mathrm{Hs}\right.$ of pyrazole $\mathrm{C}^{3}$-phenyl); $8.96\left(\mathrm{~s}, \overline{\mathrm{H}}\right.$, pyrazole $\left.\mathrm{C}^{5}-\mathrm{H}\right)$. Anal. Calcd. for $\mathrm{C}_{35} \mathrm{H}_{24} \mathrm{ClN}_{5} \mathrm{O}_{2} \mathrm{~S}$ : C, 68.45; H, 3.94; N, 1 1.40. Found: C, 68.55; H, 3.98; N, 11.57 .

2-[1-(Benzofuran-2-yl)ethylidenehydrazono]-5-\{[3-(4-chlorophenyl)-1-phenyl-1H-pyrazol-4-yl]methylene $\}-3-(4-$ chlorophenyl)thiazolidin-4-one

$5 e$

Yellow solid, yield 79\%, m.p. 247-9 ${ }^{\circ}$ C. FT-IR $\left(\mathrm{cm}^{-1}\right): 1712(\mathrm{C}=\mathrm{O}) ; 1611(\mathrm{C}=\mathrm{N}) ; 1230,1180,1075(\mathrm{C}-\mathrm{O}-\mathrm{C})$. ${ }^{1} \mathrm{H}-\mathrm{NMR}$ (DMSO-d 6 , Mercury $300 \mathrm{MHz}$ ): $\delta 2.26$ (s, 3H, -N = C-CH 3 ); 7.26-7.37 (dist. dd, $1 \mathrm{H}$, benzofuran $\left.\mathrm{C}^{5}-\mathrm{H}\right) ; 7.37-7.52\left(\mathrm{~m}, 2 \mathrm{H}\right.$, benzofuran $\mathrm{C}^{6}-\mathrm{H}$ and $\mathrm{C}^{4}-\mathrm{H}$ of pyrazole $\mathrm{N}^{1}$-phenyl); 7.57-7.76 (m, 14H, benzofuran $\mathrm{C}^{3, \overline{4,7}-\mathrm{Hs}}, \mathrm{C}^{2,3,5,6}-\mathrm{Hs}$ of pyrazole $\mathrm{N}^{1}$-phenyl, $\mathrm{C}^{2,3,5,6}-\overline{\mathrm{H}}_{\mathrm{s}}$ of $\mathrm{p}$-Cl-phenyl ring of thiazolidinone, $\mathrm{C}^{3,5}-\mathrm{Hs}$ of $\mathrm{p}-\mathrm{Cl}-$ phenyl ring of pyrazole and $-\mathrm{C}=\mathrm{CH}$ vinylic); $8.08\left(\overline{\mathrm{d}}, \mathrm{J}=8 \mathrm{~Hz}, 2 \mathrm{H}, \mathrm{C}^{2,6}-\mathrm{Hs}\right.$ of p-Cl-phenyl ring of pyrazole); $8.96\left(\mathrm{~s}, 1 \mathrm{H}\right.$, pyrazole $\left.\mathrm{C}^{5}-\mathrm{H}\right)$. Anal. Calcd. for $\mathrm{C}_{35} \mathrm{H}_{23} \mathrm{Cl}_{2} \mathrm{~N}_{5} \mathrm{O}_{2} \mathrm{~S}: \mathrm{C}, 64.82 ; \overline{\mathrm{H}}, 3.57 ; \mathrm{N}, 10.80$. Found: $\mathrm{C}, 64.97$; $\mathrm{H}, 3.59 ; \mathrm{N}, 10.92$.

2-[1-(Benzofuran-2-yl)ethylidenehydrazono]-5-\{[3-(4-methoxyphenyl)-1-phenyl-1H-pyrazol-4-yl]methylene $\}-3-(4-$ chlorophenyl)thiazolidin-4-one

$5 f$

Yellow solid, yield 89\%, m.p. 226-8 ${ }^{\circ}$ C. FT-IR $\left(\mathrm{cm}^{-1}\right)$ : $1720(\mathrm{C}=\mathrm{O}) ; 1618(\mathrm{C}=\mathrm{N}) ; 1227,1183,1078(\mathrm{C}-\mathrm{O}-$ C). ${ }^{1} \mathrm{H}-\mathrm{NMR}\left(\mathrm{DMSO}_{6}\right.$, Mercury $\left.300 \mathrm{MHz}\right): \delta 2.26\left(\mathrm{~s}, 3 \mathrm{H},-\mathrm{N}=\mathrm{C}-\mathrm{CH}_{3}\right) ; 3.83\left(\mathrm{~s}, 3 \mathrm{H}, \mathrm{O}_{-} \mathrm{CH}_{3}\right) ; 7.13(\mathrm{~d}$, $\mathrm{J}=8.5 \mathrm{~Hz}, 2 \mathrm{H}, \mathrm{C}^{3,5}-\mathrm{Hs}$ of p-OCH 3 -phenyl ring); 7.31 (dist. dd, $1 \mathrm{H}, \mathrm{C}^{4}-\underline{\mathrm{H}}$ of pyrazole $\mathrm{N}^{1}$-phenyl); 7.39-7.47 $\left(\mathrm{m}, 2 \mathrm{H}\right.$, benzofuran $\left.\mathrm{C}^{\overline{5,6}}-\mathrm{Hs}\right) ; 7.55-7.66\left(\mathrm{~m}, 9 \mathrm{H}, \mathrm{C}^{2,3,5,6}-\mathrm{Hs}\right.$ of pyrazole $\mathrm{N}^{1}-$ phenyl, $\mathrm{C}^{2,6}-\mathrm{Hs}$ of p-Cl-phenyl ring, benzofuran $\mathrm{C}^{3,7}-\mathrm{Hs}$ and $-\overline{\mathrm{C}}=\mathrm{CH}$ vinylic); $7.69\left(\mathrm{~d}, \mathrm{~J}=8 . \overline{6} \mathrm{~Hz}, 2 \mathrm{H}, \mathrm{C}^{3,5}-\mathrm{Hs}\right.$ of p-Cl-phenyl ring); 7.71-7.78 (m, $1 \mathrm{H}$, benzofuran $\left.\overline{\mathrm{C}^{4}}-\underline{\mathrm{H}}\right) ; 8.07\left(\mathrm{~d}, \overline{\mathrm{J}}=8.5 \mathrm{~Hz}, 2 \mathrm{H}, \mathrm{C}^{2,6}-\mathrm{Hs}_{\text {s }}\right.$ of $\mathrm{p}-\mathrm{OCH}_{3}$-phenyl ring); $8.90\left(\mathrm{~s}, 1 \mathrm{H}\right.$, pyrazole $\left.\mathrm{C}^{5}-\underline{\mathrm{H}}\right)$. Anal. Calcd. for $\mathrm{C}_{36} \overline{\mathrm{H}}_{26} \mathrm{ClN}_{5} \mathrm{O}_{3} \mathrm{~S}: \mathrm{C}, 67.13 ; \mathrm{H}, 4.07 ; \overline{\mathrm{N}}, 10.87$. Found: C, 67.20; H, 4.11; N, 10.99 .

\section{2-[1-(Benzofuran-2-yl)ethylidenehydrazono]-5-[(1,3-Diphenyl-1H-pyrazol-4-yl)methylene]-3-(4- methoxyphenyl)thiazolidin-4-one \\ $5 g$}

Yellow solid, yield 81\%, m.p. 207-9 ${ }^{\circ}$ C. FT-IR $\left(\mathrm{cm}^{-1}\right)$ : $1719(\mathrm{C}=\mathrm{O}) ; 1597(\mathrm{C}=\mathrm{N}) ; 1234,1179,1079$ (C-OC). ${ }^{1} \mathrm{H}-\mathrm{NMR}\left(\mathrm{DMSO}_{\mathrm{d}}\right.$, Mercury $\left.300 \mathrm{MHz}\right): \delta 2.26\left(\mathrm{~s}, 3 \mathrm{H},-\mathrm{N}=\mathrm{C}^{-\mathrm{CH}_{3}}\right) ; 3.83\left(\mathrm{~s}, 3 \mathrm{H}, \mathrm{O}-\mathrm{CH}_{3}\right) ; 7.09(\mathrm{~d}$, $\mathrm{J}=9 \mathrm{~Hz}, 2 \mathrm{H}, \mathrm{C}^{3,5}-\mathrm{Hs}$ of p-OCH 3 -phenyl ring); 7.27-7.35 (dist. dd, $1 \mathrm{H}$, benzofuran $\mathrm{C}^{5}-\mathrm{H}$ ); 7.38-7.47 (m, $4 \mathrm{H}$, benzofuran $\mathrm{C}^{6}-\mathrm{H}, \mathrm{C}^{2,6}-\mathrm{Hs}$ of $\mathrm{p}-\mathrm{OCH}_{3}$-phenyl ring and $\mathrm{C}^{4}-\mathrm{H}$ of pyrazole $\mathrm{C}^{3}$-phenyl); $7.51-7.71(\mathrm{~m}, 10 \mathrm{H}$, benzofuran $\mathrm{C}^{3,7}-\mathrm{Hs}, \mathrm{C}^{2,3,4,5, \overline{6}}-\mathrm{H}$ s of pyrazole $\mathrm{N}^{1}$-phenyl, $\mathrm{C}^{3,5}-\mathrm{Hs}_{\mathrm{s}}$ of pyrazole $\mathrm{C}^{3}$-phenyl and $-\mathrm{C}=\mathrm{CHvinylic}$ ); $7.73\left(\mathrm{~d}, \mathrm{~J}=7.5 \overline{\mathrm{Hz}}, 1 \mathrm{H}\right.$, benzofuran $\left.\mathrm{C}^{4}-\mathrm{H}\right) ; 8.08\left(\mathrm{~d}, \mathrm{~J}=8 \overline{\mathrm{Hz}}, 2 \mathrm{H}, \mathrm{C}^{2,6}-\mathrm{Hs}\right.$ of pyrazole $\mathrm{C}^{3}$-phenyl); 8.93 (s, $1 \mathrm{H}$, pyrazole $\left.\mathrm{C}^{5}-\mathrm{H}\right)$. Anal. Calcd. for $\mathrm{C}_{36} \overline{\mathrm{H}}_{27} \mathrm{~N}_{5} \mathrm{O}_{3} \mathrm{~S} .1 / 2 \mathrm{H}_{2} \mathrm{O}$ : C, 69.89; $\mathrm{H}, 4.56 ; \mathrm{N}, 11.32 ; \mathrm{S}, 5.18$. Found: $\mathrm{C}$, 69.60; H, 4.42; N, 11.14; S, 4.82.

2-[1-(Benzofuran-2-yl)ethylidenehydrazono]-5-\{[3-(4-chlorophenyl)-1-phenyl-1H-pyrazol-4-yl]methylene $\}-3-(4-$ methoxyphenyl)thiazolidin-4-one

$5 h$

Yellow solid, yield 69\%, m.p. 280-2 ${ }^{\circ}$ C. FT-IR $\left(\mathrm{cm}^{-1}\right)$ : $1706(\mathrm{C}=\mathrm{O}) ; 1592(\mathrm{C}=\mathrm{N}) ; 1237,1185,1073(\mathrm{C}-\mathrm{O}-$ C). ${ }^{1} \mathrm{H}-\mathrm{NMR}\left(\mathrm{DMSO}_{6} \mathrm{~d}_{6}\right.$, Mercury $\left.300 \mathrm{MHz}\right): \delta 2.26\left(\mathrm{~s}, 3 \mathrm{H},-\mathrm{N}=\mathrm{C}^{-\mathrm{CH}_{3}}\right) ; 3.84\left(\mathrm{~s}, 3 \mathrm{H}, \mathrm{O}-\mathrm{CH}_{3}\right) ; 7.09$ (d, $\mathrm{J}=9 \mathrm{~Hz}, 2 \mathrm{H}, \mathrm{C}^{3,5}-\mathrm{Hs}$ of $\mathrm{p}-\mathrm{OCH}_{3}$-phenyl ring); 7.26-7.36 (dist. dd, $1 \mathrm{H}$, benzofuran $\mathrm{C}^{5}-\mathrm{H}$ ); 7.39-7.47 (m, $4 \mathrm{H}$, benzofuran $\mathrm{C}^{6}-\mathrm{H}, \mathrm{C}^{2,6}-\mathrm{Hs}$ of $\mathrm{p}-\mathrm{OCH}_{3}$-phenyl ring and $\mathrm{C}^{4}-\mathrm{H}$ of pyrazole $\mathrm{N}^{1}$-phenyl); 7.55-7.76 $(\mathrm{m}, 10 \mathrm{H}$, benzofuran $\mathrm{C}^{3,4,7}-\mathrm{Hs}, \mathrm{C}^{2,3,5, \overline{6}}-\mathrm{Hs}$ of pyrazole $\mathrm{N}^{1}$-phenyl, $\mathrm{C}^{3,5}-\mathrm{Hs}$ of $\mathrm{p}$-Cl-phenyl ring and $-\mathrm{C}=\mathrm{CH}$ vinylic); 8.07 (d, J $=8 \mathrm{~Hz}, 2 \mathrm{H}, \mathrm{C}^{2,6}-\mathrm{Hs}$ of p-Cl-phenyl ring); 8.95 (s, 1H, pyrazole $\left.\mathrm{C}^{5}-\mathrm{H}\right)$. EIMS m/z (\% abundance), 644 (76.12) $\mathrm{M}^{+}+1 ; 643(52.24) \mathrm{M}^{+} ; 189$ (100). Anal. Calcd. for $\mathrm{C}_{36} \mathrm{H}_{26} \mathrm{ClN}_{5} \mathrm{O}_{3} \mathrm{~S}: \mathrm{N}, 10.87 ; \mathrm{S}, 4.98$. Found: $\mathrm{N}$, 10.57; S, 5.03 . 
2-[1-(Benzofuran-2-yl)ethylidenehydrazono]-5-\{[3-(4-methoxyphenyl)-1-phenyl-1H-pyrazol-4-yl]methylene $\}-3-(4-$ methoxyphenyl)thiazolidin-4-one

5i

Yellow solid, yield 89\%, m.p. 282-4 ${ }^{\circ}$ C. FT-IR $\left(\mathrm{cm}^{-1}\right): 1702(\mathrm{C}=\mathrm{O}) ; 1596(\mathrm{C}=\mathrm{N}) ; 1241,1179,1071$ (C-O-C).

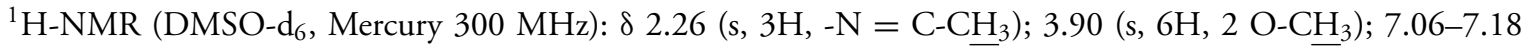

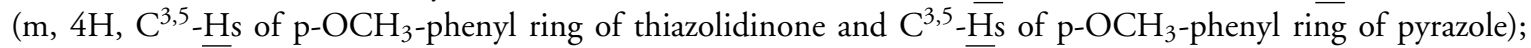
7.28-7.34 (dist. dd, $1 \mathrm{H}$, benzofuran $\mathrm{C}^{5}-\mathrm{H}$ ); 7.38-7.51 (m, 4H, benzofuran $\mathrm{C}^{6}-\mathrm{H}, \mathrm{C}^{2,6}-\mathrm{Hs}$ of $\mathrm{p}-\mathrm{OCH}_{3}$-phenyl ring of thiazolidinone \& $\mathrm{C}^{4}-\mathrm{H}$ of pyrazole $\mathrm{N}^{1}$-phenyl); 7.52-7.66 (m, $6 \mathrm{H}, \mathrm{C}^{2, \overline{3,5}, 6}-\mathrm{Hs}$ of pyrazole $\mathrm{N}^{1}$-phenyl, benzofuran $\mathrm{C}^{3}-\mathrm{H}$ and $-\mathrm{C}=\overline{\mathrm{CH}}$ vinylic); 7.67-7.76 (m, $2 \mathrm{H}$, benzofuran $\left.\mathrm{C}^{4,7}-\mathrm{Hs}\right) ; 8.07\left(\mathrm{~d}, \mathrm{~J}=7.5 \mathrm{~Hz}, 2 \mathrm{H}, \mathrm{C}^{2,6}\right.$ Hsof p-OCH $-\mathrm{OCH}_{3}$-phenyl ring of pyrazole); 8.90 (s, $1 \mathrm{H}$, pyrazole $\left.\mathrm{C}^{5}-\mathrm{H}\right)$. Anal. Calcd. for $\mathrm{C}_{37} \mathrm{H}_{29} \mathrm{~N}_{5} \mathrm{O}_{4} \mathrm{~S} \cdot 1 / 2 \mathrm{H}_{2} \mathrm{O}$ : $\overline{\mathrm{C}}$, 68.50; H, 4.66; N, 10.80; S, 4.94. Found: C, 68.82; H, 4.46; N, 10.79; S, 4.88.

2-[1-(Benzofuran-2-yl)ethylidenehydrazono]-5-[(1,3-Diphenyl-1H-pyrazol-4-yl)methylene]-3-cyclohexylthiazolidin-4one

$5 j$

Yellow solid, yield 56\%, m.p. 187-9 ${ }^{\circ}$ C. FT-IR $\left(\mathrm{cm}^{-1}\right): 1704(\mathrm{C}=\mathrm{O}) ; 1606(\mathrm{C}=\mathrm{N}) ; 1247,1177,1080(\mathrm{C}-$ O-C). ${ }^{1} \mathrm{H}-\mathrm{NMR}$ (DMSO-d ${ }_{6}$, Mercury $300 \mathrm{MHz}$ ): $\delta$ 1.08-1.49, 1.62-1.94 and 2.31-2.45 (3m, 10H, cyclohexyl $\left.\mathrm{C}^{2,3,4,5,6}-\mathrm{H}_{2}\right) ; 2.51$ (s, 3H, $\left.-\mathrm{N}=\mathrm{C}_{-} \mathrm{CH}_{3}\right) ; 4.43-4.57$ (m, $1 \mathrm{H}$, cyclohexyl $\left.\mathrm{C}^{1}-\mathrm{H}\right) ; 7.26-7.36$ (dist. dd, $1 \mathrm{H}$, benzofuran $\left.\mathrm{C}^{5}-\mathrm{H}\right) ; 7.39-7.46\left(\mathrm{~m}, 2 \mathrm{H}\right.$, benzofuran $\mathrm{C}^{6}-\mathrm{H}$ and $\mathrm{C}^{4}-\mathrm{H}$ of pyrazole $\mathrm{N}^{1}$-phenyl); 7.49-7.72 (m, 10H, benzofuran $\mathrm{C}^{3, \overline{7}}-\mathrm{Hs}, \mathrm{C}^{2,3,5,6}-\underline{\mathrm{H} s}$ of pyrazole $\mathrm{N}^{1}$-phenyl, $\mathrm{C}^{3,4,5}-\underline{\mathrm{H} s}$ of pyrazole $\mathrm{C}^{3}$-phenyl and $-\mathrm{C}=\mathrm{CH}$ vinylic); $7.74\left(\mathrm{~d}, \mathrm{~J}=7.5 \overline{\mathrm{Hz}}, 1 \mathrm{H}\right.$, benzofuran $\left.\mathrm{C}^{4}-\mathrm{H}\right) ; 8.05\left(\mathrm{~d}, \mathrm{~J}=8 \overline{\mathrm{Hz}}, 2 \mathrm{H}, \mathrm{C}^{2,6}-\mathrm{Hs}\right.$ of pyrazole $\mathrm{C}^{3}$-phenyl); 8.85 (s, $1 \mathrm{H}$, pyrazole $\left.\mathrm{C}^{5}-\underline{\mathrm{H}}\right)$. Anal. Calcd. for $\mathrm{C}_{35} \overline{\mathrm{H}_{31}} \mathrm{~N}_{5} \mathrm{O}_{2} \mathrm{~S}: \mathrm{C}, 71.77 ; \mathrm{H}, 5.33 ; \mathrm{N}, \overline{11}$.96. Found: C, 71.81; $\mathrm{H}, 5.35 ; \mathrm{N}$, 12.14 .

2-[1-(Benzofuran-2-yl)ethylidenehydrazono]-5-\{[3-(4-chlorophenyl)-1-phenyl-1H-pyrazol-4-yl]methylene \}-3cyclohexylthiazolidin-4-one

$5 k$

Yellow solid, yield 57\%, m.p. 210-2 ${ }^{\circ}$ C. FT-IR $\left(\mathrm{cm}^{-1}\right): 1711(\mathrm{C}=\mathrm{O}) ; 1608(\mathrm{C}=\mathrm{N}) ; 1247,1185,1079$ (C-O-C). ${ }^{1} \mathrm{H}-\mathrm{NMR}$ (DMSO-d 6 , Mercury $300 \mathrm{MHz}$ ): $\delta 1.08-1.47,1.61-1.99$ and $2.33-2.46\left(3 \mathrm{~m}, 10 \mathrm{H}\right.$, cyclohexyl C $\mathrm{C}^{2,3,4,5,6}$ $\left.\mathrm{H}_{2}\right) ; 2.52$ (s, 3H, $\left.-\mathrm{N}=\mathrm{C}_{-} \mathrm{CH}_{3}\right) ; 4.40-4.61$ (m, 1H, cyclohexyl $\left.\mathrm{C}^{1}-\underline{\mathrm{H}}\right) ; 7.29-7.35$ (dist. dd, $1 \mathrm{H}$, benzofuran $\mathrm{C}^{5}-\underline{\mathrm{H}}$ ); 7.39-7.47 (m, 2H, benzofuran $\mathrm{C}^{6}-\mathrm{H}$ and $\mathrm{C}^{4}-\mathrm{H}$ of pyrazole $\mathrm{N}^{1}$-phenyl); $7.52\left(\mathrm{~s}, 1 \mathrm{H}\right.$, benzofuran $\left.\mathrm{C}^{3}-\mathrm{H}\right) ; 7.57-7.73$ (m, 8H, benzofuran $\mathrm{C}^{7}-\underline{\mathrm{H}}, \mathrm{C}^{2,3,5,6}-\underline{\mathrm{H}} \mathrm{s}$ of pyrazole $\mathrm{N}^{1}$-phenyl, $\mathrm{C}^{3,5}-\underline{\mathrm{H}} \mathrm{s}$ of $\mathrm{p}-\mathrm{Cl}$-phenyl ring and $-\mathrm{C}=\mathrm{CH}$ vinylic); $7.74\left(\mathrm{~d}, \mathrm{~J}=7.5 \mathrm{~Hz}, 1 \overline{\mathrm{H}}\right.$, benzofuran $\left.\mathrm{C}^{4}-\mathrm{H}\right) ; 8.05\left(\mathrm{~d}, \mathrm{~J}=8 \mathrm{~Hz}, 2 \overline{\mathrm{H}}, \mathrm{C}^{2,6}-\mathrm{Hs}\right.$ of p-Cl-phenyl ring); $8 . \overline{88}(\mathrm{~s}, 1 \mathrm{H}$,

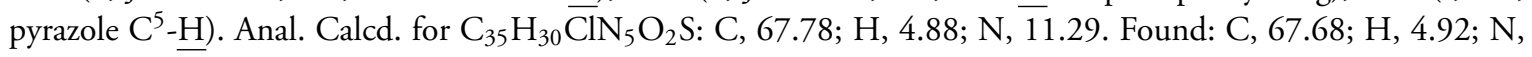
11.41 .

2-[1-(Benzofuran-2-yl)ethylidenehydrazono]-5-\{[3-(4-methoxyphenyl)-1-phenyl-1H-pyrazol-4-yl]methylene $\}-3-$ cyclohexylthiazolidin-4-one

$5 l$

Yellow solid, yield 93\%, m.p. $232-4^{\circ} \mathrm{C}$. FT-IR $\left(\mathrm{cm}^{-1}\right): 1712(\mathrm{C}=\mathrm{O}) ; 1601(\mathrm{C}=\mathrm{N})$; 1245, 1184, $1073(\mathrm{C}-$ O-C). ${ }^{1} \mathrm{H}-\mathrm{NMR}$ (DMSO-d $\mathrm{d}_{6}$, Mercury $\left.300 \mathrm{MHz}\right): \delta 1.15-1.49,1.69-1.99$ and $2.37-2.46(3 \mathrm{~m}, 10 \mathrm{H}$, cyclohexyl $\left.\mathrm{C}^{2,3,4,5,6}-\mathrm{H}_{2}\right) ; 2.54\left(\mathrm{~s}, 3 \mathrm{H},-\mathrm{N}=\mathrm{C}-\mathrm{CH}_{3}\right) ; 3.84\left(\mathrm{~s}, 3 \mathrm{H}, \mathrm{O}-\mathrm{CH}_{3}\right) ; 4.46-4.61\left(\mathrm{~m}, 1 \mathrm{H}\right.$, cyclohexyl $\left.\mathrm{C}^{1}-\mathrm{H}\right) ; 7.12$ $\left(\mathrm{d}, \mathrm{J}=8.5 \mathrm{~Hz}, 2 \mathrm{H}, \mathrm{C}^{3,5}-\mathrm{Hs}\right.$ of $\mathrm{p}-\mathrm{OCH}_{3}$-phenyl ring); $7.31-7.37$ (dist. dd, $1 \mathrm{H}$, benzofuran $\mathrm{C}^{5}-\mathrm{H}$ ); $7.38-7.43$ (dist. dd, $1 \mathrm{H}$, benzofuran $\overline{\mathrm{C}}^{6}-\mathrm{H}$ ); 7.44-7.50 (dist. dd, $1 \mathrm{H}, \mathrm{C}^{4}-\mathrm{H}$ of pyrazole $\mathrm{N}^{1}$-phenyl); $7.52(\mathrm{~s}, 1 \overline{\mathrm{H}}$, benzofuran $\left.\mathrm{C}^{3}-\underline{\mathrm{H}}\right) ; 7.53-7.60\left(\mathrm{~m}, 4 \mathrm{H}, \overline{\mathrm{C}^{2,3}, 5,6}-\underline{\mathrm{H} s}\right.$ of pyrazole $\mathrm{N}^{1}$-phenyl); $7.66\left(\mathrm{~d}, \mathrm{~J}=8 \mathrm{~Hz}, 1 \mathrm{H}\right.$, benzofuran $\left.\mathrm{C}^{7}-\mathrm{H}\right) ; 7.74$ $\left(\mathrm{d}, \overline{\mathrm{J}}=8 \mathrm{~Hz}, 1 \mathrm{H}\right.$, benzofuran $\left.\mathrm{C}^{4}-\underline{\mathrm{H}}\right) ; 7.91\left(\mathrm{~s}, 1 \mathrm{H},-\mathrm{C}=\mathrm{C} \mathrm{H}\right.$ vinylic); $8.02\left(\mathrm{~d}, \mathrm{~J}=8.3 \mathrm{~Hz}, 2 \mathrm{H}, \mathrm{C}^{\overline{2,6}}-\mathrm{Hs}\right.$ of p- $\mathrm{OCH}_{3}$-phenyl ring); $8.76\left(\mathrm{~s}, 1 \mathrm{H}\right.$, pyrazole $\left.\mathrm{C}^{5}-\underline{\mathrm{H}}\right)$. Anal. Calcd. for $\mathrm{C}_{36} \mathrm{H}_{33} \mathrm{~N}_{5} \mathrm{O}_{3} \mathrm{~S}: \mathrm{C}, 70.22 ; \mathrm{H}, 5.40 ; \mathrm{N}, \overline{11} .37$. Found: C, 70.35; H, 5.46; N, 11.49 .

2-[1-(1H-Benzimidazole-2-yl)ethylidenehydrazono]-5-[(1,3-Diphenyl-1H-pyrazol-4-yl)methylene]-3phenylthiazolidin-4-one

$5 m$

Yellow solid, yield 55\%, m.p. $>300^{\circ} \mathrm{C}$. FT-IR $\left(\mathrm{cm}^{-1}\right): 3333(\mathrm{~N}-\mathrm{H}) ; 1718(\mathrm{C}=\mathrm{O}) ; 1623(\mathrm{C}=\mathrm{N}) .{ }^{1} \mathrm{H}-\mathrm{NMR}$ (DMSO-d ${ }_{6}$, Jeol $\left.500 \mathrm{MHz}\right): \delta 2.49\left(\mathrm{~s}, 3 \mathrm{H},-\mathrm{N}=\mathrm{C}^{-\mathrm{CH}_{3}}\right) ; 7.08-7.16\left(\mathrm{~m}, 1 \mathrm{H}, \mathrm{C}^{4}-\mathrm{H}\right.$ of thiazolidinone $\mathrm{N}^{3}$-phenyl); 7.18-7.25 (dist. dd, $1 \mathrm{H}$, benzimidazole- $\mathrm{C}^{5}-\underline{\mathrm{H}}$ ); 7.26-7.33 (dist. dd, $1 \mathrm{H}$, benzimidazole- $\mathrm{C}^{6}-\underline{\mathrm{H}}$ ); 7.40-7.75 (m, 
$15 \mathrm{H}$, benzimidazole $\mathrm{C}^{4,7}-\mathrm{Hs}, \mathrm{C}^{2,3,5,6}-\mathrm{Hs}$ of thiazolidinone $\mathrm{N}^{3}$-phenyl, $\mathrm{C}^{3,4,5}-\mathrm{Hs}$ of pyrazole $\mathrm{C}^{3}$-phenyl, $\mathrm{C}^{2,3,4,5,6}$ Hs of pyrazole $\mathrm{N}^{1}$-phenyl and $-\mathrm{C}=\overline{\mathrm{CH}}$ vinylic); 8.08 (d, J $=8 \mathrm{~Hz}, 2 \mathrm{H}, \mathrm{C}^{2,6}-\overline{\mathrm{H}}$ s of pyrazole $\mathrm{C}^{3}$-phenyl); 8.97 (s, $\overline{1 \mathrm{H}}$, pyrazole $\left.\mathrm{C}^{5}-\mathrm{H}\right) ; 11.21$ (s, $1 \mathrm{H},-\mathrm{NH}$ of benzimidazole, $\mathrm{D}_{2} \mathrm{O}$ exchangeable). Anal. Calcd. for $\mathrm{C}_{34} \mathrm{H}_{25} \mathrm{~N}_{7} \mathrm{OS}$ : C, $70.45 ; \mathrm{H}, 4.35 ; \mathrm{N}, 16.91$. Found: $\mathrm{C}, 70.49 ; \mathrm{H}, 4.42 ; \mathrm{N}, 17.04$.

2-[1-(1H-Benzimidazole-2-yl)ethylidenehydrazono]-5-\{[3-(4-chlorophenyl)-1-phenyl-1H-pyrazol-4-yl]methylene $\}-3-$ phenylthiazolidin-4-one

$5 n$

Yellow solid, yield 57\%, m.p. $>300^{\circ}$ C. FT-IR $\left(\mathrm{cm}^{-1}\right): 3325(\mathrm{~N}-\mathrm{H}) ; 1715(\mathrm{C}=\mathrm{O}) ; 1620(\mathrm{C}=\mathrm{N}) .{ }^{1} \mathrm{H}-\mathrm{NMR}$ (DMSO-d $\mathrm{d}_{6}$, Mercury $\left.300 \mathrm{MHz}\right): \delta 2.57\left(\mathrm{~s}, 3 \mathrm{H},-\mathrm{N}=\mathrm{C}-\mathrm{CH}_{3}\right) ; 7.10-7.17\left(\mathrm{~m}, 1 \mathrm{H}, \mathrm{C}^{4}-\mathrm{H}\right.$ of thiazolidinone $\mathrm{N}^{3}-$ phenyl); 7.20-7.34 (m, 2H, benzimidazole- $\left.\mathrm{C}^{5,6}-\mathrm{Hs}\right) ; 7.45$ (dist. dd, $1 \mathrm{H}, \mathrm{C}^{4}-\mathrm{H}$ of pyrazole $\mathrm{N}^{1}$-phenyl); 7.56-7.81 $\left(\mathrm{m}, 13 \mathrm{H}, \mathrm{C}^{2,3,5,6}-\mathrm{H}\right.$ s of thiazolidinone $\mathrm{N}^{3}$-phenyl, $-\mathrm{C}=\mathrm{CH}$ vinylic, benzimidazole $\mathrm{C}^{4,7}-\underline{\mathrm{H}} \mathrm{s}, \mathrm{C}^{2,3,5,6}-\mathrm{H}$ s of pyrazole $\mathrm{N}^{1}$-phenyl and $\mathrm{C}^{\overline{3,5}}-\underline{\mathrm{H}}$ s of p-Cl-phenyl ring); $8.08\left(\mathrm{~d}, \mathrm{~J}=\overline{7} .5 \mathrm{~Hz}, 2 \mathrm{H}, \mathrm{C}^{2,6}-\underline{\mathrm{H} s}\right.$ of p-Cl-phenyl ring); 8.94 (s, $1 \mathrm{H}$,

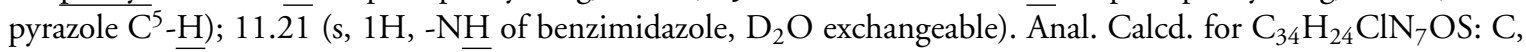
66.50; H, 3.94; N, 15.97. Found: $\overline{\mathrm{C}}, 66.48 ; \mathrm{H}, 3.98 ; \mathrm{N}, 16.13$.

2-[1-(1H-Benzimidazole-2-yl)ethylidenehydrazono]-5-\{[3-(4-methoxyphenyl)-1-phenyl-1H-pyrazol-4-yl]methylene $\}$ 3-phenylthiazolidin-4-one

50

Yellow solid, yield 56\%, m.p. $>300^{\circ}$ C. FT-IR $\left(\mathrm{cm}^{-1}\right): 3319(\mathrm{~N}-\mathrm{H}) ; 1719(\mathrm{C}=\mathrm{O}) ; 1617(\mathrm{C}=\mathrm{N})$. EIMS m/z (\% abundance), $611(14.83) \mathrm{M}^{+}+2 ; 610(42.72) \mathrm{M}^{+}+1 ; 609(100) \mathrm{M}^{+}$. Anal. Calcd. for $\mathrm{C}_{35} \mathrm{H}_{27} \mathrm{~N}_{7} \mathrm{O}_{2} \mathrm{~S}: \mathrm{C}$, 68.95; H, 4.46; N, 16.08. Found: C, 69.07; H, 4.53; N, 16.22 .

2-[1-(1H-Benzimidazole-2-yl)ethylidenehydrazono]-5-[(1,3-Diphenyl-1H-pyrazol-4-yl)methylene]-3-(4chlorophenyl)thiazolidin-4-one

$5 p$

Yellow solid, yield 72\%, m.p. 286-8. FT-IR $\left(\mathrm{cm}^{-1}\right): 3319(\mathrm{~N}-\mathrm{H}) ; 1723(\mathrm{C}=\mathrm{O}) ; 1598(\mathrm{C}=\mathrm{N}) .{ }^{1} \mathrm{H}-\mathrm{NMR}$ (DMSO-d $\mathrm{d}_{6}$, Jeol $500 \mathrm{MHz}$ ): $\delta 2.33$ (s, 3H, -N = C- $\mathrm{CH}_{3}$ ); 7.18-7.23 (dist. dd, $1 \mathrm{H}$, benzimidazole $\mathrm{C}^{5}-\mathrm{H}$ ); $7.28-$ 7.33 (dist. dd, $1 \mathrm{H}$, benzimidazole $\left.\mathrm{C}^{6}-\mathrm{H}\right) ; 7.45\left(\mathrm{t}, \mathrm{J}=\overline{7} .5 \mathrm{~Hz}, 1 \mathrm{H}, \mathrm{C}^{4}-\mathrm{H}\right.$ of pyrazole $\mathrm{C}^{3}$-phenyl); $7.49-7.71(\mathrm{~m}$, $13 \mathrm{H}$, benzimidazole $\mathrm{C}^{4,7}-\mathrm{Hs}, \mathrm{C}^{2,3,5,6}-\overline{\mathrm{H}} \mathrm{s}$ of $\mathrm{p}$-Cl-phenyl ring, $\mathrm{C}^{2,3,4,5,6}-\overline{\mathrm{H}} \mathrm{s}$ of pyrazole $\mathrm{N}^{1}$-phenyl and $\mathrm{C}^{3,5}-\mathrm{H} s$ of pyrazole $\mathrm{C}^{3}$-phenyl); $7.77 \overline{(\mathrm{s}}, 1 \mathrm{H},-\mathrm{C}=\mathrm{CH}$ vinylic); $8.06\left(\mathrm{~d}, \mathrm{~J}=8 \mathrm{~Hz}, \overline{2 \mathrm{H}}, \mathrm{C}^{2,6}-\mathrm{H}_{\mathrm{H}}\right.$ of pyrazole $\mathrm{C}^{3}$-phenyl); 8.86 $\left(\mathrm{s}, 1 \mathrm{H}\right.$, pyrazole $\left.\mathrm{C}^{5}-\mathrm{H}\right) ; 12.6$ (s, $1 \mathrm{H},-\mathrm{NH}$ of benzimidazole, $\mathrm{D}_{2} \mathrm{O}$ exchangeable). Anal. Calcd. for $\mathrm{C}_{34} \overline{\mathrm{H}}_{24} \mathrm{ClN}_{7} \mathrm{OS}$ : C, 66.50; H, 3.94; $\overline{\mathrm{N}}, 15.97$. Found: $\mathrm{C}, 66.59 ; \mathrm{H}, 4.02 ; \mathrm{N}, 16.13$.

2-[1-(1H-Benzimidazole-2-yl)ethylidenehydrazono]-5-\{[3-(4-chlorophenyl)-1-phenyl-1H-pyrazol-4-yl]methylene $\}-3-$ (4-chlorophenyl)thiazolidin-4-one

$5 q$

Yellow solid, yield 60\%, m.p. >300. FT-IR $\left(\mathrm{cm}^{-1}\right): 3335(\mathrm{~N}-\mathrm{H}) ; 1715(\mathrm{C}=\mathrm{O}) ; 1603(\mathrm{C}=\mathrm{N}) .{ }^{1} \mathrm{H}-\mathrm{NMR}$ (DMSO-d $\mathrm{d}_{6}$, Mercury $\left.300 \mathrm{MHz}\right): \delta 2.37,2.58\left(2 \mathrm{~s}, 3 \mathrm{H},-\mathrm{N}=\mathrm{C}^{-\mathrm{CH}_{3}}\right.$ due to isomerism); 7.10-7.55 (m, $2 \mathrm{H}$, benzimidazole $\mathrm{C}^{5,6}-\mathrm{H}$ due to isomerism); 7.56-7.95 (m, $14 \mathrm{H}, \mathrm{C}^{4,7}-\mathrm{H}$ of benzimidazole, $-\mathrm{C}=\mathrm{CH}$ vinylic, $\mathrm{C}^{2,3,4,5,6}$ - $\mathrm{Hs}$ of pyrazole $\mathrm{N}^{1}$-phenyl, $\mathrm{C}^{2,3,5,6}-\mathrm{Hs}$ of $\mathrm{p}$-Cl-phenyl ring of thiazolidinone and $\mathrm{C}^{3,5}$ - $\mathrm{Hs}$ of $\mathrm{p}$ - $\overline{\mathrm{Cl}}$-phenyl ring of pyrazole); 8.04-8.13 (m, $2 \mathrm{H}, \mathrm{C}^{2,6}-\overline{\mathrm{Hs}}$ of $\mathrm{p}$-Cl-phenyl ring of pyrazole due to isomerism); $8.89,8.96$ (2s, $1 \mathrm{H}$, pyrazole $\mathrm{C}^{5}-\mathrm{H}$ due to isomerism); $11 . \overline{27}, 12.56\left(2 \mathrm{~s}, 1 \mathrm{H},-\mathrm{NH}\right.$ of benzimidazole, $\mathrm{D}_{2} \mathrm{O}$ exchangeable due to isomerism). Anal. Calcd. for $\mathrm{C}_{34} \mathrm{H}_{23} \mathrm{Cl}_{2} \mathrm{~N}_{7} \mathrm{OS}$ : C, 62.96; H, 3.57; $\overline{\mathrm{N}}, 15.12$. Found: C, 63.09; H, 3.54; N, 15.27.

2-[1-(1H-Benzimidazole-2-yl)ethylidenehydrazono]-5-\{[3-(4-methoxyphenyl)-1-phenyl-1H-pyrazol-4-yl]methylene $\}$ 3-(4-chlorophenyl)thiazolidin-4-one

$5 r$

Yellow solid, yield 69\%, m.p. >300. FT-IR $\left(\mathrm{cm}^{-1}\right): 3340(\mathrm{~N}-\mathrm{H}) ; 1707(\mathrm{C}=\mathrm{O}) ; 1608(\mathrm{C}=\mathrm{N}) .{ }^{1} \mathrm{H}-\mathrm{NMR}$ (DMSO$\mathrm{d}_{6}$, Mercury $\left.300 \mathrm{MHz}\right): \delta 2.37\left(\mathrm{~s}, 3 \mathrm{H},-\mathrm{N}=\mathrm{C}-\mathrm{CH}_{3}\right) ; 3.84\left(\mathrm{~s}, 3 \mathrm{H}, \mathrm{O}-\mathrm{CH}_{3}\right) ; 7.14\left(\mathrm{~d}, \mathrm{~J}=8.5 \mathrm{~Hz}, 2 \mathrm{H}, \mathrm{C}^{3,5}-\underline{\mathrm{H}} \mathrm{s}\right.$ of p- $\mathrm{OCH}_{3}$-phenyl ring); 7.20-7.28 (dist. dd, $1 \mathrm{H}$, benzimidazole $\mathrm{C}^{5}-\mathrm{H}$ ); $7.30-7.38$ (dist. dd, $1 \mathrm{H}$, benzimidazole $\left.\mathrm{C}^{6}-\mathrm{H}\right) ; 7.46-7.50$ (dist. t, $1 \mathrm{H}, \mathrm{C}^{4}-\mathrm{H}$ of pyrazole $\mathrm{N}^{1}$-phenyl); 7.61-7.68 $\left(\mathrm{m}, 9 \mathrm{H}\right.$, benzimidazole $\mathrm{C}^{4}-\mathrm{H}, \mathrm{C}^{2,3,5,6}-\mathrm{Hs}$

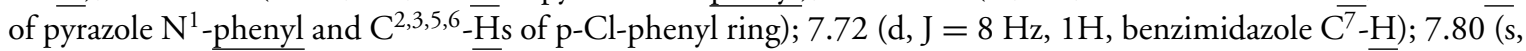
$1 \mathrm{H},-\mathrm{C}=\mathrm{CH}$ vinylic); $8.07\left(\mathrm{~d}, \mathrm{~J}=8.5 \mathrm{~Hz}, 2 \mathrm{H}, \mathrm{C}^{2,6}-\underline{\mathrm{Hs}}\right.$ of $\mathrm{p}-\mathrm{OCH}_{3}$-phenyl ring); 8.94 (s, $1 \mathrm{H}$, pyrazole $\left.\mathrm{C}^{5}-\underline{\mathrm{H}}\right)$; 12.61 (s, $1 \mathrm{H},-\mathrm{NH}$ of benzimidazole, $\mathrm{D}_{2} \mathrm{O}$ exchangeable). Anal. Calcd. for $\mathrm{C}_{35} \mathrm{H}_{26} \mathrm{ClN}_{7} \mathrm{O}_{2} \mathrm{~S} .1 / 2 \mathrm{H}_{2} \mathrm{O}: \mathrm{N}, 15 . \overline{0} 1$; S, 4.91. Found: $\bar{N}, 14.67 ;$ S, 5.23 . 
2-[1-(1H-Benzimidazole-2-yl)ethylidenehydrazono]-5-[(1,3-Diphenyl-1H-pyrazol-4-yl)methylene]-3-(4methoxyphenyl)thiazolidin-4-one

$5 s$

Yellow solid, yield 72\%, m.p. >300. FT-IR $\left(\mathrm{cm}^{-1}\right): 3342(\mathrm{~N}-\mathrm{H}) ; 1715(\mathrm{C}=\mathrm{O}) ; 1613(\mathrm{C}=\mathrm{N}) .{ }^{1} \mathrm{H}-\mathrm{NMR}$ (DMSO-d ${ }_{6}$, Mercury $\left.300 \mathrm{MHz}\right): \delta 2.57\left(\mathrm{~s}, 3 \mathrm{H},-\mathrm{N}=\mathrm{C}_{-} \mathrm{CH}_{3}\right) ; 3.92\left(\mathrm{~s}, 3 \mathrm{H},-\mathrm{OCH}_{3}\right) ; 7.02-7.52\left(\mathrm{~m}, 5 \mathrm{H}, \mathrm{C}^{3,5}-\mathrm{Hs}\right.$ of p-OCH 3 -phenyl ring, benzimidazole $\mathrm{C}^{5,6}-\underline{\mathrm{H}}$ and $\mathrm{C}^{4}-\mathrm{H}$ of pyrazole $\mathrm{C}^{3}$-phenyl); $7.53-7.77\left(\mathrm{~m}, 12 \mathrm{H}, \mathrm{C}^{2,6}-\mathrm{Hs}\right.$ of p-OCH 3 -phenyl ring, benzimidazole $\mathrm{C}^{4,7}-\underline{\mathrm{H}},-\mathrm{C}=\mathrm{CH}$ vinylic, $\mathrm{C}^{2,3,4,5,6}-\underline{\mathrm{Hs}}$ of pyrazole $\mathrm{N}^{1}$-phenyl and $\mathrm{C}^{3,5}-\underline{\mathrm{H} s}$ of pyrazole $\mathrm{C}^{3}$-phenyl); 8.06-8.14 (dist. d, $\overline{2} \mathrm{H}, \mathrm{C}^{2,6}-\overline{\mathrm{Hs}}$ of pyrazole $\mathrm{C}^{3}$-phenyl); 8.95 (br. s, $1 \mathrm{H}$, pyrazole $\mathrm{C}^{5}-\overline{\mathrm{H}}$ ); 11.27 (s, $1 \mathrm{H}$, - $\mathrm{NH}$ of benzimidazole, $\mathrm{D}_{2} \mathrm{O}$ exchangeable). Anal. Calcd. for $\mathrm{C}_{35} \mathrm{H}_{27} \mathrm{~N}_{7} \mathrm{O}_{2} \mathrm{~S}: \mathrm{C}, 68.95 ; \mathrm{H}, 4.46 ; \overline{\mathrm{N}}$, 16.08; S, 5.26. Found: C, 68.74; H, 4.29; N, 16.07; S, 5.51.

2-[1-(1H-Benzimidazole-2-yl)ethylidenehydrazono]-5-\{[3-(4-chlorophenyl)-1-phenyl-1H-pyrazol-4-yl]methylene $\}-3-$ (4-methoxyphenyl)thiazolidin-4-one

$5 t$

Yellow solid, yield 72\%, m.p. >300. FT-IR $\left(\mathrm{cm}^{-1}\right)$ : $3327(\mathrm{~N}-\mathrm{H}) ; 1707(\mathrm{C}=\mathrm{O}) ; 1597(\mathrm{C}=\mathrm{N}) .{ }^{1} \mathrm{H}-\mathrm{NMR}$ (DMSO-d $\mathrm{d}_{6}$, Mercury $\left.300 \mathrm{MHz}\right): \delta 2.37\left(\mathrm{~s}, 3 \mathrm{H},-\mathrm{N}=\mathrm{C}_{-} \mathrm{CH}_{3}\right) ; 3.93\left(\mathrm{~s}, 3 \mathrm{H},-\mathrm{OCH}_{3}\right) ; 7.06-7.78(\mathrm{~m}, 16 \mathrm{H}$, benzimidazole $\mathrm{C}^{4,5,6,7}-\mathrm{Hs}, \mathrm{C}^{2,3,5,6}-\mathrm{Hs}$ of $\mathrm{p}-\mathrm{OCH}_{3}$-phenyl ring, $\mathrm{C}^{2,3,4,5,6}-\mathrm{Hs}$ of pyrazole $\mathrm{N}^{1}$-phenyl, $\mathrm{C}^{3,5}-\underline{\mathrm{H}}$ of p-Cl-phenyl ring and $\overline{-\mathrm{C}}=\mathrm{CH}$ vinylic); 8.06-8.13 (dist. d, $2 \mathrm{H}, \mathrm{C}^{2,6}-\mathrm{Hs}$ of p-Cl-phenyl ring); 8.99 (br. s, $1 \mathrm{H}$, pyrazole $\left.\mathrm{C}^{5}-\underline{\mathrm{H}}\right) ; 11.28\left(\mathrm{~s}, 1 \mathrm{H},-\overline{\mathrm{NH}}\right.$ of benzimidazole, $\mathrm{D}_{2} \mathrm{O}$ exchangeable). Anal. Calcd. for $\mathrm{C}_{35} \mathrm{H}_{26} \mathrm{ClN}_{7} \mathrm{O}_{2} \mathrm{~S}: \mathrm{C}$, 65.26; H, 4. $\overline{07} ; \mathrm{N}, 15.22$. Found: $\bar{C}, 65.41 ; \mathrm{H}, 4.13 ; \mathrm{N}, 15.43$.

2-[1-(1H-Benzimidazole-2-yl)ethylidenehydrazono]-5-\{[3-(4-methoxyphenyl)-1-phenyl-1H-pyrazol-4-yl]methylene $\}$ 3-(4-methoxyphenyl)thiazolidin-4-one

$5 u$

Yellow solid, yield 79\%, m.p. >300. FT-IR $\left(\mathrm{cm}^{-1}\right): 3341(\mathrm{~N}-\mathrm{H}) ; 1712(\mathrm{C}=\mathrm{O}) ; 1600(\mathrm{C}=\mathrm{N}) .{ }^{1} \mathrm{H}-\mathrm{NMR}$ (DMSO$\mathrm{d}_{6}$, Jeol $\left.500 \mathrm{MHz}\right): \delta 2.55\left(\mathrm{~s}, 3 \mathrm{H},-\mathrm{N}=\mathrm{C}-\mathrm{CH}_{3}\right) ; 3.81$ (s, 3H, O- $\mathrm{CH}_{3}$ of p-OCH -phenyl of thiazolidinone); 3.90 $\left(\mathrm{s}, 3 \mathrm{H}, \mathrm{O}-\mathrm{CH}_{3}\right.$ of $\mathrm{p}-\mathrm{OCH}_{3}$-phenyl ring of pyrazole); 7.00-7.16 (dist. $2 \mathrm{~d}, 4 \mathrm{H}, \mathrm{C}^{3,5}-\mathrm{Hs}$ of $\mathrm{p}-\mathrm{OCH}_{3}$-phenyl ring of

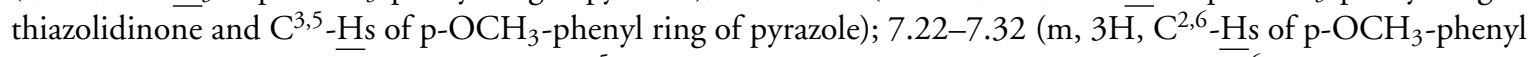
ring of thiazolidinone and benzimidazole $\left.\mathrm{C}^{5}-\mathrm{H}\right)$; 7.37-7.44 (dist. dd, $1 \mathrm{H}$, benzimidazole $\left.\overline{\mathrm{C}}^{6}-\mathrm{H}\right)$; 7.54-7.72 (m, $8 \mathrm{H}, \mathrm{C}^{3,4,5}-\mathrm{Hs}$ of pyrazole $\mathrm{N}^{1}$-phenyl, $\mathrm{C}^{4,7}-\underline{\mathrm{H}}$ of benzimidazole, $\mathrm{C}^{2,6}-\mathrm{Hs}$ of $\mathrm{p}-\mathrm{OCH}_{3}$-phenyl ring of pyrazole, $-\mathrm{C}=\mathrm{CH}$ vinylic); 8.00-8.07 (m, 2H, $\mathrm{C}^{2,6}-\overline{\mathrm{Hs}}$ of pyrazole $\mathrm{N}^{1}$-phenyl); 8.98 (br. s, $1 \mathrm{H}$, pyrazole $\left.\mathrm{C}^{5}-\mathrm{H}\right) ; 11.25$ (s, $1 \mathrm{H},-\mathrm{N} \overline{\mathrm{H}}$ of benzimidazole, $\mathrm{D}_{2} \mathrm{O}$ exchangeable). Anal. Calcd. for $\mathrm{C}_{36} \mathrm{H}_{29} \mathrm{~N}_{7} \mathrm{O}_{3} \mathrm{~S} .1 / 2 \mathrm{H}_{2} \mathrm{O}: \mathrm{C}, 66.65 ; \overline{\mathrm{H}}, 4.66 ; \mathrm{N}$, 15.11; S, 4.94. Found: C, 66.49; H, 4.63; N, 15.02; S, 5.02 .

\section{2-[1-(1H-Benzimidazole-2-yl)ethylidenehydrazono]-5-[(1,3-Diphenyl-1H-pyrazol-4-yl)methylene]-3- cyclohexylthiazolidin-4-one \\ $5 v$}

Yellow solid, yield 71\%, m.p. 230-2. FT-IR $\left(\mathrm{cm}^{-1}\right): 3320(\mathrm{~N}-\mathrm{H}) ; 1700(\mathrm{C}=\mathrm{O}) ; 1610(\mathrm{C}=\mathrm{N}) .{ }^{1} \mathrm{H}-\mathrm{NMR}$ (DMSO-d $\mathrm{d}_{6}$, Jeol $\left.500 \mathrm{MHz}\right): \delta 1.03-1.40,1.61-1.86$ and $2.35-2.42\left(3 \mathrm{~m}, 10 \mathrm{H}\right.$, cyclohexyl $\left.\mathrm{C}^{2,3,4,5,6}-\mathrm{H}_{2}\right) ; 2.59$ $\left(\mathrm{s}, 3 \mathrm{H},-\mathrm{N}=\mathrm{C}_{-} \mathrm{CH}_{3}\right)$; 4.43-4.51 (m, $1 \mathrm{H}$, cyclohexyl $\left.\mathrm{C}^{1}-\mathrm{H}\right)$; 7.18-7.24 (dist. dd, $1 \mathrm{H}$, benzimidazole $\left.\mathrm{C}^{5}-\mathrm{H}\right)$;

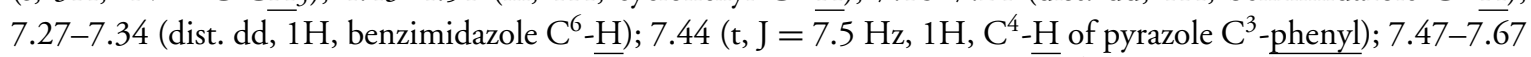
$\left(\mathrm{m}, 9 \mathrm{H},-\mathrm{C}=\mathrm{CH}\right.$ vinylic, $\mathrm{C}^{3,5}-\mathrm{Hs}$ of pyrazole $\mathrm{C}^{3}$-phenyl, benzimidazole $\mathrm{C}^{4}-\mathrm{H}$ and $\mathrm{C}^{2,3,4,5,6}-\mathrm{Hs}$ of pyrazole $\mathrm{N}^{1}$-phenyl); $7.70 \overline{(\mathrm{d}}, \mathrm{J}=8 \mathrm{~Hz}, \overline{1 \mathrm{H}}$, benzimidazole $\left.\mathrm{C}^{7}-\mathrm{H}\right) ; 8.02\left(\mathrm{~d}, \mathrm{~J}=8 \mathrm{~Hz}, 2 \mathrm{H}, \mathrm{C}^{2,6}-\mathrm{Hs}\right.$ of pyrazole $\mathrm{C}^{3}-$ phenyl); 8.79 (s, $1 \mathrm{H}$, pyrazole $\left.\mathrm{C}^{5}-\underline{\mathrm{H}}\right) ; 12.56\left(\mathrm{~s}, 1 \mathrm{H},-\mathrm{NH}\right.$ of benzimidazole, $\mathrm{D}_{2} \mathrm{O}$ exchangeable) . Anal. Calcd. for $\overline{\mathrm{C}}_{34} \mathrm{H}_{31} \mathrm{~N}_{7} \mathrm{OS} .1 / 2 \mathrm{H}_{2} \mathrm{O}$ : C, 68.66; H, 5.42; N, 16.49; S, 5.39. Found: C, 68.21; H, 5.34; N, 16.16; S, 5.52.

2-[1-(1H-Benzimidazole-2-yl)ethylidenehydrazono]-5-\{[3-(4-chlorophenyl)-1-phenyl-1H-pyrazol-4-yl]methylene $\}-3-$ cyclohexylthiazolidin-4-one

$5 w$

Yellow solid, yield 82\%, m.p. 167-9. FT-IR $\left(\mathrm{cm}^{-1}\right): 3336(\mathrm{~N}-\mathrm{H}) ; 1714(\mathrm{C}=\mathrm{O}) ; 1611(\mathrm{C}=\mathrm{N}) \cdot{ }^{1} \mathrm{H}-\mathrm{NMR}$ (DMSO-d 6 , Mercury $300 \mathrm{MHz}$ ): $\delta 1.11-1.48,1.64-1.90$ and 2.32-2.49 $\left(3 \mathrm{~m}, 10 \mathrm{H}\right.$, cyclohexyl $\left.\mathrm{C}^{2,3,4,5,6}-\mathrm{H}_{2}\right)$; $2.61\left(\mathrm{~s}, 3 \mathrm{H},-\mathrm{N}=\mathrm{C}-\mathrm{CH}_{3}\right) ; 4.47-4.56\left(\mathrm{~m}, 1 \mathrm{H}\right.$, cyclohexyl $\left.\mathrm{C}^{1}-\mathrm{H}\right) ; 7.20-7.28$ (dist. dd, $1 \mathrm{H}$, benzimidazole $\left.\mathrm{C}^{5}-\mathrm{H}\right)$; 7.30-7.37 (dist. dd, 1 H, benzimidazole $\left.\mathrm{C}^{6}-\underline{\mathrm{H}}\right) ; 7.47\left(\mathrm{t}, \mathrm{J}=7.5 \overline{\mathrm{Hz}}, 1 \mathrm{H}, \mathrm{C}^{4}-\underline{\mathrm{H}}\right.$ of pyrazole $\mathrm{N}^{1}$-phenyl); 7.52 (s, $\overline{1 \mathrm{H}}$, $-\mathrm{C}=\mathrm{CH}$ vinylic); 7.58-7.67 (m, 5H, $\mathrm{C}^{3,5}-\overline{\mathrm{H}} \mathrm{s}$ of $\mathrm{p}$-Cl-phenyl ring, $\mathrm{C}^{3,5}-\underline{\mathrm{H}}$ s of pyrazole $\mathrm{N}^{1}$-phenyl, benzimidazole $\left.\mathrm{C}^{4}-\underline{\mathrm{H}}\right) ; \overline{7} .68-7.76\left(\mathrm{~m}, 3 \mathrm{H}, \mathrm{C}^{2,6}-\underline{\mathrm{Hs}}\right.$ of pyrazole $\mathrm{N}^{1}$ - $\underline{\text { phenyl }}$ and benzimidazole $\left.\mathrm{C}^{7}-\underline{\mathrm{H}}\right) ; 8.04\left(\mathrm{~d}, \mathrm{~J}=8 \mathrm{~Hz}, 2 \mathrm{H}, \mathrm{C}^{2,6}-\right.$ 
Hs of p-Cl-phenyl ring); 8.81 (s, $1 \mathrm{H}$, pyrazole $\left.\mathrm{C}^{5}-\mathrm{H}\right) ; 12.52$ (s, $1 \mathrm{H},-\mathrm{NH}$ of benzimidazole, $\mathrm{D}_{2} \mathrm{O}$ exchangeable). Anal. Calcd. for $\mathrm{C}_{34} \mathrm{H}_{30} \mathrm{ClN}_{7} \mathrm{OS}$ : C, 65.85; H, 4.88; N, 15.81. Found: $\overline{\mathrm{C}}$, 65.98; H, 4.96; N, 16.02 .

2-[1-(1H-Benzimidazole-2-yl)ethylidenehydrazono]-5-\{[3-(4-methoxyphenyl)-1-phenyl-1H-pyrazol-4-yl]methylene $\}-$ 3-cyclohexylthiazolidin-4-one

$5 x$

Yellow solid, yield 68\%, m.p. 215-7. FT-IR $\left(\mathrm{cm}^{-1}\right): 3337(\mathrm{~N}-\mathrm{H}) ; 1720(\mathrm{C}=\mathrm{O}) ; 1596(\mathrm{C}=\mathrm{N}) .{ }^{1} \mathrm{H}-\mathrm{NMR}$ (DMSO-d $\mathrm{d}_{6}$, Mercury $300 \mathrm{MHz}$ ): $\delta 1.11-1.47$ and 1.64-1.88 (2m, 10H, cyclohexyl C $\left.\mathrm{C}^{2,3,4,5,6}-\mathrm{H}_{2}\right) ; 2.60(\mathrm{~s}, 3 \mathrm{H}$, $\left.-\mathrm{N}=\mathrm{C}-\mathrm{CH}_{3}\right) ; 3.82\left(\mathrm{~s}, 3 \mathrm{H}, \mathrm{O}-\mathrm{CH}_{3}\right) ; 4.44-4.52\left(\mathrm{~m}, 1 \mathrm{H}\right.$, cyclohexyl $\left.\mathrm{C}^{1}-\mathrm{H}\right) ; 7.12\left(\mathrm{~d}, \mathrm{~J}=7.5 \overline{\mathrm{Hz}}, 2 \mathrm{H}, \mathrm{C}^{3,5}-\mathrm{Hs}\right.$ of p-OCH $-\mathrm{OCH}_{3}$-phenyl ring); 7.19-7.26 (dist. dd, $1 \mathrm{H}$, benzimidazole $\mathrm{C}^{5}-\mathrm{H}$ ); $7.28-7.35$ (dist. dd, $1 \mathrm{H}$, benzimidazole $\left.\mathrm{C}^{6}-\mathrm{H}\right) ; 7.40-7.48\left(\mathrm{~m}, 1 \mathrm{H}, \mathrm{C}^{4}-\mathrm{H}\right.$ of pyrazole $\mathrm{N}^{1}$-phenyl); $7.51(\mathrm{~s}, 1 \overline{\mathrm{H}},-\mathrm{C}=\mathrm{CH}$ vinylic); 7.56-7.64 (m, 5H, benzimidazole $\mathrm{C}^{4}-\mathrm{H}, \mathrm{C}^{2,3,5,6}-\mathrm{Hs}$ of pyrazole $\mathrm{N}^{1}$-phenyl); 7.68-7.74 (dist. $\mathrm{d}, 1 \overline{\mathrm{H}}$, benzimidazole $\mathrm{C}^{7}-\mathrm{H}$ ); 8.02 $\left(\mathrm{d}, \mathrm{J}=7.5 \mathrm{~Hz}, 2 \overline{\mathrm{H}}, \mathrm{C}^{2,6}{ }_{-} \mathrm{H}\right.$ s of $\mathrm{p}-\mathrm{OCH}_{3}$ phenyl ring); $8.77\left(\mathrm{~s}, 1 \mathrm{H}\right.$, pyrazole $\left.\mathrm{C}^{5}-\mathrm{H}\right) ; 12.56(\mathrm{~s}, 1 \mathrm{H},-\mathrm{NH}$ of benzimidazole, $\mathrm{D}_{2} \mathrm{O}$ exchangeable). Anal. Calcd. for $\mathrm{C}_{35} \mathrm{H}_{33} \mathrm{~N}_{7} \mathrm{O}_{2} \mathrm{~S} .1 / 2 \mathrm{H}_{2} \mathrm{O}$ : C, 67.29; H, 5.49; N, 15.69; $\mathrm{S}$, 5.13. Found: C, 66.89; H, 5.25; N, 15.50; S, 4.94.

General procedure for the synthesis of compounds $6 a-h$

To a mixture of 2-[1-(benzofuran-2-yl)ethylidenehydrazono]-3-substituted thiazolidin-4-ones or 2-[1-(1Hbenzimidazol-2-yl)ethylidenehydrazono]-3-substituted thiazolidin-4-ones $(\mathbf{3 a - i})(1 \mathrm{mmol})$ and isatin $(0.16 \mathrm{~g}$, $1.1 \mathrm{mmol})$ in dry dioxane $(5 \mathrm{ml})$, two drops of piperidine were added. The reaction mixture was refluxed for 4-6 h, concentrated, then allowed to cool to room temperature. The red precipitate formed after addition of ethanol $(10 \mathrm{ml})$ was filtered, washed with ethanol and recrystallized from dioxane/ethanol.

\section{2-[1-(Benzofuran-2-yl)ethylidenehydrazono]-5-(2-oxoindolin-3-ylidene)-3-phenylthiazolidin-4-one 6a}

Red solid, yield 63\%, m.p. >300. FT-IR $\left(\mathrm{cm}^{-1}\right): 3193(\mathrm{~N}-\mathrm{H}) ; 1705$ (C = O of thiazolidinone); 1687 (amide I band of isatin); $1606(\mathrm{C}=\mathrm{N}) ; 1263,1174,1082(\mathrm{C}-\mathrm{O}-\mathrm{C}) .{ }^{1} \mathrm{H}-\mathrm{NMR}\left(\mathrm{DMSO}-\mathrm{d}_{6}\right.$, Mercury $\left.300 \mathrm{MHz}\right): \delta 2.24$ $\left(\mathrm{s}, 3 \mathrm{H},-\mathrm{N}=\mathrm{C}^{-\mathrm{CH}_{3}}\right) ; 6.96\left(\mathrm{~d}, \mathrm{~J}=8 \mathrm{~Hz}, 1 \mathrm{H}\right.$, isatin $\left.\mathrm{C}^{7}-\mathrm{H}\right) ; 6.99-7.07$ (dist. dd, $1 \mathrm{H}$, isatin $\left.\mathrm{C}^{5}-\mathrm{H}\right)$; 7.27-7.7.61 ( $\mathrm{m}, 9 \mathrm{H}$, benzofuran $\mathrm{C}^{3,5,6}-\mathrm{Hs}$, Ar- $\mathrm{C}^{2,3,4,5,6}-\mathrm{Hs}$ and isatin $\left.\mathrm{C}^{6}-\mathrm{H}\right) ; 7.72-7.77\left(\mathrm{~m}, 2 \mathrm{H}\right.$, benzofuran $\left.\mathrm{C}^{4,7}-\mathrm{Hs}\right) ; 8.79$ $\left(\mathrm{d}, \mathrm{J}=8 \mathrm{~Hz}, 1 \mathrm{H}\right.$, isatin $\left.\mathrm{C}^{4}-\overline{\mathrm{H}}\right) ; 11.21\left(\mathrm{~s}, 1 \overline{\mathrm{H}},-\mathrm{NH}\right.$ of isatin, $\overline{\mathrm{D}_{2} \mathrm{O}}$ exchangeable). Anal. Calcd. for $\mathrm{C}_{27} \mathrm{H}_{18} \mathrm{~N}_{4} \mathrm{O}_{3} \mathrm{~S}$ : C, 67.77; H, 3.79; N, 11.71. Found: C, 67.91; $\overline{\mathrm{H}}, 3.84 ; \mathrm{N}, 11.87$.

\section{2-[1-(Benzofuran-2-yl)ethylidenehydrazono]-5-(2-oxoindolin-3-ylidene)-3-(4-chlorophenyl)thiazolidin-4-one 6b}

Red solid, yield 65\%, m.p. >300. FT-IR $\left(\mathrm{cm}^{-1}\right): 3189(\mathrm{~N}-\mathrm{H}) ; 1703(\mathrm{C}=\mathrm{O}$ of thiazolidinone); 1686 (amide I band of isatin); $1602(\mathrm{C}=\mathrm{N}) ; 1267,1170,1087(\mathrm{C}-\mathrm{O}-\mathrm{C}) .{ }^{1} \mathrm{H}-\mathrm{NMR}\left(\mathrm{DMSO}-\mathrm{d}_{6}\right.$, Mercury $\left.300 \mathrm{MHz}\right): \delta$ $2.26\left(\mathrm{~s}, 3 \mathrm{H},-\mathrm{N}=\mathrm{C}-\mathrm{CH}_{3}\right) ; 6.96\left(\mathrm{~d}, \mathrm{~J}=7.5 \mathrm{~Hz}, 1 \mathrm{H}\right.$, isatin $\left.\mathrm{C}^{7}-\underline{\mathrm{H}}\right) ; 6.99-7.08$ (dist. dd, $1 \mathrm{H}$, isatin $\left.\mathrm{C}^{5}-\underline{\mathrm{H}}\right)$; 7.27-7.48 (m, 3H, benzofuran $\mathrm{C}^{5,6}-\mathrm{Hs}$ and isatin $\left.\mathrm{C}^{6}-\mathrm{H}\right) ; 7.56\left(\mathrm{~s}, \overline{\mathrm{1}} \mathrm{H}\right.$, benzofuran $\left.\mathrm{C}^{3}-\mathrm{H}\right) ; 7.62-7.70(\mathrm{~m}, \overline{4 \mathrm{H}}$, Ar- $\left.\mathrm{C}^{2,3,5,6}-\mathrm{Hs}\right) ; 7.71-7.78\left(\mathrm{~m}, 2 \mathrm{H}\right.$, benzofuran $\left.\mathrm{C}^{4,7}-\mathrm{Hs}\right) ; 11.22$ (s, $1 \mathrm{H}$, $-\mathrm{NH}$ of isatin, $\mathrm{D}_{2} \mathrm{O}$ exchangeable). Anal. Calcd. for $\overline{\mathrm{C}}_{27} \mathrm{H}_{17} \mathrm{ClN}_{4} \mathrm{O}_{3} \mathrm{~S}$ : C, 63.22; H, 3.34; N, $\overline{10}$.92. Found: C, 63.34; $\mathrm{H}, 3.39 ; \mathrm{N}, 11.13$.

2-[1-(Benzofuran-2-yl)ethylidenehydrazono]-5-(2-oxoindolin-3-ylidene)-3-(4-methoxyphenyl)thiazolidin-4-one 6c

Red solid, yield 78\%, m.p. >300. FT-IR $\left(\mathrm{cm}^{-1}\right): 3180(\mathrm{~N}-\mathrm{H}) ; 1700(\mathrm{C}=\mathrm{O}$ of thiazolidinone); 1683 (amide I band of isatin); $1599(\mathrm{C}=\mathrm{N}) ; 1277,1167,1082(\mathrm{C}-\mathrm{O}-\mathrm{C}) .{ }^{1} \mathrm{H}-\mathrm{NMR}$ (DMSO-d ${ }_{6}$, Mercury $\left.300 \mathrm{MHz}\right): \delta 2.26$ $\left(\mathrm{s}, 3 \mathrm{H},-\mathrm{N}=\mathrm{C}_{-} \mathrm{CH}_{3}\right) ; 3.84\left(\mathrm{~s}, 3 \mathrm{H}, \mathrm{O}-\mathrm{CH}_{3}\right) ; 6.95\left(\mathrm{~d}, \mathrm{~J}=8 \mathrm{~Hz}, 1 \mathrm{H}\right.$, isatin $\left.\mathrm{C}^{7}-\mathrm{H}\right) ; 6.99-7.06($ dist. $\mathrm{dd}, 1 \mathrm{H}$, isatin $\left.\mathrm{C}^{5}-\mathrm{H}\right) ; 7.12\left(\mathrm{~d}, \mathrm{~J}=9 \mathrm{~Hz}, 2 \mathrm{H}, \mathrm{Ar}-\mathrm{C}^{3,5}-\overline{\mathrm{H}} \mathrm{s}\right) ; 7.28-7.45\left(\mathrm{~m}, 3 \mathrm{H}\right.$, isatin $\mathrm{C}^{6}-\mathrm{H}$ and benzofuran $\left.\mathrm{C}^{5,6}-\mathrm{Hs}\right) ; 7.50(\mathrm{~d}$, $\left.\mathrm{J}=9 \mathrm{~Hz}, 2 \mathrm{H}, \operatorname{Ar}-\mathrm{C}^{2,6}-\mathrm{Hs}\right) ; 7.55\left(\mathrm{~s}, 1 \overline{\mathrm{H}}\right.$, benzofuran $\left.\mathrm{C}^{3}-\mathrm{H}\right) ; 7.72-7.78\left(\overline{\mathrm{m}}, 2 \mathrm{H}\right.$, benzofuran $\left.\mathrm{C}^{4,7}-\overline{\mathrm{H}}\right) ; 8.80(\mathrm{~d}$, $\mathrm{J}=8 \mathrm{~Hz}, 1 \mathrm{H}$, isatin $\left.\mathrm{C}^{4}-\overline{\mathrm{H}}\right) ; 11.22\left(\mathrm{~s}, 1 \mathrm{H},-\mathrm{NH}\right.$ of isatin, $\overline{\mathrm{D}_{2} \mathrm{O}}$ exchangeable). Anal. Calcd. for $\mathrm{C}_{28} \mathrm{H}_{20} \mathrm{~N}_{4} \mathrm{O}_{4} \mathrm{~S}: \mathrm{C}$, 66.13; H, 3.96; N, 11.02; S, 6.31. Found: C, $65.77 ; \mathrm{H}, 3.85 ; \mathrm{N}, 11.01 ; \mathrm{S}, 6.68$.

2-[1-(Benzofuran-2-yl)ethylidenehydrazono]-5-(2-oxoindolin-3-ylidene)-3-cyclohexylthiazolidin-4-one 6d

Red solid, yield 73\%, m.p. > 300. FT-IR $\left(\mathrm{cm}^{-1}\right): 3166(\mathrm{~N}-\mathrm{H}) ; 1707$ (C = O of thiazolidinone); 1690 (amide I band of isatin); $1597(\mathrm{C}=\mathrm{N}) ; 1283,1175,1080(\mathrm{C}-\mathrm{O}-\mathrm{C}) .{ }^{1} \mathrm{H}-\mathrm{NMR}$ (DMSO-d ${ }_{6}$, Mercury $\left.300 \mathrm{MHz}\right): \delta 1.17-1.48$, 1.66-1.92 and 2.34-2.46 (3m, 10H, cyclohexyl $\left.\mathrm{C}^{2,3,4,5,6}-\underline{\mathrm{H}}_{2}\right) ; 2.51\left(\mathrm{~s}, 3 \mathrm{H},-\mathrm{N}=\mathrm{C}-\mathrm{CH}_{3}\right) ; 4.50-4.63(\mathrm{~m}, 1 \mathrm{H}$, cyclohexyl $\left.\mathrm{C}^{1}-\mathrm{H}\right) ; 6.94\left(\mathrm{~d}, \mathrm{~J}=7.7 \mathrm{~Hz}, 1 \mathrm{H}\right.$, isatin $\left.\mathrm{C}^{7}-\mathrm{H}\right) ; 7.02-7.10$ (dist. $\mathrm{dd}, 1 \mathrm{H}$, isatin $\left.\mathrm{C}^{5}-\mathrm{H}\right) ; 7.29-7.39(\mathrm{~m}$, $2 \mathrm{H}$, benzofuran $\left.\mathrm{C}^{5,6}-\underline{\mathrm{Hs}}\right) ; 7.40-7.48$ (dist. $\mathrm{dd}, 1 \mathrm{H}$, isatin $\left.\mathrm{C}^{6}-\underline{\mathrm{H}}\right) ; 7.60\left(\mathrm{~s}, 1 \mathrm{H}\right.$, benzofuran $\left.\mathrm{C}^{3}-\underline{\mathrm{H}}\right) ; 7.76(\mathrm{~m}, 2 \mathrm{H}$, 
benzofuran $\left.\mathrm{C}^{4,7}-\mathrm{Hs}\right) ; 8.87\left(\mathrm{~d}, \mathrm{~J}=7.7 \mathrm{~Hz}, 1 \mathrm{H}\right.$, isatin $\left.\mathrm{C}^{4}-\mathrm{H}\right) ; 11.15$ (s, 1H, -NH of isatin, $\mathrm{D}_{2} \mathrm{O}$ exchangeable). Anal. Calcd. for $\overline{\mathrm{C}}_{27} \mathrm{H}_{24} \mathrm{~N}_{4} \mathrm{O}_{3} \mathrm{~S}$ : C, 66.92; H, 4.99; N, 11.56. Found: C, 67.09; $\overline{\mathrm{H}}, 5.07 ; \mathrm{N}, 11.73$.

2-[1-(1H-Benzimidazole-2-yl)ethylidenehydrazono]-5-(2-oxoindolin-3-ylidene)-3-phenylthiazolidin-4-one 6e Red solid, yield 60\%, m.p. >300. FT-IR $\left(\mathrm{cm}^{-1}\right): 3257,3119(\mathrm{~N}-\mathrm{H}) ; 1704(\mathrm{C}=\mathrm{O}$ of thiazolidinone); 1680 (amide I band of isatin); $1607(\mathrm{C}=\mathrm{N}) .{ }^{1} \mathrm{H}-\mathrm{NMR}\left(\mathrm{DMSO}-\mathrm{d}_{6}\right.$, Mercury $\left.300 \mathrm{MHz}\right): \delta 2.33\left(\mathrm{~s}, 3 \mathrm{H},-\mathrm{N}=\mathrm{C}-\mathrm{CH}_{3}\right) ; 6.97$ $\left(\mathrm{d}, \mathrm{J}=7.5 \mathrm{~Hz}, 1 \mathrm{H}\right.$, isatin $\left.\mathrm{C}^{7}-\underline{\mathrm{H}}\right) ; 6.99-7.06$ (dist. dd, $1 \mathrm{H}$, isatin $\mathrm{C}^{5}-\underline{\mathrm{H}}$ ); 7.16-7.24 (dist. dd, 1H, benzimidazole $\left.\mathrm{C}^{5}-\underline{\mathrm{H}}\right) ; 7.26-7.40\left(\mathrm{~m}, 2 \mathrm{H}\right.$, benzimidazole $\mathrm{C}^{6}-\underline{\mathrm{H}}$ and isatin $\left.\mathrm{C}^{6}-\underline{\mathrm{H}}\right) ; 7 . \overline{5} 1-7.62\left(\mathrm{~m}, 6 \mathrm{H}\right.$, benzimidazole $\mathrm{C}^{4}-\underline{\mathrm{H}}$ and $\left.\operatorname{Ar}-\overline{\mathrm{C}^{2}}, 3,4,5,6-\underline{\mathrm{Hs}}\right) ; 7.69$ (d, J = $8 \mathrm{~Hz}, 1 \mathrm{H}$, benzimidazole $\left.\mathrm{C}^{7}-\underline{\mathrm{H}}\right) ; 8.78\left(\mathrm{~d}, \mathrm{~J}=7.5 \mathrm{~Hz}, 1 \mathrm{H}\right.$, isatin $\left.\mathrm{C}^{4}-\underline{\mathrm{H}}\right) ; \overline{11} .31$, $12.76\left(2 \mathrm{~s}\right.$, each $1 \mathrm{H},-\mathrm{NH}$ of isatin and benzimidazole, $\mathrm{D}_{2} \mathrm{O}$ exchangeable). Anal. Calcd. for $\mathrm{C}_{26} \mathrm{H}_{18} \overline{\mathrm{N}}_{6} \mathrm{O}_{2} \mathrm{~S}: \mathrm{C}_{\text {, }}$ 65.26; H, 3.79; N, 17.56. Found: C, 65.41; H, 3.85; N, 17.75.

\section{2-[1-(1H-Benzimidazole-2-yl)ethylidenehydrazono]-5-(2-oxoindolin-3-ylidene)-3-(4-chlorophenyl)thiazolidin-4-one $6 f$}

Red solid, yield 70\%, m.p. >300. FT-IR $\left(\mathrm{cm}^{-1}\right): 3260,3123(\mathrm{~N}-\mathrm{H}) ; 1714(\mathrm{C}=\mathrm{O}$ of thiazolidinone); 1682 (amide I band of isatin); $1610(\mathrm{C}=\mathrm{N}) .{ }^{1} \mathrm{H}-\mathrm{NMR}\left(\mathrm{DMSO}_{6} \mathrm{~d}_{6}\right.$, Mercury $\left.300 \mathrm{MHz}\right): \delta 2.35\left(\mathrm{~s}, 3 \mathrm{H},-\mathrm{N}=\mathrm{C}-\mathrm{CH}_{3}\right)$; $6.96\left(\mathrm{~d}, \mathrm{~J}=8 \mathrm{~Hz}, 1 \mathrm{H}\right.$, isatin $\left.\mathrm{C}^{7}-\mathrm{H}\right) ; 6.99-7.07$ (dist. $\mathrm{dd}, 1 \mathrm{H}$, isatin $\left.\mathrm{C}^{5}-\mathrm{H}\right) ; 7.16-7.40(\mathrm{~m}$, 3H, benzimidazole $\mathrm{C}^{5,6}-\mathrm{Hs}$ and isatin $\left.\mathrm{C}^{6}-\mathrm{H}\right) ; 7.58-7 . \overline{74}\left(\mathrm{~m}, 6 \mathrm{H}, \mathrm{Ar}-\mathrm{C}^{2,3,5,6}-\mathrm{Hs}\right.$ and benzimidazole $\left.\mathrm{C}^{4,7}-\mathrm{Hs}\right) ; 8.79(\mathrm{~d}, \mathrm{~J}=8 \mathrm{~Hz}, 1 \mathrm{H}$, isatin $\left.\mathrm{C}^{4}-\mathrm{H}\right) ; 11.23,1 \overline{2.72}\left(2 \mathrm{~s}\right.$, each $1 \mathrm{H},-\mathrm{NH}$ of isatin and benzimidazole, $\mathrm{D}_{2} \mathrm{O}$ exchangeable). EIMS m/z (\% abundance), $513(31.40) \mathrm{M}^{+}+1 ; 512(42 . \overline{72}) \mathrm{M}^{+}$; 64 (100). Anal. Calcd. for $\mathrm{C}_{26} \mathrm{H}_{17} \mathrm{ClN}_{6} \mathrm{O}_{2} \mathrm{~S}: \mathrm{C}, 60.88 ; \mathrm{H}$, 3.34; N, 16.38. Found: C, 60.83; H, 3.41; N, 16.49 .

\section{2-[1-(1H-Benzimidazole-2-yl)ethylidenehydrazono]-5-(2-oxoindolin-3-ylidene)-3-(4-methoxyphenyl)thiazolidin-4-one $6 g$}

Red solid, yield 65\%, m.p. >300. FT-IR $\left(\mathrm{cm}^{-1}\right): 3267,3133(\mathrm{~N}-\mathrm{H}) ; 1708(\mathrm{C}=\mathrm{O}$ of thiazolidinone); 1684 (amide I band of isatin); $1611(\mathrm{C}=\mathrm{N}) .{ }^{1} \mathrm{H}-\mathrm{NMR}\left(\mathrm{DMSO}_{\mathrm{d}}\right.$, Jeol $\left.500 \mathrm{MHz}\right): \delta 2.30\left(\mathrm{~s}, 3 \mathrm{H},-\mathrm{N}=\mathrm{C}_{-} \mathrm{CH}_{3}\right) ; 3.81(\mathrm{~s}$, $\left.3 \mathrm{H}, \mathrm{O}-\mathrm{CH}_{3}\right) ; 6.92\left(\mathrm{~d}, \mathrm{~J}=7.5 \mathrm{~Hz}, 1 \mathrm{H}\right.$, isatin $\left.\mathrm{C}^{7}-\underline{\mathrm{H}}\right) ; 6.98-7.04$ (dist. dd, $1 \mathrm{H}$, isatin $\left.\mathrm{C}^{5}-\mathrm{H}\right) ; 7.09 \overline{(\mathrm{d}}, \mathrm{J}=9 \mathrm{~Hz}$, $2 \mathrm{H}, \mathrm{Ar}-\mathrm{C}^{\overline{3,5}}-\underline{\mathrm{Hs}}$ ); 7.16-7.22 (dist. dd, $1 \mathrm{H}$, benzimidazole $\mathrm{C}^{5}-\underline{\mathrm{H}}$ ); $7.25-7.30$ (dist. dd, $1 \mathrm{H}$, benzimidazole $\mathrm{C}^{6}-\underline{\mathrm{H}}$ ); $7.30-7.36$ (dist. $\mathrm{dd}, 1 \mathrm{H}$, isatin $\mathrm{C}^{6}-\underline{\mathrm{H}}$ ); 7.47 (d, J = 9 Hz, 2H, $\overline{\mathrm{Ar}}-\mathrm{C}^{2,6}-\mathrm{Hs}$ ); 7.58 (d, J = $8 \mathrm{~Hz}, 1 \mathrm{H}$, benzimidazole $\left.\mathrm{C}^{4}-\underline{\mathrm{H}}\right) ; 7.66\left(\mathrm{~d}, \mathrm{~J}=8 \mathrm{~Hz}, 1 \mathrm{H}\right.$, benzimidazole $\left.\mathrm{C}^{7}-\underline{\mathrm{H}}\right) ; 8.78\left(\mathrm{~d}, \mathrm{~J}=7 . \overline{5} \mathrm{~Hz}, 1 \mathrm{H}\right.$, isatin $\left.\mathrm{C}^{4}-\underline{\mathrm{H}}\right) ; 11.24,12.73(2 \mathrm{~s}$, each $1 \mathrm{H},-\mathrm{NH}$ of isatin and benzimidazole, $\mathrm{D}_{2} \mathrm{O}$ exchangeable). Anal. Calcd. for $\mathrm{C}_{27} \mathrm{H}_{20} \overline{\mathrm{N}}_{6} \mathrm{O}_{3} \mathrm{~S}: \mathrm{C}, 63.77 ; \mathrm{H}$, 3.96 ; N, 16.53. Found: C, 63.85; H, 4.03; N, 16.71 .

\section{2-[1-(1H-Benzimidazole-2-yl)ethylidenehydrazono]-5-(2-oxoindolin-3-ylidene)-3-cyclohexylthiazolidin-4-one 6h}

Red solid, yield 56\%, m.p. > 300. FT-IR $\left(\mathrm{cm}^{-1}\right)$ : 3263, $3137(\mathrm{~N}-\mathrm{H}) ; 1715(\mathrm{C}=\mathrm{O}$ of thiazolidinone); 1680 (amide I band of isatin); $1602(\mathrm{C}=\mathrm{N}) .{ }^{1} \mathrm{H}-\mathrm{NMR}$ (DMSO-d 6 , Jeol $\left.500 \mathrm{MHz}\right): \delta$ 1.09-1.41, 1.60-1.90 and 2.29-2.42 (3m, $10 \mathrm{H}$, cyclohexyl $\left.\mathrm{C}^{2,3,4,5,6}-\underline{\mathrm{H}_{2}}\right) ; 2.58\left(\mathrm{~s}, 3 \mathrm{H},-\mathrm{N}=\mathrm{C}-\mathrm{CH}_{3}\right) ; 4.51-4.61\left(\mathrm{~m}, 1 \mathrm{H}\right.$, cyclohexyl $\left.\mathrm{C}^{1}-\underline{\mathrm{H}}\right) ; 6.91(\mathrm{~d}, \mathrm{~J}=8 \mathrm{~Hz}$, $1 \mathrm{H}$, isatin $\mathrm{C}^{7}-\underline{\mathrm{H}}$ ); $7.02-7.08$ (dist. dd, isatin $\mathrm{C}^{5}-\underline{\mathrm{H}}$ ); $\overline{7} .17-7.23$ (dist. dd, $1 \mathrm{H}$, benzimidazole $\mathrm{C}^{5}-\underline{\mathrm{H}}$ ); $7.25-7.31$ (dist. $\mathrm{dd}, 1 \mathrm{H}$, isatin $\left.\mathrm{C}^{6}-\underline{\mathrm{H}}\right) ; 7.31-7.37$ (dist. dd, $1 \overline{\mathrm{H}}$, benzimidazole $\mathrm{C}^{6}-\underline{\mathrm{H}}$ ); 7.59 (d, J = 8 Hz, 1H, benzimidazole $\left.\mathrm{C}^{4}-\mathrm{H}\right) ; 7.69\left(\mathrm{~d}, \mathrm{~J}=8 \mathrm{\textrm {Hz }}, 1 \mathrm{H}\right.$, benzimidazole $\left.\mathrm{C}^{7}-\underline{\mathrm{H}}\right) ; 8.86\left(\mathrm{~d}, \mathrm{~J}=8 \mathrm{~Hz}, 1 \mathrm{H}\right.$, isatin $\left.\mathrm{C}^{4}-\underline{\mathrm{H}}\right) ; 11.18$, $12.73(2 \mathrm{~s}$, each $1 \mathrm{H},-\mathrm{NH}$ of isatin and benzimidazole, $\mathrm{D}_{2} \mathrm{O}$ exchangeable). Anal. Calcd. for $\mathrm{C}_{26} \mathrm{H}_{24} \mathrm{~N}_{6} \mathrm{O}_{2} \mathrm{~S} .1 / 2 \mathrm{H}_{2} \mathrm{O}$ : C, 63.27; H, 5.11; N, 17.03; S, 6.50. Found: C, 63.23; H, 5.04; N, 16.67; S, 6.35.

\section{Synthesis of 2-\{[3-(4-chlorophenyl)-1-phenyl-1H-pyrazol-4-yl]methylenehydrazono\}-3-cyclohexyl-thiazolidin-4-one 7}

To a solution of 2-[1-(benzofuran-2-yl)ethylidenehydrazono]-3-cyclohexyl-thiazolidin-4-one or 2-[1-(1Hbenzimidazol-2-yl)ethylidenehydrazono]-3-cyclohexyl-thiazolidin-4-one (3b or 3i) (0.355 g, $1 \mathrm{mmol})$ and 3(4-chlorophenyl)-1-phenyl-1H-pyrazole-4-carbaldehyde (4b) $(0.31 \mathrm{~g}, 1.1 \mathrm{mmol})$ in glacial acetic acid $(5 \mathrm{ml})$, anhydrous sodium acetate $(0.13 \mathrm{~g}, 1.5 \mathrm{mmol})$ was added. The reaction mixture was refluxed for $10 \mathrm{~h}$, then filtered while hot and the solid separated was filtered, washed with ethanol and recrystallized from dioxane/ethanol.

Yellow solid, yield 65\%, m.p. >300. FT-IR $\left(\mathrm{cm}^{-1}\right): 1694(\mathrm{C}=\mathrm{O}) ; 1618(\mathrm{C}=\mathrm{N}) .{ }^{1} \mathrm{H}-\mathrm{NMR}\left(\mathrm{DMSO}-\mathrm{d}_{6}\right.$, Jeol $500 \mathrm{MHz}$ ): $\delta 1.11-1.27,1.55-1.78$ and 2.25-2.28 (3m, 10H, cyclohexyl $\left.\mathrm{C}^{2,3,4,5,6}-\mathrm{H}_{2}\right) ; 3.89$ (s, 2H, thiazolidinone $\left.\mathrm{C}^{5}-\underline{\mathrm{H}_{2}}\right)$; 4.20-4.32 (m, 1H, cyclohexyl $\left.\mathrm{C}^{1}-\mathrm{H}\right) ; 7.33-7.41\left(\mathrm{~m}, 1 \mathrm{H}, \mathrm{C}^{4}-\underline{\mathrm{H}}\right.$ of pyrazole $\mathrm{N}^{1}-$ phenyl); $7.48-7.63(\mathrm{~m}$, $4 \mathrm{H}, \overline{\mathrm{C}^{3}, 5}$-Hs of pyrazole $\mathrm{N}^{1}$-phenyl and $\mathrm{C}^{3,5}$ - $\mathrm{Hs}$ of $\mathrm{p}$-Cl-phenyl ring); $7 . \overline{91}$ (d, J = 7.5 Hz, 2H, $\mathrm{C}^{2}, 6$ - Hs of pyrazole

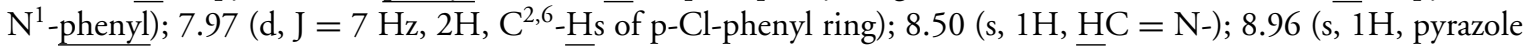


$\left.\mathrm{C}^{5}-\mathrm{H}\right)$. Anal. Calcd. for $\mathrm{C}_{25} \mathrm{H}_{24} \mathrm{ClN}_{5} \mathrm{OS} .1 / 2 \mathrm{H}_{2} \mathrm{O}$ : C, 61.65; H, 5.17; N, 14.38; S, 6.58. Found: C, 62.00; $\mathrm{H}$, $5.30 ; \mathrm{N}, 14.21 ; \mathrm{S}, 6.21$.

Synthesis of 3-cyclohexyl-5-(2-oxoindolin-3-ylidene)-2-[(2-oxoindolin-3-ylidene)hydrazono]thiazolidin-4-one 8

To a solution of 2-[1-(3-cyclohexyl-4-oxothiazolidin-2-ylidene)hydrazonoethyl]benzofuran (3d) $(0.355 \mathrm{~g}, 1 \mathrm{mmol})$ and isatin $(0.16 \mathrm{~g}, 1.1 \mathrm{mmol})$ in glacial acetic acid $(5 \mathrm{ml})$, anhydrous sodium acetate $(0.13 \mathrm{~g}, 1.5 \mathrm{mmol})$ was added. The reaction mixture was refluxed for $4 \mathrm{~h}$, then filtered while hot and the solid separated was filtered, washed with ethanol and recrystallized from dioxane/ethanol.

Red solid, yield 38\%, m.p. >300. FT-IR $\left(\mathrm{cm}^{-1}\right): 3160(\mathrm{~N}-\mathrm{H}) ; 1725$ (C = O of thiazolidinone); 1693 (amide I band of isatin); $1608(\mathrm{C}=\mathrm{N}) .{ }^{1} \mathrm{H}-\mathrm{NMR}\left(\mathrm{DMSO}_{\mathrm{d}}\right.$, Jeol $\left.500 \mathrm{MHz}\right): \delta 1.07-1.49$ and $1.56-1.94(2 \mathrm{~m}, 10 \mathrm{H}$, cyclohexyl $\left.\mathrm{C}^{2,3,4,5,6}-\mathrm{H}_{2}\right) ; 4.42-4.70\left(\mathrm{~m}, 1 \mathrm{H}\right.$, cyclohexyl $\left.\mathrm{C}^{1}-\mathrm{H}\right) ; 6.80-6.93\left(\mathrm{~m}, 2 \mathrm{H}\right.$, two isatin $\left.\mathrm{C}^{7}-\mathrm{Hs}\right) ; 6.96-7.08$ $\left(\mathrm{m}, 2 \mathrm{H}\right.$, two isatin $\left.\overline{\mathrm{C}^{5}-\mathrm{Hs}}\right) ; 7.27-7.41\left(\mathrm{~m}, 2 \mathrm{H}\right.$, two isatin $\left.\mathrm{C}^{6}-\mathrm{Hs}\right) ; 8.15$ and $8.80(2 \mathrm{~d}, \mathrm{~J}=7$ and $7 . \overline{5} \mathrm{~Hz}$, each $1 \mathrm{H}$, 2 isatin $\mathrm{C}^{4}-\mathrm{Hs}$ ); 10.79 and 11.18 ( 2 s, each $1 \mathrm{H}, 2-\mathrm{NHs}, \mathrm{D}_{2} \mathrm{O}$ exchangeable). Anal. Calcd. for $\mathrm{C}_{25} \mathrm{H}_{21} \mathrm{~N}_{5} \mathrm{O}_{3} \mathrm{~S}: \mathrm{C}$, 63.68; H, 4.49; N, 14.85. Found: C, 63.81; H, 4.54; N, 15.02 .

\section{Biological evaluation}

In vitro hemolytic assay

Blood samples $(5 \mathrm{ml})$ were freshly collected into heparinized tubes and were centrifuged (1000 r.p.m. for $20 \mathrm{~min}$ ) at room temperature. The plasma and buffy coat were removed by Pasteur pipette, and the collected erythrocytes were washed three-times using isotonic buffer solution ( $0.9 \%$ sodium chloride [Sigma-Aldrich, MO, USA]) by centrifugation (2500 r.p.m. for $10 \mathrm{~min}$ ). A two percent erythrocyte suspension was prepared to be used in the assay.

A $1 \mathrm{mg} / \mathrm{ml}$ stock solution of each compound was prepared in DMF (Sigma-Aldrich). Compound wells were prepared by adding $3 \mu \mathrm{l}$ of the compound solution to $117 \mu \mathrm{l}$ isotonic buffer solution and $120 \mu \mathrm{l}$ erythrocyte suspension. Compounds color blank wells were prepared by adding $3 \mu \mathrm{l}$ compound to $237 \mu \mathrm{l}$ isotonic buffer solution. Solvent (DMF) wells were prepared by adding $3 \mu \mathrm{l}$ DMF to $117 \mu$ isotonic buffer solution and $120 \mu \mathrm{l}$ erythrocyte suspension. Positive control wells (representing 100\% hemolysis) were prepared by adding $120 \mu \mathrm{l}$ erythrocyte suspension to $120 \mu \mathrm{l}$ distilled water, while negative control wells (representing $0 \%$ hemolysis) were prepared by adding $120 \mu \mathrm{l}$ erythrocyte suspension to $120 \mu \mathrm{l}$ isotonic buffer solution. Finally, blank wells contained only $240 \mu \mathrm{l}$ isotonic buffer solution. Each set of samples was pipetted in triplicate. The microtiter plate was then shaken well on bench, incubated at $37^{\circ} \mathrm{C}$ for $30 \mathrm{~min}$ then centrifuged ( $2000 \mathrm{rpm}$ for $5 \mathrm{~min}$ ) at room temperature.

A fraction of the supernatant layer $(150 \mu \mathrm{l})$ of each well was transferred to another 96 -well microtiter plate. Erythrocyte hemolysis was determined by reading the absorbance (A) of liberated hemoglobin at $405 \mathrm{~nm}$ in the supernatant fraction compared with the $100 \%$ hemolysis of erythrocyte. The average value was calculated from triplicate assay:

$$
\% \text { Hemolysis }=\frac{\left[\mathrm{A}_{\text {Sample }}-\left(\mathrm{A}_{\text {Color blank }}+\mathrm{A}_{\text {Solvent }}\right)\right]-\mathrm{A}_{\text {Saline }}}{\mathrm{A}_{100 \% \text { Hemolysis }}-\mathrm{A}_{\text {Saline }}} .
$$

It was decided during the present investigation that compounds which caused $<5 \%$ hemolysis were considered nontoxic to RBCs, while those which demonstrated $5 \%$ or more hemolysis were considered toxic and hemolytic on RBCs.

\section{In vitro anticancer screening}

HepG2 cells were routinely maintained as adherent cell cultures in RPMI-1640 medium (Lonza Group Ltd, Basel, Switzerland) supplemented with $10 \%$ fetal bovine serum (FBS; Lonza, IL, USA) at $37^{\circ} \mathrm{C}$ in a humidified air incubator containing $5 \% \mathrm{CO}_{2}$. Cells were subcultured for 2 weeks before assay. Cell viability was assessed using trypan blue exclusion method.

HepG2 cells were washed twice in RPMI-1640 medium supplemented with $200 \mu \mathrm{M}$ L-glutamine (Lonza) and $25 \mu \mathrm{M}$ 4-(2-hydroxyethyl)-1-piperazineethanesulfonic acid (HEPES) buffer (Lonza). The cells were suspended at $3 \times 10^{4}$ cells $/ \mathrm{ml}$ in RPMI culture medium (RPMI supplemented medium and $10 \%$ FBS). The appropriate number of cells was chosen to be $3 \times 10^{3}$ cells/well (100 $\mu$ l of the prepared suspension), and the cells were left to 
adhere on the polystyrene 96 -well plates in an incubator at $37^{\circ} \mathrm{C}, 5 \% \mathrm{CO}_{2}$ and $95 \%$ humidity for $24 \mathrm{~h}$. The cells were washed once using RPMI supplemented medium.

A $2 \mathrm{mg} / \mathrm{ml}$ stock solution of each compound was prepared in DMF and filtered using a $0.2 \mu \mathrm{M}$ syringe filter. The desired concentrations $(20,10,5,2.5$ and $1.25 \mu \mathrm{g} / \mathrm{ml})$ were prepared using serial dilution in a 96-well plate. Compound wells were prepared by adding $100 \mu \mathrm{l}$ of the previously prepared concentrations to a $100 \mu \mathrm{l}$ of HepG2 cells suspension. Parallel concentrations of the solvent were prepared to be used as controls. 5-Fluorouracil was used as a positive control. Control wells were prepared by adding $100 \mu \mathrm{l}$ culture media to a $100 \mu \mathrm{l}$ of HepG2 cells suspension. Blank wells contained $200 \mu$ l of culture media only (without cells or compound solution). Each set of samples was pipetted in duplicate. The plate was gently shaken, then incubated at $37^{\circ} \mathrm{C}, 5 \% \mathrm{CO}_{2}$ for $72 \mathrm{~h}$.

After incubation, the plate was centrifuged (2000 r.p.m. for $10 \mathrm{~min}$ ). The media were discarded by inversion over paper towels. A working solution $(100 \mu \mathrm{g} / \mathrm{ml})$ of neutral red stain (Bio Basic Inc, Markham, Canada) was prepared, and $100 \mu \mathrm{l}$ of this solution was added to each well, then the plate was gently shaken. The plate was incubated at $37^{\circ} \mathrm{C}$ in humidified $5 \% \mathrm{CO}_{2}$ for $3 \mathrm{~h}$, and then centrifuged (2000 r.p.m. for $10 \mathrm{~min}$ ). Excess dyes were discarded, and the cells were fixed with $100 \mu \mathrm{l}$ fixing solution $(0.5 \%$ formalin with $1 \%$ calcium chloride [Sigma-Aldrich]) for $1 \mathrm{~min}$. Cells were destained in $100 \mu \mathrm{l}$ destaining solution $(50 \%$ ethanol with $1 \%$ glacial acetic acid [Sigma-Aldrich]) for $5 \mathrm{~min}$ by shaking. The stain intensity was assayed using automated microplate reader spectrophotometer adjusted at $490 \mathrm{~nm}$. Surviving cell fraction was calculated according to the following equation:

$$
\text { Cell viability }(\%)=\frac{[\text { A of sample }+(\text { A of control }- \text { A of solvent })]-A \text { of blank }}{\text { A of control }- \text { A of blank }} \times 100
$$

The results were interpreted to calculate both the concentration causing 50\% cancer cell death $\left(\mathrm{IC}_{50}\right)$ of each compound and the maximum safe concentration that cause $100 \%$ viability $\left(\mathrm{LD}_{0}\right.$; to be used in the in vitro anti-HCV testing) using GraphPad InStat 3.0 software [47].

\section{In vitro cytotoxicity assay}

The PBMCs were isolated by density gradient centrifugation technique as described by Boyum [66]. Blood samples were freshly collected into heparinized or ethylenediaminetetraacetic acid sterile tubes. Blood was diluted using equal volume of RPMI-1640 medium containing 25 mM HEPES and then layered over equal volume of Ficoll-Pague ${ }^{\mathrm{TM}}$ Plus (Fisher BioReagents ${ }^{\text {TM }}$ Lymphocyte Separation Medium-LSM, PA, USA) and centrifuged (2000 r.p.m. for $30 \mathrm{~min}$ at acceleration and deceleration speeds zero \& zero) at room temperature. The buffy mononuclear cell layer was collected using sterile Pasteur pipette into $50 \mathrm{ml}$ sterile Falcon tube and washed twice in phosphatebuffered saline (Sigma-Aldrich) using centrifugation (1650 r.p.m. for 5 min). The isolated PBMCs viability was determined by hemocytometer count using the trypan blue exclusion method. The PBMCs were resuspended at $1 \times 10^{6}$ cells $/ \mathrm{ml}$ in RPMI-1640 medium containing 25 mM HEPES supplemented with $10 \%$ heat-inactivated FBS. The appropriate number of cells was chosen to be $1 \times 10^{5}$ cells/well (100 $\mu \mathrm{l}$ of the prepared suspension).

A $2 \mathrm{mg} / \mathrm{ml}$ stock solution of each compound was prepared in DMF and filtered using a $0.2 \mu \mathrm{M}$ syringe filter. The desired concentrations $(20,10,5,2.5$ and $1.25 \mu \mathrm{g} / \mathrm{ml})$ were prepared using serial dilution in a 96-well plate. Compound wells were prepared by adding $100 \mu \mathrm{l}$ of the previously prepared concentrations to a suspension of $1 \times 10^{5}$ PBMCs in $100 \mu \mathrm{l}$ culture media. Parallel concentrations of the solvent were prepared to be used as controls. Control wells were prepared by adding $100 \mu \mathrm{l}$ culture media to a suspension of $1 \times 10^{5}$ PBMCs in $100 \mu \mathrm{l}$ culture media. Blank wells contained $200 \mu \mathrm{l}$ of culture media only (without cells or compound solution). Each set of samples was pipetted in duplicate. The plate was then gently shaken then incubated at $37^{\circ} \mathrm{C}, 5 \% \mathrm{CO}_{2}$ for $72 \mathrm{~h}$.

After incubation, neutral red assay was performed as described previously, and surviving cell fraction was calculated according to the previously mentioned equation. Also, the results were interpreted to calculate both the lethal concentration that kills $50 \%$ of cells $\left(\mathrm{LD}_{50}\right)$ and the maximum safe concentration that cause $100 \%$ viability $\left(\mathrm{LD}_{0}\right)$ of each compound using GraphPad InStat 3.0 software [47].

\section{In vitro preliminary anti-HCV screening}

HepG2 cells were washed twice in RPMI-1640 medium supplemented with $200 \mu \mathrm{M} \mathrm{L}$-glutamine and $25 \mu \mathrm{M}$ HEPES buffer. The cells were suspended at $12 \times 10^{4}$ cells $/ \mathrm{ml}$ in RPMI culture medium (RPMI supplemented medium and $10 \%$ FBS). The appropriate number of cells to be used was chosen to be $12 \times 10^{3}$ cells $/$ well $(100 \mu \mathrm{l}$ 
of the prepared suspension), and the cells were left to adhere on the polystyrene 12-well plates in an incubator at $37^{\circ} \mathrm{C}, 5 \% \mathrm{CO}_{2}$ and $95 \%$ humidity for $24 \mathrm{~h}$. The cells were washed once using RPMI supplemented medium, then infected with $2 \% \mathrm{HCV}$-infected serum (the used serum was a pole of ten patients infected with HCV genotype-4 with viral loads: 3.2-9 million IU/ml, G4 HCV RNA) in RPMI culture medium containing 8\% FBS. The used dose of each compound was chosen so as to maintain 100\% viability of the HepG2 cell line (the dose was lower than the $\mathrm{LD}_{0}$ of each compound on HepG2 cells as calculated from the in vitro anticancer screening). It was reported that, cultured HepG2 cells lost her biotransformation activity due to decrease in cytochrome (CYP) transcription [67]. This waived its effect on the compounds stability during the test. Negative control is HepG2 cells with culture medium without addition of HCV infected serum or addition of tested compounds. Positive control is HepG2 cells with culture medium and infected HCV serum but without addition of tested compounds. After addition of the compounds, the plates were incubated at $37^{\circ} \mathrm{C}, 5 \% \mathrm{CO}_{2}$ and $95 \%$ humidity for $72 \mathrm{~h}$, followed by RNA extraction. The RNA strand was detected by RT-PCR using HCV-specific primers to the 59-untranslated region of the virus.

RNA was extracted from HepG2 cells using the method described by El-Awady et al. [50]. Culture cells were mixed with $200 \mu \mathrm{l}$ of $4 \mathrm{~mol} / \mathrm{l}$ guanidinium isothiocyanate (Fischer Bioreagents) containing $25 \mathrm{mmol} / \mathrm{l}$ sodium citrate (MP Biomedicals, CA, USA), 0.5\% sarkosyl (Fischer Bioreagents), $0.1 \mathrm{~mol} / 1 \beta$-mercaptoethanol (MP Biomedicals), and $100 \mu \mathrm{l}$ sodium acetate (Merck). The lysed cells were mixed with an equal volume of phenol, chloroform and isoamyl alcohol (25:24:1, $\mathrm{pH}$ 4; Fischer Bioreagents). After vortexing, the mixture was centrifuged $(14,000$ r.p.m. for $10 \mathrm{~min})$ at $4^{\circ} \mathrm{C}$. The aqueous layer was collected and mixed with an equal volume of isopropanol (Acros Organics, Geel, Belgium). After incubation at $-20^{\circ} \mathrm{C}$ overnight, RNA was precipitated by centrifugation (14,000 r.p.m. for $30 \mathrm{~min}$ ) at $4^{\circ} \mathrm{C}$, and the precipitated RNA was washed twice with $70 \%$ ethanol.

The complementary DNA and the first PCR reaction of the nested PCR detection system for the HCV-RNA were performed in a $50 \mu \mathrm{l}$ volume single-step reaction using the Ready-To-Go RT-PCR beads (GE Healthcare, Amersham, UK) and $10 \mu \mathrm{M}$ from each of the RT downstream primer $(1 \mathrm{CH})$, PCR forward primer $(2 \mathrm{CH})$ and reverse primer (P2) (Bioneer Corporation, Daejeon, Republic of Korea). The thermal cycling protocol was manipulated as follows: $30 \mathrm{~min}$ at $42^{\circ} \mathrm{C}$ for complementary DNA synthesis, followed by $5 \mathrm{~min}$ at $95^{\circ} \mathrm{C}, 35$ cycles of $1 \mathrm{~min}$ at $94^{\circ} \mathrm{C}, 1 \mathrm{~min}$ at $55^{\circ} \mathrm{C}, 1 \mathrm{~min}$ at $72^{\circ} \mathrm{C}$ and final extension $10 \mathrm{~min}$ at $72^{\circ} \mathrm{C}$.

The nested PCR amplification was performed in $50 \mu \mathrm{l}$ reaction mixture containing $0.2 \mathrm{mmol}$ from each dNTP (SibEnzyme Ltd., Novosibirsk, Russia), $10 \mu \mathrm{M}$ from each of the reverse nested primer (D2) and the forward nested primer (F2), two units of taq DNA polymerase (Thermo Fisher Scientific, MA USA) and $10 \mu$ from the RT-PCR reaction product in a $1 \times$ buffer. The thermal cycling protocol was manipulated as follows: $5 \mathrm{~min}$ at $95^{\circ} \mathrm{C}, 30$ cycles of $1 \mathrm{~min}$ at $95^{\circ} \mathrm{C}, 45 \mathrm{~s}$ at $58^{\circ} \mathrm{C}$ and $1 \mathrm{~min}$ at $72^{\circ} \mathrm{C}$ and final extension $10 \mathrm{~min}$ at $72^{\circ} \mathrm{C}$.

Primers' sequences were as follows; $1 \mathrm{CH}$ : 5'-ggtgcacggtctacgagacctc- $3^{\prime}, 2 \mathrm{CH}: 5^{\prime}$-aactactgtcttcacgcagaa- $3^{\prime}$, P2: $5^{\prime}$ tgctcatggtgcacggtcta-3', D2: 5'-actcggctagcagtctcgcg $3^{\prime}$ and F2: 5'-gtgcagcctccaggaccc-3'.

The PCR product was mixed with loading buffer by adding $10 \times$ bromophenol blue loading buffer to a final concentration of $1 \times$. DNA was separated by agarose gel electrophoresis using $2.5 \%$ agarose gel that was impregnated with ethidium bromide $(0.5 \mathrm{mg} / \mathrm{ml}$ [Sigma-Aldrich]) at $90 \mathrm{~V}$. The electrophoresis was run in standard TBE buffer $1 \times(54 \mathrm{~g}$ of Tris base, $27.5 \mathrm{~g}$ of boric acid and $20 \mathrm{ml}$ of $0.5 \mathrm{~mol} / \mathrm{l}$ ethylenediaminetetraacetic acid [pH 8] in $500 \mathrm{ml}$ of distilled water and a $1 \times$ solution is obtained by adding 1 part of the $10 \times$ TBE buffer to nine parts of distilled water). During electrophoresis DNA fragments were then visualized using a UV transilluminator, and a fragment of 174 base pairs length was identified in positive samples.

\section{Molecular modeling}

The molecular modeling studies were performed using the Molecular Operating Environment (MOE) [51] software [52]. The 3D structures and conformations of the enzymes were acquired from the PDB website [53].

The targeted compounds were drawn in MOE using the builder module, and collected in a database. The database was prepared by using the option 'Protonate 3D' to add hydrogens, calculate partial charges and minimize energy (using Force Field MMFF94x). In addition, the proteins were prepared by deleting the repeating chains, water molecules and any surfactants. Hydrogens were also added to the atoms of the receptor and the partial charges were calculated. The compounds' database was then docked into the pocket of each protein using the MOE dock. MOE was also used to calculate the best score between the ligands and the enzymes'-binding sites. Scoring was determined as a total of two specific scoring functions: London forces and affinity dG. The resulted database contained the score between the ligands' conformers and the enzymes'-binding sites in $\mathrm{kcal} / \mathrm{mol} .30$ 
conformers of each compound were retained with best score by default. The pose that showed the best score (lowest binding energy) was selected to show the ligand-enzyme interactions. To confirm the credibility of our docking results, the pose selection method was adopted to validate our docking protocol [68]. For all the used proteins, their co-crystallized ligands were drawn in MOE, prepared as the targeted compounds (hydrogens, partial charges and energy minimization) and then docked into the active site of the protein using our protocol. The conformer with the best score was superimposed on the original conformation and orientation of the co-crystallized ligand from the co-crystal structure acquired from the (PDB) using PyMOL software [69]. The root-mean-square deviation between the original and docked conformers was calculated by PyMOL and was $<1 \AA$ for all the ligands. It was reported that values $<1.5$ or $2 \AA$ were a sign of a successful and reliable docking protocol [68].

\section{Conclusion \& future perspective}

Molecular hybridization is an important concept in drug design based on the combination of pharmacophoric moieties of different bioactive substances to produce a new hybrid compound with improved affinity, selectivity and efficacy and reduced undesired side effects, when compared with the parent drugs. On the other hand, HCV is a contagious liver disease. The disease can be a mild illness lasting a few weeks or a serious, lifelong condition that can lead to liver cirrhosis which can progress to HCC. The HCV protein NS5A is activated by human kinases to produce the active phosphorylated form which upregulates COX-2 expression and promotes the release of matrix metalloprotinase- 2 and 9 associated with tumor progression and recurrence in HCC patients. Design and synthesis of hybrid molecules combining common pharmacophores for inhibiting both HCV and HCC will continue to be an objective for the development of new dual inhibitors of HCV and HCC with fewer side effects. In the current research, hybrid compounds were designed to incorporate both anti-HCV and anticancer pharmacophores. The anti-HCV pharmacophore was designed to include either benzofuran or benzimidazole scaffold linked to thiazolidinone moiety. The anticancer pharmacophore was planned to contain moieties like 1,3-diaryl-1H-pyrazole or 2-oxoindolin-3-ylidene able to inhibit kinases, specifically tyrosine kinases, to suppress HCC development, angiogenesis and potentiate the anti-HCV activity by inhibiting NS5A activation. Eight compounds exhibited potential in vitro anticancer activity against HCC cell line HepG2. Four compounds showed in vitro anti-HCV activity against HepG2 cells infected with HCV. As a result, compounds $\mathbf{5 l}$ and $\mathbf{5 p}$ were found to exhibit dual activity against $\mathrm{HCV}$ and HCC in vitro. Docking studies suggested that the newly synthesized compounds could suppress HCC through VEGFR2 tyrosine kinase inhibition.

\section{Author contribution}

MMM El-Miligy, SM Rida, FA Ashour, MH Badr and EM El-Bassiony were responsible for writing of the introduction and discussion, the rationale and experiments (chemistry and docking). MA El-Demellawy and AM Omar were responsible for biological screening (experimental procedures used and results).

\section{Summary points}

- Design and synthesize novel compounds that exhibit dual activity against hepatitis C virus (HCV) and its associated major complication, hepatocellular carcinoma (HCC) as an alternative to multidrug therapy.

- Hybrid compounds were designed to incorporate both anti-HCV and anticancer pharmacophores. The anti-HCV pharmacophore was designed to encompass different chemical scaffolds such as benzofuran, benzimidazole and thiazolidinone moieties. In addition, the anticancer pharmacophore was planned to contain moieties like 1,3-diaryl-1 H-pyrazole or 2-oxoindolin-3-ylidene able to inhibit kinases, specifically tyrosine kinases, to suppress HCC development, angiogenesis and potentiate the anti-HCV activity by inhibition of NS5A activation.

- Compounds $\mathbf{5 f}, \mathbf{5 j}, \mathbf{5 I}, \mathbf{5 p}, \mathbf{5 q}, \mathbf{5 r}, \mathbf{6 c}$ and $\mathbf{6 d}$ exhibited potential in vitro anticancer activity against $\mathrm{HCC}$ cell line HepG2.

- Compounds 5a, 5I, 5p and 5v showed in vitro anti-HCV activity against HepG2 cells infected with HCV.

- Compounds $5 \mathbf{5}$ and $\mathbf{5 p}$ were found to exhibit dual activity against HCV and HCC in vitro.

- Compound $\mathbf{5 q}$ showed a remarkable anticancer effect on $\mathrm{HepG} 2$ cancer cells with $\mathrm{IC}_{50}$ and IC 100 values $<100 \mu \mathrm{g} / \mathrm{ml}$ and less than its $\mathrm{LD}_{0}$ on peripheral blood mononuclear cell normal cells. This compound also showed a very high value of selectivity index indicating high selectivity toward cancerous HepG2 cells. This compound could be considered as a potent, safe and selective anticancer agent against HCC. This compound could be a lead one for further structure modification to achieve more potent anticancer agents.

- Docking studies suggested that the newly synthesized compounds could suppress HCC through VEGFR2 tyrosine kinase inhibition. 
Financial \& competing interests disclosure

This work was financially supported by funds from Alexandria University Research Fund (AURF); Research Enhancement Program (ALEX REP) as a part of the project entitled 'Design, synthesis and molecular modeling study of novel benzofurans and their bioisosteres as HCV and tumor inhibitors' (code: HLTH-10). MMM El-Miligy, SM Rida, FA Ashour, MH Badr and EM El-Bassiony are full-time employees of Pharmaceutical Chemistry Department, Faculty of Pharmacy, Alexandria University, Alexandria, Egypt. MA El-Demellawy and AM Omar are full-time employees of Genetic Engineering and Biotechnology Research Institute (GEBRI), City for Scientific Research and Technology, Alexandria, Egypt. The authors have no other relevant affiliations or financial involvement with any organization or entity with a financial interest in or financial conflict with the subject matter or materials discussed in the manuscript apart from those disclosed.

No writing assistance was utilized in the production of this manuscript.

Ethical conduct of research

The authors state that they have obtained appropriate institutional review board approval or have followed the principles outlined in the Declaration of Helsinki for all human or animal experimental investigations. In addition, for investigations involving human subjects, informed consent has been obtained from the participants involved.

\section{Open access}

This work is licensed under the Creative Commons Attribution 4.0 License. To view a copy of this license, visit http:// creativecommons.org/licenses/by/4.0/

\section{Supplementary data}

To view the supplementary data that accompany this paper please visit the journal website at: www.future-science.com/doi/suppl/ 10.4155/fsoa-2017-0075.

\section{References}

1. Stanaway JD, Flaxman AD, Naghavi M et al. The global burden of viral hepatitis from 1990 to 2013: findings from the Global Burden of Disease Study 2013. Lancet 388(10049), 1081-1088 (2016).

2. Hepatitis C. (2017). www.who.int/mediacentre/factsheets/fs164/en/

3. Manns MP, Buti M, Gane E et al. Hepatitis C virus infection. Nat. Rev. Dis. Primers 3, 17006 (2017).

4. Delemos AS, Chung RT. Hepatitis C treatment: an incipient therapeutic revolution. Trends Mol. Med. 20(6), 315-321 (2014).

5. Zhou C, Zhang W, Chen W et al. Integrated analysis of copy number variations and gene expression profiling in hepatocellular carcinoma. Sci. Rep. 7(1), 10570 (2017).

6. Herman P, Coelho FF. Laparoscopic resection for hepatocellular carcinoma: eastern and western experiences. Chin. J. Cancer Res. 26(3), 234-236 (2014).

7. Ghouri YA, Mian I, Rowe JH. Review of hepatocellular carcinoma: epidemiology, etiology, and carcinogenesis. J. Carcinog. 16, 1 (2017).

8. Yamauchi S, Takeuchi K, Chihara K et al. Hepatitis C virus particle assembly involves phosphorylation of NS5A by the c-Abl tyrosine kinase. J. Biol. Chem. 290(36), 21857-21864 (2015).

9. Nunez O, Fernandez-Martinez A, Majano PL et al. Increased intrahepatic cyclooxygenase 2, matrix metalloproteinase 2, and matrix metalloproteinase 9 expression is associated with progressive liver disease in chronic hepatitis $\mathrm{C}$ virus infection: role of viral core and NS5A proteins. Gut 53(11), 1665-1672 (2004).

10. Macdonald A, Harris M. Hepatitis C virus NS5A: tales of a promiscuous protein. J. Gen. Virol. 85(Pt 9), 2485-2502 (2004).

11. Kanda T, Yokosuka O, Omata M. Hepatitis C virus and hepatocellular carcinoma. Biology 2(1), 304-316 (2013).

12. Gale M, Jr, Kwieciszewski B, Dossett M, Nakao H, Katze MG. Antiapoptotic and oncogenic potentials of hepatitis $\mathrm{C}$ virus are linked to interferon resistance by viral repression of the PKR protein kinase. J. Virol. 73(8), 6506-6516 (1999).

13. De Mitri MS, Morsica G, Cassini R et al. Prevalence of wild-type in NS5A-PKR protein kinase binding domain in HCV-related hepatocellular carcinoma. J. Hepatol. 36(1), 116-122 (2002).

14. Huynh H. Molecularly targeted therapy in hepatocellular carcinoma. Biochem. Pharmacol. 80(5), 550-560 (2010).

15. Reig M, Torres F, Rodriguez-Lope C et al. Early dermatologic adverse events predict better outcome in HCC patients treated with sorafenib. J. Hepatol. 61(2), 318-324 (2014).

16. Koschny R, Gotthardt D, Koehler C, Jaeger D, Stremmel W, Ganten TM. Diarrhea is a positive outcome predictor for sorafenib treatment of advanced hepatocellular carcinoma. Oncology 84(1), 6-13 (2013).

17. Estfan B, Byrne M, Kim R. Sorafenib in advanced hepatocellular carcinoma: hypertension as a potential surrogate marker for efficacy. Am. J. Clin. Oncol. 36(4), 319-324 (2013). 
18. Gomaa A, Waked I. Management of advanced hepatocellular carcinoma: review of current and potential therapies. Practice 17, 18 (2017).

19. Kudo M. A new era of systemic therapy for hepatocellular carcinoma with regorafenib and lenvatinib. Liver Cancer 6(3), 177-184 (2017).

20. Sangro B, Iñarrairaegui M, Bilbao JI. Radioembolization for hepatocellular carcinoma. J. Hepatol. 56(2), 464-473 (2012).

21. Aerts M, Benteyn D, Van Vlierberghe H, Thielemans K, Reynaert H. Current status and perspectives of immune-based therapies for hepatocellular carcinoma. World J. Gastroenterol. 22(1), 253 (2016).

22. Kneteman NM, Howe AYM, Gao T et al. HCV796: a selective nonstructural protein 5B polymerase inhibitor with potent anti-hepatitis C virus activity In vitro, in mice with chimeric human livers, and in humans infected with hepatitis C virus. Hepatology 49(3), 745-752 (2009).

23. Chen YL, Zacharias J, Vince R, Geraghty RJ, Wang Z. C-6 aryl substituted 4-quinolone-3-carboxylic acids as inhibitors of hepatitis C virus. Bioorg. Med. Chem. 20(15), 4790-4800 (2012).

24. Vitale G, Corona P, Loriga M et al. 5-acetyl-2-arylbenzimidazoles as antiviral agents. Part 4. Eur. J. Med. Chem. 53, 83-97 (2012).

25. Kim ND, Chun H, Park SJ, Yang JW, Kim JW, Ahn SK. Discovery of novel HCV polymerase inhibitors using pharmacophore-based virtual screening. Bioorg. Med. Chem. Lett. 21(11), 3329-3334 (2011).

26. Kaushik-Basu N, Bopda-Waffo A, Talele TT, Basu A, Chen Y, Kucukguzel SG. 4-Thiazolidinones: a novel class of hepatitis C virus NS5B polymerase inhibitors. Front. Biosci. 13, 3857-3868 (2008).

27. Rawal RK, Katti SB, Kaushik-Basu N, Arora P, Pan Z. Non-nucleoside inhibitors of the hepatitis C virus NS5B RNA-dependant RNA polymerase: 2-Aryl-3-heteroaryl-1,3-thiazolidin-4-one derivatives. Bioorg. Med. Chem. Lett. 18(23), 6110-6114 (2008).

28. Kammasud N, Boonyarat C, Tsunoda S et al. Novel inhibitor for fibroblast growth factor receptor tyrosine kinase. Bioorg. Med. Chem. Lett. 17(17), 4812-4818 (2007).

29. Kammasud N, Boonyarat C, Sanphanya K et al. 5-Substituted pyrido[2,3-d]pyrimidine, an inhibitor against three receptor tyrosine kinases. Bioorg. Med. Chem. Lett. 19(3), 745-750 (2009).

30. Kim MH, Tsuhako AL, Co EW et al. The design, synthesis, and biological evaluation of potent receptor tyrosine kinase inhibitors. Bioorg. Med. Chem. Lett. 22(15), 4979-4985 (2012).

31. Liang G, Liu Z, Wu J, Cai Y, Li X. Anticancer molecules targeting fibroblast growth factor receptors. Trends Pharmacol. Sci. 33(10), 531-541 (2012).

32. Thaher BA, Arnsmann M, Totzke $\mathrm{F}$ et al. Tri- and tetrasubstituted pyrazole derivates: regioisomerism switches activity from $\mathrm{p} 38 \mathrm{MAP}$ kinase to important cancer kinases. J. Med. Chem. 55(2), 961-965 (2011).

33. Strocchi E, Fornari F, Minguzzi M et al. Design, synthesis and biological evaluation of pyrazole derivatives as potential multi-kinase inhibitors in hepatocellular carcinoma. Eur. J. Med. Chem. 48, 391-401 (2012).

34. Huang X-F, Lu X, Zhang Y et al. Synthesis, biological evaluation, and molecular docking studies of N-((1,3-diphenyl-1H-pyrazol-4-yl)methyl)aniline derivatives as novel anticancer agents. Biorg. Med. Chem. 20(16), 4895-4900 (2012).

35. Li X, Lu X, Xing M et al. Synthesis, biological evaluation, and molecular docking studies of N,1,3-triphenyl-1H-pyrazole-4-carboxamide derivatives as anticancer agents. Bioorg. Med. Chem. Lett. 22(11), 3589-3593 (2012).

36. Zhang J, Yang PL, Gray NS. Targeting cancer with small molecule kinase inhibitors. Nat. Rev. Cancer 9(1), 28-39 (2009).

37. Zheng $Y$, Zheng M, Ling X et al. Design, synthesis, quantum chemical studies and biological activity evaluation of pyrazole-benzimidazole derivatives as potent Aurora A/B kinase inhibitors. Bioorg. Med. Chem. Lett. 23(12), 3523-3530 (2013).

38. Miyamoto N, Sakai N, Hirayama T et al. Discovery of N-[5-(\{2-[(cyclopropylcarbonyl)amino]imidazo[1,2-b]pyridazin-6-yl $\}$ oxy)-2-methylph enyl]-1,3-dimethyl-1H-pyrazole-5-carboxamide (TAK-593), a highly potent VEGFR2 kinase inhibitor. Bioorg. Med. Chem. 21(8), 2333-2345 (2013).

39. Rida S, El-Hawash S, Fahmy H, Hazza A, El-Meligy M. Synthesis and in vitro evaluation of some novel benzofuran derivatives as potential anti-HIV-1, anticancer, and antimicrobial agents. Arch. Pharmacal Res. 29(1), 16-25 (2006).

40. Poyraz M, Sari M, Demirci F, Kosar M, Demirayak S, Büyükgüngör O. Synthesis, crystal structure and biological activity of 1-(1H-benzoimidazol-2-yl)-ethanone thiosemicarbazone and its cobalt complex. Polyhedron 27(9-10), 2091-2096 (2008).

41. Bondock S, Khalifa W, Fadda AA. Synthesis and antimicrobial evaluation of some new thiazole, thiazolidinone and thiazoline derivatives starting from 1-chloro-3,4-dihydronaphthalene-2-carboxaldehyde. Eur. J. Med. Chem. 42(7), 948-954 (2007).

42. Gawande SS, Warangkar SC, Bandgar BP, Khobragade CN. Synthesis of new heterocyclic hybrids based on pyrazole and thiazolidinone scaffolds as potent inhibitors of tyrosinase. Bioorg. Med. Chem. 21(10), 2772-2777 (2013).

43. Omar K, Geronikaki A, Zoumpoulakis P et al. Novel 4-thiazolidinone derivatives as potential antifungal and antibacterial drugs. Bioorg. Med. Chem. 18(1), 426-432 (2010).

44. Vicini P, Geronikaki A, Incerti M, Zani F, Dearden J, Hewitt M. 2-Heteroarylimino-5-benzylidene-4-thiazolidinones analogues of 2-thiazolylimino-5-benzylidene-4-thiazolidinones with antimicrobial activity: synthesis and structure-activity relationship. Bioorg. Med. Chem. 16(7), 3714-3724 (2008). 
45. Borenfreund E, Puerner J. A simple quantitative procedure using monolayer cultures for cytotoxicity assays (HTD/NR-90). J. Tissue Cult. Methods 9(1), 7-9 (1985).

46. Fotakis G, Timbrell JA. In vitro cytotoxicity assays: comparison of LDH, neutral red, MTT and protein assay in hepatoma cell lines following exposure to cadmium chloride. Toxicol. Lett. 160(2), 171-177 (2006).

47. GraphPad Software. www.graphpad.com/

48. Prayong P, Barusrux S, Weerapreeyakul N. Cytotoxic activity screening of some indigenous Thai plants. Fitoterapia 79(7-8), 598-601 (2008).

49. Kato N, Ikeda M, Mizutani T et al. Replication of hepatitis C virus in cultured non-neoplastic human hepatocytes. Jap. J. Cancer Res. 87(8), 787-792 (1996).

50. El-Awady MK, Ismail SM, El-Sagheer M, Sabour YA, Amr KS, Zaki EA. Assay for hepatitis C virus in peripheral blood mononuclear cells enhances sensitivity of diagnosis and monitoring of HCV-associated hepatitis. Clin. Chim. Acta 283(1-2), 1-14 (1999).

51. El-Nakkady SS, Roaiah HF, El-Serwy WS, Soliman AM, El-Moez SI, Abdel-Rahman AA. Antitumor and antimicrobial activities of some hetero aromatic benzofurans derived from naturally occurring visnagin. Acta Pol. Pharm. 69(4), 645-655 (2012).

52. Inc. CCG. Molecular Operating Environment (MOE) (2009). www.chemcomp.com/announcements/2009-12-08.htm

53. RCSB Protein Data Bank. www.rcsb.org/pdb/home/home.do

54. Howard S, Berdini V, Boulstridge JA et al. Fragment-based discovery of the pyrazol-4-yl urea (AT9283), a multitargeted kinase inhibitor with potent aurora kinase activity. J. Med. Chem. 52(2), 379-388 (2009).

55. Flint TJ, Hirschberg E, MurryMR. Comparative effects of chemotherapeutic agents on Brown-Pearce tumor and normal rabbit testis in vitro. Proc. Soc. Exp. Biol. Med. 84(2), 493-496 (1953).

56. Phillips MA. CCCXVII.:the formation of 2-substituted benziminazoles. J. Chem. Soc. 172, 2393-2399 (1928).

57. Ozegowski W, Krebs D. Aminosäureantagonisten. IV. Versuche zur Darstellung von [1-Methyl-5-bis-(B-chloräthyl)-amino-benzimidazolyl-(2)]-essigsäure. Journal für Praktische Chemie 29(1-2), 18-25 (1965).

58. Autenrieth W, Hinsberg O. Ueber einige Derivate des o-Toluylendiamins. Berichte der deutschen chemischen Gesellschaft 25(1), 604-607 (1892).

59. Georgescu M. Ueber einige Tetrahydroketochinoxaline. Berichte der deutschen chemischen Gesellschaft 25(1), 952-957 (1892).

60. Hinsberg O. Ueber Methylhydrooxytoluchinoxalin und die Constitution der aus $\alpha$-Oxysäuren und Orthodiaminen entstehenden Verbindungen. Berichte der deutschen chemischen Gesellschaft 25(2), 2416-2421 (1892).

61. Bistrzycki A, Przeworski G. Die Konstitution der Verbindungen aus o-Diaminen und $\alpha$-Oxysäuren. Acetylierung von Benzimidazolen. Berichte der deutschen chemischen Gesellschaft 45(3), 3483-3495 (1912).

62. Leng Z, Tao A, Xia Z, Gu X, Jin L, Wang R. Synthesis of the derivatives of 2-acetylbenzimidazolyl thiosemicarbazone. Zhongguo Yaoke Daxue Xuebao 21(5), 295-297 (1990).

63. Kira MA, Abdel-Rahman MO, Gadalla KZ. The vilsmeier-haack reaction - III Cyclization of hydrazones to pyrazoles. Tetrahedron Lett. 10(2), 109-110 (1969).

64. Bernard M, Hulley E, Molenda H, Stochla K, Wrzeciono U. [Azoles. 17. Beta-(4-pyrazol)acrylic and propionic acids and their anti-inflammatory activity]. Pharmazie 41(8), 560-562 (1986).

65. Prasath R, Bhavana P, Ng SW, Tiekink ER. 3-(4-Meth-oxy-phen-yl)-1-phenyl-1H-pyrazole-4-carbaldehyde. Acta Crystallogr. Sect. Sect. E: Struct. Rep. Online 67(Pt 11), o2962-o2963 (2011).

66. Boyum A. Isolation of mononuclear cells and granulocytes from human blood. Isolation of monuclear cells by one centrifugation, and of granulocytes by combining centrifugation and sedimentation at 1 g. Scand. J. Clin. Lab. Invest. Suppl. 97, 77-89 (1968).

67. Rodriguez-Antona C, Donato MT, Boobis A et al. Cytochrome P450 expression in human hepatocytes and hepatoma cell lines: molecular mechanisms that determine lower expression in cultured cells. Xenobiotica 32(6), 505-520 (2002).

68. Hevener KE, Zhao W, Ball DM et al. Validation of molecular docking programs for virtual screening against dihydropteroate synthase. $J$. Chem. Inf. Model. 49(2), 444-460 (2009).

69. Schrödinger L. PyMOL Molecular Graphics System Version 1.5.0.4. 